Supporting Information for:

\title{
Nickel-Catalyzed Intramolecular Hydroalkenylation of Imines
}

\author{
Wei-Min Feng, Tian-Yu Li, Li-Jun Xiao*, Qi-Lin Zhou* \\ State Key Laboratory and Institute of Elemento-Organic Chemistry, College of Chemistry, \\ Nankai University, Tianjin 300071, China \\ *Correspondence to: ljxiao@nankai.edu.cn (L.-J.X.); qlzhou@nankai.edu.cn (Q.-L.Z.)
}

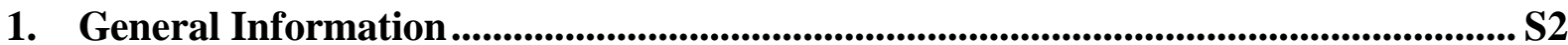

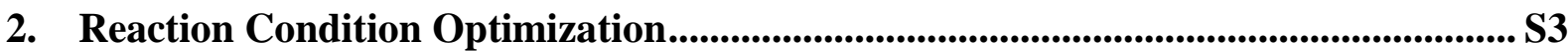

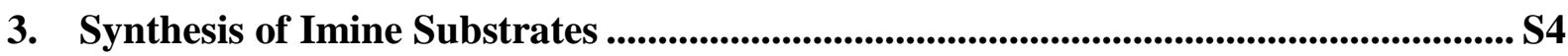

4. Nickel-Catalyzed Intramolecular Hydroalkenylation of Imines ................................ S14

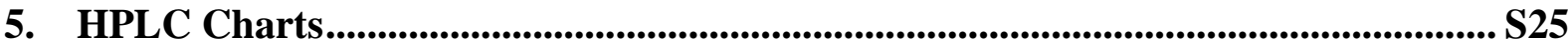

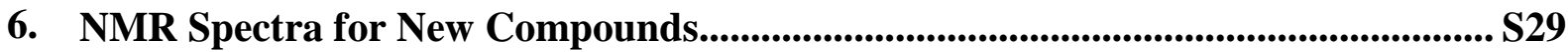

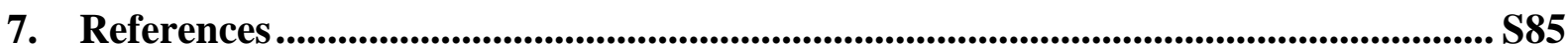




\section{General Information}

Methods: All reactions and manipulations which are sensitive to moisture or air were performed in an argon-filled glovebox (MBRAUN) or using standard Schlenk techniques. ${ }^{1} \mathrm{H}$ NMR and ${ }^{13} \mathrm{C}$ NMR spectra were recorded with a Brucker AV 400 spectrometer at $400 \mathrm{MHz}$ $\left({ }^{1} \mathrm{H}\right.$ NMR $), 101 \mathrm{MHz}\left({ }^{13} \mathrm{C}\right.$ NMR $), 162 \mathrm{MHz}\left({ }^{13} \mathrm{P}\right.$ NMR), and $376 \mathrm{MHz}\left({ }^{13} \mathrm{~F}\right.$ NMR $)$ in $\mathrm{CDCl}_{3}$. Chemical shifts were reported in ppm downfield from internal $\mathrm{Me}_{4} \mathrm{Si}$. HRMS were recorded on an IonSpec FT-ICR mass spectrometer with ESI resource or Q Exactive GC-Orbitrap MS with EI resource. Melting points were measured on a JIAHANG JH30 apparatus and uncorrected. Enantioselectivities were recorded on Agilent HPLC, using a chiral stationary phase column (Daicel Co. CHIRALPAK, CHIRALCEL, Phenomenex).

Materials: THF, toluene, and 1,4-dioxane were freshly dried and distilled from sodium using $\mathrm{Ph}_{2} \mathrm{CO}$ as the indicator. Hexane and DMA were freshly dried and distilled from $\mathrm{CaH}_{2}$. All chemicals reagents were purchased from Strem, Alfa Aeser, and Sigma-Aldrich, etc., and used without further purification. 


\section{Reaction Condition Optimization}

2.1 Screening of ligands ${ }^{a}$

\begin{tabular}{|c|c|c|c|c|}
\hline & ITs $^{\text {Th }}$ & $\begin{array}{c}\mathrm{Ni}(\mathrm{COD})_{2}(10 \mathrm{~mol} \%) \\
\text { Ligand }(12 \mathrm{~mol} \%) \\
\begin{array}{c}\mathrm{PhCOOH}(10 \mathrm{~mol} \%) \\
\mathrm{THF}, 90^{\circ} \mathrm{C}, 12 \mathrm{~h}\end{array}\end{array}$ & $\overbrace{}^{\mathrm{NHTS}}$ & $\underbrace{N T s}$ \\
\hline Entry & Ligand $^{a}$ & bite angle $\left({ }^{\circ}\right)^{b}$ & Yield of $2 \mathrm{a}(\%)$ & Yield of 3a (\%) \\
\hline 1 & $\mathbf{P P h}_{3}$ & - & 6 & 3 \\
\hline 2 & $\mathbf{P C y}_{3}$ & - & 6 & 3 \\
\hline 3 & $\mathbf{P}(n-\mathbf{B u})_{3}$ & - & 20 & $\mathbf{0}$ \\
\hline 4 & $\mathbf{P h}_{2} \mathbf{P E t}$ & - & 62 & 13 \\
\hline 5 & dppe & $87.3(86.5)$ & $<5$ & 3 \\
\hline 6 & dppp & $91.8(93)$ & 4 & 3 \\
\hline 7 & dppb & 96.1 (98.2) & 20 & 67 \\
\hline 8 & dppf & (104) & 16 & 70 \\
\hline 9 & DPPPent & $101.1(103.1)$ & 80 & 20 \\
\hline 10 & DPPhex & 111.4 & 75 & 15 \\
\hline 11 & Xantphos & 104.9 (109) & 49 & 46 \\
\hline 12 & DPEphos & $100.9(101.5)$ & 14 & 84 \\
\hline
\end{tabular}

${ }^{a} 22 \mathrm{~mol} \%$ for monophosphine ligand. ${ }^{b}$ Natural bite angles with nickel were calculated with PerkinElmer Chem3D, MM2; natural bite angles with palladium taken from ref 1 are given in parentheses.

\subsection{Screening of additives}

Additive $(10 \mathrm{~mol} \%)$
THF, $90^{\circ} \mathrm{C}, 12 \mathrm{~h}$

\subsection{Temperature evaluation}




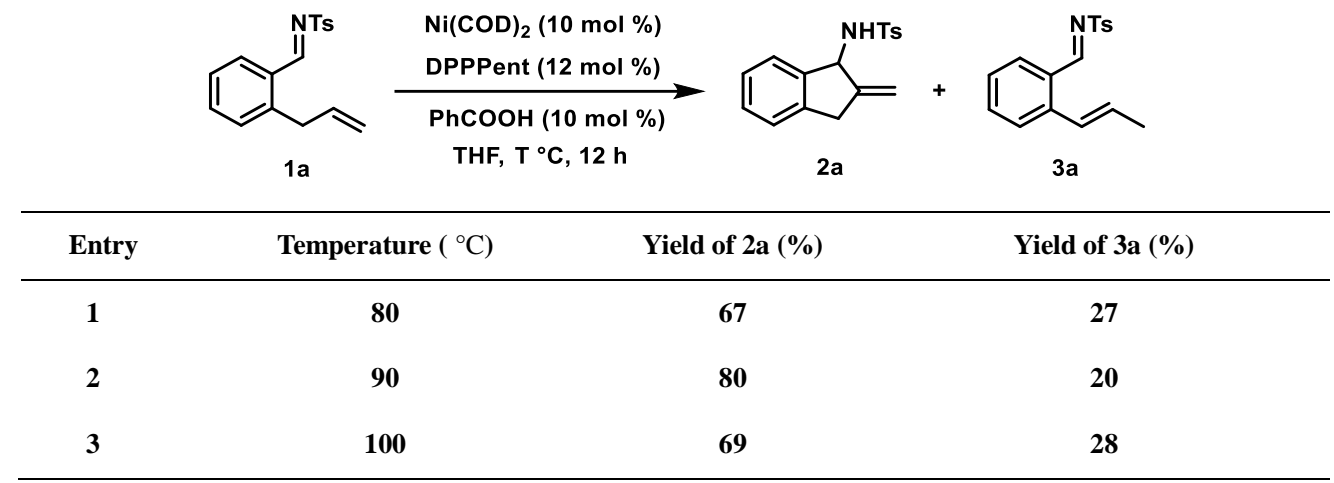

\subsection{Solvent evaluation}

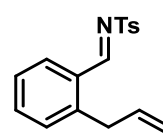

$1 \mathrm{a}$

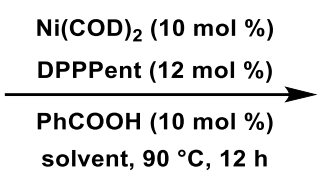

solvent, $90{ }^{\circ} \mathrm{C}, 12 \mathrm{~h}$

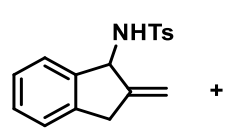

2a

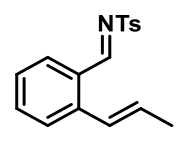

$3 \mathbf{a}$

\begin{tabular}{cccc}
\hline Entry & Solvent & Yield of 2a $(\%)$ & Yield of 3a $(\%)$ \\
\hline 1 & THF & 80 & 20 \\
2 & Hexane & 79 & 3 \\
3 & Toluene & 76 & 21 \\
4 & 1,4-Dioxane & 83 & 7 \\
5 & DMA & 79 & 17 \\
\hline
\end{tabular}

Yields were determined by ${ }^{1} \mathrm{H}$ NMR analysis using ethylene carbonate as internal standard.

\section{Synthesis of imine substrates}<smiles></smiles>

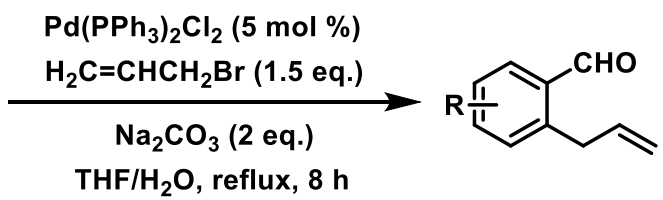

Procedure $\mathbf{A}^{2}$ : To a solution of the corresponding 2-formylphenylboronic acid (20 mmol, 1.0 equiv) and allyl bromide (30 mmol, 1.5 equiv) in THF $(60 \mathrm{~mL})$ in a round-bottom flask was added $\mathrm{PdCl}_{2}\left(\mathrm{PPh}_{3}\right)_{2}\left(0.5 \mathrm{mmol}, 0.025\right.$ equiv). The mixture was heated to $50{ }^{\circ} \mathrm{C}$ using heating module, then aq. $\mathrm{Na}_{2} \mathrm{CO}_{3}(1 \mathrm{M}, 2$ equiv) solution was added and heated at reflux for $4 \mathrm{~h}$. The reaction mixture was quenched with $\mathrm{H}_{2} \mathrm{O}$ and extracted with $\mathrm{CH}_{2} \mathrm{Cl}_{2}$. The combined organic layer was dried over $\mathrm{Na}_{2} \mathrm{SO}_{4}$, and concentrated in vacuum. The residue was purified by flash column chromatography on silica gel to afford the desired product.

Compound 1a'-1c', 1e', 1f', 1i', and 1j' were synthesized by procedure A. The analyses of new compounds are as follows. 


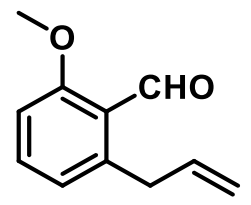

1b': petroleum ether/ethyl acetate $=20: 1(\mathrm{v} / \mathrm{v}) ;$ Yellow liquid, $1.8 \mathrm{~g}, 61 \%$ yield; ${ }^{1} \mathrm{H}$ NMR $(400$ $\left.\mathrm{MHz} \mathrm{CDCl}_{3}\right) \delta 10.58(\mathrm{~s}, 1 \mathrm{H}), 7.40(\mathrm{t}, J=8.0 \mathrm{~Hz}, 1 \mathrm{H}), 6.83(\mathrm{t}, J=7.5 \mathrm{~Hz}, 2 \mathrm{H}), 5.97$ (ddt, $J=$ $17.8,9.5,6.5 \mathrm{~Hz}, 1 \mathrm{H}), 5.05-4.96(\mathrm{~m}, 2 \mathrm{H}), 3.85(\mathrm{~s}, 3 \mathrm{H}), 3.72(\mathrm{dt}, J=6.7,1.6 \mathrm{~Hz}, 2 \mathrm{H}) .{ }^{13} \mathrm{C}$ $\operatorname{NMR}\left(101 \mathrm{MHz}, \mathrm{CDCl}_{3}\right) \delta 192.0,163.1,143.3,137.0,134.7,122.9,122.6,115.5,109.5,55.7$, 37.6. HRMS (EI) $m / z$ calcd for $\left[\mathrm{C}_{11} \mathrm{H}_{11} \mathrm{O}_{2}, \mathrm{M}-\mathrm{H}\right]^{+}$: 175.0754; found: 175.0754 .<smiles>C=CCc1cccc(F)c1C=O</smiles>

1c': petroleum ether/ethyl acetate $=20: 1(\mathrm{v} / \mathrm{v})$; Yellow liquid, $1.4 \mathrm{~g}, 49 \%$ yield; ${ }^{1} \mathrm{H}$ NMR $(400$ $\left.\mathrm{MHz}, \mathrm{CDCl}_{3}\right) \delta 10.52(\mathrm{~s}, 1 \mathrm{H}), 7.49(\mathrm{td}, J=8.0,5.7 \mathrm{~Hz}, 1 \mathrm{H}), 7.14-6.97$ (m, 2H), 5.99 (ddt, $J$ $=16.7,10.1,6.4 \mathrm{~Hz}, 1 \mathrm{H}), 5.12-4.96(\mathrm{~m}, 2 \mathrm{H}), 3.86-3.75(\mathrm{~m}, 2 \mathrm{H}) .{ }^{13} \mathrm{C} \mathrm{NMR}(101 \mathrm{MHz}$, $\left.\mathrm{CDCl}_{3}\right) \delta 189.1,189.0,167.4,164.9,144.1,136.3,135.4,135.3,126.7,126.7,122.1,122.0$, 116.4, 114.4, 114.2, 37.3, 37.3. ${ }^{19} \mathrm{~F}$ NMR $\left(376 \mathrm{MHz}, \mathrm{CDCl}_{3}\right) \delta-120.81$. HRMS (EI) $\mathrm{m} / z$ calcd for $\left[\mathrm{C}_{10} \mathrm{H}_{8} \mathrm{FO}, \mathrm{M}-\mathrm{H}\right]^{+}:$163.0554; found: 163.0553 .

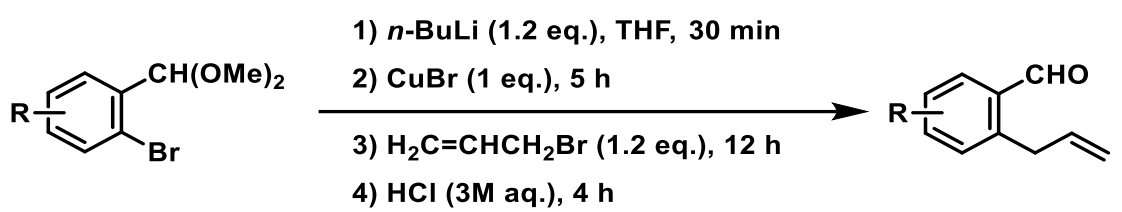

Procedure $\mathbf{B}^{3}$ : To a solution of substituted 1-bromo-2-(dimethoxymethyl)benzene (15 mmol, 1 equiv) in dry THF (30 mL) has added dropwise a solution of $n$-BuLi (2.4 M in hexane, 19.5 mmol, 1.3 equiv) at $-78^{\circ} \mathrm{C}$, and the mixture was stirred for $30 \mathrm{~min} . \mathrm{CuBr}$ (15 mmol, 1.0 equiv) was added portion-wise and the mixture was stirred at $-78{ }^{\circ} \mathrm{C}$ for $5 \mathrm{~h}$. To this mixture was added dropwise allyl bromide (18 mmol, 1.2 equiv), and the resultant mixture was allowed to warm to room temperature with stirring overnight. Then, $\mathrm{HCl}$ aq. (3 $\mathrm{M})$ was added to the mixture, and stirred for $3 \mathrm{~h}$. The organic layer was extracted with $\mathrm{CH}_{2} \mathrm{Cl}_{2}$ and dried over anhydrous $\mathrm{Na}_{2} \mathrm{SO}_{4}$. After the filtration, the solvent was removed under reduced pressure. The product was distilled in vacuum and used in the next step.

Compound 1g', 1h', 1k'-1p' were synthesized by procedure B. The analyses of new compounds are as follows. 


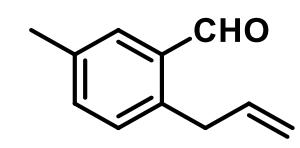

1g': Colorless liquid, $1.3 \mathrm{~g}, 56 \%$ yield; ${ }^{1} \mathrm{H}$ NMR (400 MHz, $\left.\mathrm{CDCl}_{3}\right) \delta 10.22(\mathrm{~s}, 1 \mathrm{H}), 7.66$ (s, 1H), $7.38-7.30(\mathrm{~m}, 1 \mathrm{H}), 7.18(\mathrm{~d}, J=7.7 \mathrm{~Hz}, 1 \mathrm{H}), 6.02$ (ddt, $J=16.5,10.1,6.1 \mathrm{~Hz}, 1 \mathrm{H}), 5.07$ $(\mathrm{dq}, J=10.1,1.5 \mathrm{~Hz}, 1 \mathrm{H}), 4.97(\mathrm{dq}, J=17.1,1.7 \mathrm{~Hz}, 1 \mathrm{H}), 3.77(\mathrm{dt}, J=6.1,1.7 \mathrm{~Hz}, 2 \mathrm{H}), 2.40$ (s, 3H). ${ }^{13} \mathrm{C} \mathrm{NMR}\left(101 \mathrm{MHz}, \mathrm{CDCl}_{3}\right) \delta 192.60,139.47,137.36,136.77,134.93,133.78,131.93$, 131.17, 116.30, 36.22, 20.93. HRMS (EI) $\mathrm{m} / z$ calcd for $\left[\mathrm{C}_{11} \mathrm{H}_{11} \mathrm{O}, \mathrm{M}-\mathrm{H}\right]^{+}$: 159.0804; found: 159.0805.

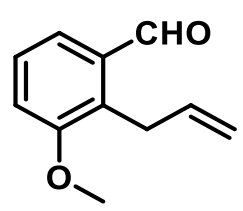

1l': Colorless liquid, $2.0 \mathrm{~g}, 77 \%$ yield; ${ }^{1} \mathrm{H}$ NMR (400 MHz, $\left.\mathrm{CDCl}_{3}\right) \delta 10.27(\mathrm{~s}, 1 \mathrm{H}), 7.51-7.45$ (m, 1H), $7.35(\mathrm{t}, J=8.0 \mathrm{~Hz}, 1 \mathrm{H}), 7.12(\mathrm{~d}, J=8.1,1 \mathrm{H}), 6.01(\mathrm{ddt}, J=17.1,10.0,5.9 \mathrm{~Hz}, 1 \mathrm{H})$, $5.01(\mathrm{dq}, J=10.2,1.6 \mathrm{~Hz}, 1 \mathrm{H}), 4.92(\mathrm{dq}, J=17.1,1.8 \mathrm{~Hz}, 1 \mathrm{H}), 3.89-3.82(\mathrm{~m}, 5 \mathrm{H}) .{ }^{13} \mathrm{C} \mathrm{NMR}$ $\left(101 \mathrm{MHz}, \mathrm{CDCl}_{3}\right) \delta 192.2,157.7,136.6,134.8,130.7,127.4,122.4,115.8,115.2,55.9,28.0$. HRMS (EI) $m / z$ calcd for $\left[\mathrm{C}_{11} \mathrm{H}_{11} \mathrm{O}_{2}, \mathrm{M}-\mathrm{H}\right]^{+}:$175.0754; found: 175.0754 .

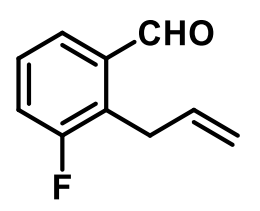

1m': Colorless liquid, $1.9 \mathrm{~g}, 78 \%$ yield; ${ }^{1} \mathrm{H}$ NMR (400 MHz, $\left.\mathrm{CDCl}_{3}\right) \delta 10.22$ (s, 1H), 7.67 (dd, $J=7.7,1.3 \mathrm{~Hz}, 1 \mathrm{H}), 7.39(\mathrm{td}, J=7.9,5.2 \mathrm{~Hz}, 1 \mathrm{H}), 7.34-7.27(\mathrm{~m}, 1 \mathrm{H}), 5.07(\mathrm{dq}, J=10.1,1.6$ $\mathrm{Hz}, 1 \mathrm{H}), 5.01-4.93(\mathrm{~m}, 1 \mathrm{H}), 3.84(\mathrm{dq}, J=5.7,1.7 \mathrm{~Hz}, 3 \mathrm{H}) .{ }^{13} \mathrm{C} \mathrm{NMR}\left(101 \mathrm{MHz}, \mathrm{CDCl}_{3}\right) \delta$ 191.1, 191.1, 162.4, 160.0, 135.7, 135.5, 128.9, 128.7, 128.0, 127.9, 127.2, 127.1, 120.8, 120.6, 116.1, 27.7, 27.6. ${ }^{19} \mathrm{~F}$ NMR (376 MHz, $\left.\mathrm{CDCl}_{3}\right) \delta-117.75$. HRMS (EI) $\mathrm{m} / z$ calcd for $\left[\mathrm{C}_{10} \mathrm{H}_{8} \mathrm{FO}\right.$, $\mathrm{M}-\mathrm{H}]^{+}:$163.0554; found: 163.0554 .

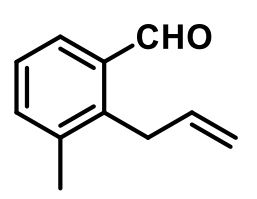

1n': Colorless liquid, $1.2 \mathrm{~g}, 76 \%$ yield; ${ }^{1} \mathrm{H}$ NMR (400 MHz, $\left.\mathrm{CDCl}_{3}\right) \delta 10.25$ (s, 1H), 7.75 $7.67(\mathrm{~m}, 1 \mathrm{H}), 7.44-7.38(\mathrm{~m}, 1 \mathrm{H}), 7.33-7.24(\mathrm{~m}, 1 \mathrm{H}), 6.00$ (ddt, $J=17.2,10.2,5.5 \mathrm{~Hz}, 1 \mathrm{H})$, $5.05(\mathrm{dq}, J=10.2,1.7 \mathrm{~Hz}, 1 \mathrm{H}), 4.83(\mathrm{dq}, J=17.1,1.9 \mathrm{~Hz}, 1 \mathrm{H}), 3.83(\mathrm{dt}, J=5.6,1.9 \mathrm{~Hz}, 2 \mathrm{H})$, 2.36 (s, 3H). ${ }^{13} \mathrm{C}$ NMR (101 MHz, $\left.\mathrm{CDCl}_{3}\right) \delta 192.9,140.2,138.4,136.0,136.0,134.4,129.4$, 126.7, 116.0, 31.8, 19.4. HRMS (EI) $\mathrm{m} / \mathrm{z}$ calcd for $\left[\mathrm{C}_{11} \mathrm{H}_{11} \mathrm{O}, \mathrm{M}-\mathrm{H}\right]^{+}$: 159.0804; found: 
159.0805 .
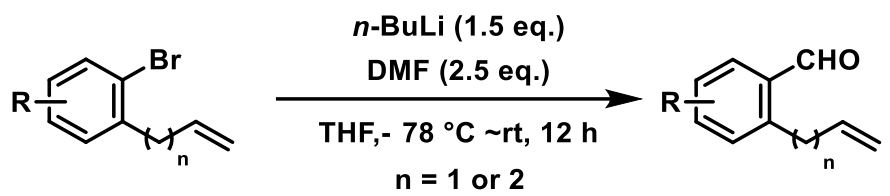

Procedure $\mathbf{C}^{3,4}$ : To a solution of 1-bromo-2-(but-3-en-1-yl)benzene (15 mmol, 1 equiv) in dry THF (30 mL) was added dropwise a solution of $n$-BuLi (2.4 M in hexane, $22.5 \mathrm{mmol}, 1.5$ equiv) at $-78{ }^{\circ} \mathrm{C}$, and the mixture was stirred for $30 \mathrm{~min}$. DMF (37.5 mmol, 2.5 equiv) was added and the resultant mixture was allowed to warm to room temperature with stirring overnight. The reaction mixture was quenched with $\mathrm{HCl}$ (aq. $3 \mathrm{M}$ ) and extracted with $\mathrm{CH}_{2} \mathrm{Cl}_{2}$. The combined organic layer was dried over $\mathrm{Na}_{2} \mathrm{SO}_{4}$, and concentrated in vacuum. The residue was distilled under reduced pressure to afford the desired product 1d'.

Compound 1d', $\mathbf{4 a} \mathbf{a}^{\prime}-\mathbf{4 f}$ ' was synthesized using procedure $C$. The analyses of new compounds are as follows.

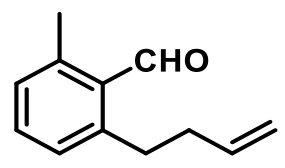

4d': Colorless liquid, $1.1 \mathrm{~g}, 67 \%$ yield, ${ }^{1} \mathrm{H}$ NMR $\left(400 \mathrm{MHz}, \mathrm{CDCl}_{3}\right) \delta 10.58(\mathrm{~s}, 1 \mathrm{H}), 7.36-$ $7.28(\mathrm{~m}, 1 \mathrm{H}), 7.11-7.05(\mathrm{~m}, 2 \mathrm{H}), 5.85$ (ddt, $J=16.9,10.1,6.6 \mathrm{~Hz}, 1 \mathrm{H}), 5.08-4.94(\mathrm{~m}, 2 \mathrm{H})$, $3.06-2.98(\mathrm{~m}, 2 \mathrm{H}), 2.59(\mathrm{~s}, 3 \mathrm{H}), 2.38-2.29(\mathrm{~m}, 2 \mathrm{H}) .{ }^{13} \mathrm{C} \mathrm{NMR}\left(101 \mathrm{MHz}, \mathrm{CDCl}_{3}\right) \delta 193.3$, 145.0, 141.1, 137.6, 132.9, 132.2, 130.0, 129.1, 115.3, 36.3, 32.8, 20.8. HRMS (EI) $\mathrm{m} / z$ calcd for $\left[\mathrm{C}_{12} \mathrm{H}_{13} \mathrm{O}, \mathrm{M}-\mathrm{H}\right]^{+}:$173.0961; found: 173.0959 .

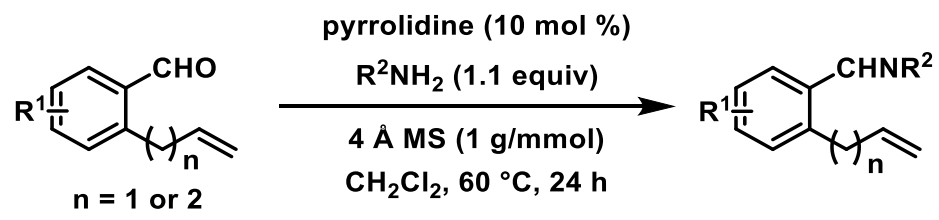

The imines were synthesized according to the literature method. ${ }^{5}$ The aldehyde $(5 \mathrm{mmol}, 1$ equiv), amine (5.5 mmol, 1.1. equiv), DCM (30 mL), and $4 \AA$ molecule sieve $(1 \mathrm{~g} / \mathrm{mmol})$ were added to a sealed tube. After the pyrrolidine $(0.5 \mathrm{mmol}, 0.1$ equiv) was added, the mixture was heated at $60{ }^{\circ} \mathrm{C}$ using oil bath for $24 \mathrm{~h}$, and was cooled to room temperature. The liquid was filtered out, and the residue was purified by flash chromatography (petro ether/ethyl acetate = 5:1). The analyses of new compounds are as follows. 
<smiles>C=CCc1cccc(OC)c1C=[S+](C)(C)C</smiles>

1b: petroleum ether/ethyl acetate $=5: 1(\mathrm{v} / \mathrm{v})$; Yellow solid, $902 \mathrm{mg}, 48 \%$ yield; m.p. $87-89{ }^{\circ} \mathrm{C}$; ${ }^{1} \mathrm{H} \mathrm{NMR}\left(400 \mathrm{MHz}, \mathrm{CDCl}_{3}\right) \delta 9.64(\mathrm{~s}, 1 \mathrm{H}), 7.90-7.84(\mathrm{~m}, 2 \mathrm{H}), 7.44(\mathrm{t}, J=8.1 \mathrm{~Hz}, 1 \mathrm{H}), 7.32$ $(\mathrm{d}, J=7.8 \mathrm{~Hz}, 2 \mathrm{H}), 6.89-6.81(\mathrm{~m}, 2 \mathrm{H}), 5.93-5.75(\mathrm{~m}, 1 \mathrm{H}), 4.93-4.86(\mathrm{~m}, 2 \mathrm{H}), 3.89(\mathrm{~s}, 3 \mathrm{H})$, $3.79(\mathrm{dt}, J=6.5,1.5 \mathrm{~Hz}, 2 \mathrm{H}), 2.43(\mathrm{~s}, 3 \mathrm{H}) .{ }^{13} \mathrm{C} \mathrm{NMR}\left(101 \mathrm{MHz}, \mathrm{CDCl}_{3}\right) \delta 167.7,162.8,145.3$, 144.2, 136.8, 136.1, 135.6, 129.8, 128.0, 123.6, 119.1, 116.0, 109.3, 56.1, 38.9, 21.8. HRMS (ESI) $\mathrm{m} / z$ calcd for $\left[\mathrm{C}_{18} \mathrm{H}_{19} \mathrm{NO}_{3} \mathrm{SNa}, \mathrm{M}+\mathrm{Na}\right]^{+}:$352.0978; found: 352.0981 .<smiles>C=CCc1cccc(F)c1C=[S+]=[N-]</smiles>

1c: petroleum ether/ethyl acetate $=5: 1(\mathrm{v} / \mathrm{v})$; Yellow solid, $400 \mathrm{mg}, 21 \%$ yield; m.p. $60-62{ }^{\circ} \mathrm{C}$; ${ }^{1} \mathrm{H}$ NMR $\left(400 \mathrm{MHz}, \mathrm{CDCl}_{3}\right) \delta 9.42(\mathrm{~s}, 1 \mathrm{H}), 7.91-7.85(\mathrm{~m}, 2 \mathrm{H}), 7.49(\mathrm{td}, J=8.0,5.7 \mathrm{~Hz}, 1 \mathrm{H})$, $7.35(\mathrm{~d}, J=8.0 \mathrm{~Hz}, 2 \mathrm{H}), 7.14-7.01(\mathrm{~m}, 2 \mathrm{H}), 5.85$ (ddt, $J=16.7,10.1,6.4 \mathrm{~Hz}, 1 \mathrm{H}), 5.01-4.88$ $(\mathrm{m}, 2 \mathrm{H}), 3.81(\mathrm{dt}, J=6.6,1.5 \mathrm{~Hz}, 2 \mathrm{H}), 2.45(\mathrm{~s}, 3 \mathrm{H}) .{ }^{13} \mathrm{C} \mathrm{NMR}\left(101 \mathrm{MHz}, \mathrm{CDCl}_{3}\right) \delta 166.4$, 164.8, 164.7, 163.8, 145.5, 144.8, 136.0, 135.8, 135.7, 135.1, 129.9, 128.2, 127.1, 127.1, 118.8, 118.7, 116.8, 114.4, 114.2, 38.3, 21.8. ${ }^{19} \mathrm{~F}$ NMR (376 MHz, $\mathrm{CDCl}_{3}$ ) $\delta-112.29$. HRMS (ESI) $m / z$ calcd for $\left[\mathrm{C}_{17} \mathrm{H}_{16} \mathrm{FNO}_{2} \mathrm{SNa}, \mathrm{M}+\mathrm{Na}\right]^{+}: 340.0778$; found: 340.0782 .<smiles>C=CCc1cccc(C)c1C=[S+]=[N-]</smiles>

1d: petroleum ether/ethyl acetate $=5: 1(\mathrm{v} / \mathrm{v})$; White solid, $213 \mathrm{mg}, 11 \%$ yield; m.p. $79-80{ }^{\circ} \mathrm{C}$; ${ }^{1} \mathrm{H}$ NMR $\left(400 \mathrm{MHz}, \mathrm{CDCl}_{3}\right) \delta 9.42(\mathrm{~s}, 1 \mathrm{H}), 7.87(\mathrm{~d}, J=8.3 \mathrm{~Hz}, 1 \mathrm{H}), 7.39-7.31(\mathrm{~m}, 3 \mathrm{H}), 7.17$ $-7.10(\mathrm{~m}, 2 \mathrm{H}), 5.88$ (ddt, $J=16.4,10.1,6.1 \mathrm{~Hz}, 1 \mathrm{H}), 5.01$ (dq, $J=10.1,1.6 \mathrm{~Hz}, 1 \mathrm{H}), 4.90$ (dq, $J=17.0,1.7 \mathrm{~Hz}, 1 \mathrm{H}), 3.66(\mathrm{dt}, J=6.1,1.7 \mathrm{~Hz}, 2 \mathrm{H}), 2.56(\mathrm{~s}, 3 \mathrm{H}), 2.44(\mathrm{~s}, 3 \mathrm{H}) .{ }^{13} \mathrm{C} \mathrm{NMR}(101$ $\left.\mathrm{MHz}, \mathrm{CDCl}_{3}\right) \delta 169.6,144.5,143.9,142.4,136.9,135.5,133.5,130.4,129.8,129.1,128.9$, 128.1, 116.6, 38.3, 22.1, 21.7. HRMS (ESI) $\mathrm{m} / \mathrm{z}$ calcd for $\left[\mathrm{C}_{18} \mathrm{H}_{20} \mathrm{NO}_{2} \mathrm{~S}, \mathrm{M}+\mathrm{H}\right]^{+}:$314.1209; found: 314.1210 .<smiles></smiles> 
1f: petroleum ether/ethyl acetate $=5: 1(\mathrm{v} / \mathrm{v})$; Yellow solid, $514 \mathrm{mg}, 54 \%$ yield; m.p. $82-84{ }^{\circ} \mathrm{C}$; ${ }^{1} \mathrm{H}$ NMR $\left(400 \mathrm{MHz}, \mathrm{CDCl}_{3}\right) \delta 9.25(\mathrm{~d}, J=2.1 \mathrm{~Hz}, 1 \mathrm{H}), 7.89-7.85(\mathrm{~m}, 2 \mathrm{H}), 7.78(\mathrm{dd}, J=9.2$, $2.7 \mathrm{~Hz}, 1 \mathrm{H}), 7.36(\mathrm{~d}, J=8.1 \mathrm{~Hz}, 2 \mathrm{H}), 7.29-7.20(\mathrm{~m}, 3 \mathrm{H}), 6.01-5.89(\mathrm{~m}, 1 \mathrm{H}), 5.14-5.08(\mathrm{~m}$, $1 \mathrm{H}), 4.94-4.87(\mathrm{~m}, 1 \mathrm{H}), 3.67-3.63(\mathrm{~m}, 2 \mathrm{H}), 2.45$ (s, 3H). ${ }^{13} \mathrm{C} \mathrm{NMR}\left(101 \mathrm{MHz}, \mathrm{CDCl}_{3}\right) \delta$ 167.1, 167.1, 162.8, 160.3, 144.9, 139.5, 139.4, 136.7, 134.9, 132.9, 132.8, 132.2, 132.1, 129.9, 128.3, 122.0, 121.8, 117.2, 115.8, 115.6, 36.3, 21.8. ${ }^{19} \mathrm{~F}$ NMR $\left(376 \mathrm{MHz}, \mathrm{CDCl}_{3}\right) \delta-114.3$. HRMS (ESI) $m / z$, calcd for $\left[\mathrm{C}_{17} \mathrm{H}_{16} \mathrm{FNO}_{2} \mathrm{SNa}, \mathrm{M}+\mathrm{Na}\right]^{+}: 340.0778$; found: 340.0783 .<smiles>C=CCc1ccc(C)cc1C=[N+]=[N-]</smiles>

1g: petroleum ether/ethyl acetate $=5: 1(\mathrm{v} / \mathrm{v})$; Yellow solid, $990 \mathrm{mg}, 81 \%$ yield; m.p. $75-77{ }^{\circ} \mathrm{C}$; ${ }^{1} \mathrm{H}$ NMR (400 MHz, $\left.\mathrm{CDCl}_{3}\right) \delta 9.27(\mathrm{~s}, 1 \mathrm{H}), 7.93$ - $7.83(\mathrm{~m}, 3 \mathrm{H}), 7.37$ - 7.31 (m, 3H), 7.17 (d, $J=7.8 \mathrm{~Hz}, 1 \mathrm{H}), 6.02-5.87(\mathrm{~m}, 1 \mathrm{H}), 5.11-5.01(\mathrm{~m}, 1 \mathrm{H}), 4.95-4.85(\mathrm{~m}, 1 \mathrm{H}), 3.65(\mathrm{~d}, J=5.9$ $\mathrm{Hz}, 2 \mathrm{H}), 2.44$ (s, 3H), 2.33 (s, 3H). ${ }^{13} \mathrm{C} \mathrm{NMR}\left(101 \mathrm{MHz}, \mathrm{CDCl}_{3}\right) \delta 168.7,144.5,140.9,137.1$, 137.0, 135.9, 135.3, 131.0, 130.3, 130.1, 129.8, 128.1, 116.8, 36.7, 21.7, 20.8. HRMS (ESI) Calcd for $\left[\mathrm{C}_{18} \mathrm{H}_{20} \mathrm{NO}_{2} \mathrm{~S}, \mathrm{M}+\mathrm{H}\right]^{+}:$314.1209; found: 314.1211 .<smiles>C=CCc1ccc(C(F)(F)F)cc1C=[N+]=[N-]</smiles>

1h: petroleum ether/ethyl acetate $=5: 1(\mathrm{v} / \mathrm{v})$; White solid; m.p. $102-104{ }^{\circ} \mathrm{C} ;{ }^{1} \mathrm{H}$ NMR $(400$ $\left.\mathrm{MHz}, \mathrm{CDCl}_{3}\right) \delta 9.32(\mathrm{~s}, 1 \mathrm{H}), 8.34(\mathrm{~s}, 1 \mathrm{H}), 7.88(\mathrm{~d}, J=8.3 \mathrm{~Hz}, 2 \mathrm{H}), 7.75(\mathrm{dd}, J=8.1,2.0 \mathrm{~Hz}$, $1 \mathrm{H}), 7.43(\mathrm{~d}, J=8.1 \mathrm{~Hz}, 1 \mathrm{H}), 7.37(\mathrm{~d}, J=8.2 \mathrm{~Hz}, 2 \mathrm{H}), 5.96$ (ddt, $J=16.5,10.2,6.0 \mathrm{~Hz}, 1 \mathrm{H})$, $5.19-5.11(\mathrm{~m}, 1 \mathrm{H}), 4.99-4.89(\mathrm{~m}, 1 \mathrm{H}), 3.79-3.70(\mathrm{~m}, 2 \mathrm{H}), 2.45(\mathrm{~s}, 3 \mathrm{H}) .{ }^{13} \mathrm{C}$ NMR $(101$ $\left.\mathrm{MHz}, \mathrm{CDCl}_{3}\right) \delta 166.9,147.1,145.1,135.8,134.7,131.8,131.1,130.8$ (q, $J_{\mathrm{C}, \mathrm{F}}=3.4 \mathrm{~Hz}$ ), 130.1, 128.4, $126.9\left(\mathrm{q}, J_{\mathrm{C}, \mathrm{F}}=3.7 \mathrm{~Hz}\right), 118.0,37.0,21.8 .{ }^{19} \mathrm{~F} \mathrm{NMR}\left(376 \mathrm{MHz}, \mathrm{CDCl}_{3}\right) \delta-62.86 . \mathrm{HRMS}$ (ESI) Calcd for $\left[\mathrm{C}_{18} \mathrm{H}_{17} \mathrm{~F}_{3} \mathrm{NO}_{2} \mathrm{~S}, \mathrm{M}+\mathrm{H}\right]^{+}:$368.0927; found: 368.0932.

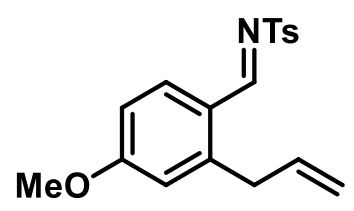

1i: petroleum ether/ethyl acetate $=3: 1(\mathrm{v} / \mathrm{v})$; Pale yellow solid, $1.4 \mathrm{~g}, 76 \%$ yield; m.p. $80-82{ }^{\circ} \mathrm{C}$; ${ }^{1} \mathrm{H}$ NMR $\left(400 \mathrm{MHz}, \mathrm{CDCl}_{3}\right) \delta 9.19(\mathrm{~s}, 1 \mathrm{H}), 8.06(\mathrm{~d}, J=8.8 \mathrm{~Hz}, 1 \mathrm{H}), 7.89-7.82(\mathrm{~m}, 2 \mathrm{H}), 7.33$ $(\mathrm{d}, J=8.0 \mathrm{~Hz}, 2 \mathrm{H}), 6.85-6.75(\mathrm{~m}, 2 \mathrm{H}), 6.05-5.86(\mathrm{~m}, 1 \mathrm{H}), 5.19-5.04(\mathrm{~m}, 1 \mathrm{H}), 5.02-4.89$ 
(m, 1H), $3.87(\mathrm{~s}, 3 \mathrm{H}), 3.68(\mathrm{dt}, J=6.2,1.7 \mathrm{~Hz}, 2 \mathrm{H}), 2.43(\mathrm{~s}, 3 \mathrm{H}) .{ }^{13} \mathrm{C} \mathrm{NMR}\left(101 \mathrm{MHz}, \mathrm{CDCl}_{3}\right)$ $\delta 167.7,165.1,146.7,144.2,136.5,135.9,133.4,129.8,128.0,123.3,117.2,116.1,113.0,55.7$, 37.3, 21.7. HRMS (ESI) $m / z$ calcd for $\left[\mathrm{C}_{18} \mathrm{H}_{19} \mathrm{NO}_{3} \mathrm{SNa}, \mathrm{M}+\mathrm{Na}\right]^{+}: 352.0978$; found: 352.0982 .

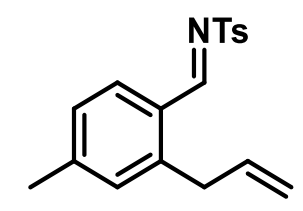

1k: petroleum ether/ethyl acetate $=5: 1(\mathrm{v} / \mathrm{v})$; Yellow solid, $990 \mathrm{mg}, 75 \%$ yield; m.p. $69-71{ }^{\circ} \mathrm{C}$; ${ }^{1} \mathrm{H}$ NMR (400 MHz, $\left.\mathrm{CDCl}_{3}\right) \delta 9.25(\mathrm{~s}, 1 \mathrm{H}), 7.97(\mathrm{~d}, J=8.0 \mathrm{~Hz}, 1 \mathrm{H}), 7.90-7.81(\mathrm{~m}, 2 \mathrm{H}), 7.33$ $(\mathrm{d}, J=8.1 \mathrm{~Hz}, 2 \mathrm{H}), 7.13(\mathrm{~d}, J=8.0 \mathrm{~Hz}, 1 \mathrm{H}), 7.09(\mathrm{~s}, 1 \mathrm{H}), 6.01-5.88(\mathrm{~m}, 1 \mathrm{H}), 5.10-5.05$ (m, $1 \mathrm{H}), 4.97-4.89(\mathrm{~m}, 1 \mathrm{H}), 3.66(\mathrm{dt}, J=6.4,1.7 \mathrm{~Hz}, 2 \mathrm{H}), 2.43(\mathrm{~s}, 3 \mathrm{H}), 2.39$ (s, 3H). ${ }^{13} \mathrm{C} \mathrm{NMR}$ $\left(101 \mathrm{MHz}, \mathrm{CDCl}_{3}\right) \delta 168.4,146.3,144.4,143.9,136.9,135.6,131.8,130.7,129.8,128.2,128.1$, 127.9, 116.9, 37.1, 22.0, 21.8. HRMS (ESI) Calcd for $\left[\mathrm{C}_{18} \mathrm{H}_{20} \mathrm{NO}_{2} \mathrm{~S}, \mathrm{M}+\mathrm{H}\right]^{+}$: 314.1209; found: 314.1212 .

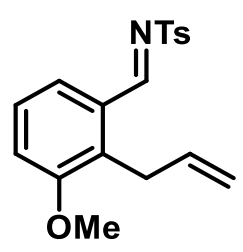

11: petroleum ether/ethyl acetate $=3: 1(\mathrm{v} / \mathrm{v})$; Yellow solid, $533 \mathrm{mg}, 46 \%$ yield; m.p. $116-118{ }^{\circ} \mathrm{C}$; ${ }^{1} \mathrm{H}$ NMR (400 MHz, $\left.\mathrm{CDCl}_{3}\right) \delta 9.29(\mathrm{~s}, 1 \mathrm{H}), 7.90$ - $7.82(\mathrm{~m}, 2 \mathrm{H}), 7.70(\mathrm{dd}, J=7.9,1.1 \mathrm{~Hz}, 1 \mathrm{H})$, $7.34(\mathrm{~d}, J=8.0 \mathrm{~Hz}, 2 \mathrm{H}), 7.30-7.27(\mathrm{~m}, 1 \mathrm{H}), 7.11-7.07(\mathrm{~m}, 1 \mathrm{H}), 6.00-5.86(\mathrm{~m}, 1 \mathrm{H}), 5.00$ $(\mathrm{dq}, J=10.1,1.6 \mathrm{~Hz}, 1 \mathrm{H}), 4.83(\mathrm{dq}, J=17.1,1.7 \mathrm{~Hz}, 1 \mathrm{H}), 3.85(\mathrm{~s}, 3 \mathrm{H}), 3.72(\mathrm{dt}, J=5.9,1.8$ $\mathrm{Hz}, 2 \mathrm{H}), 2.44$ (s, 3H). ${ }^{13} \mathrm{C} \mathrm{NMR}\left(101 \mathrm{MHz}, \mathrm{CDCl}_{3}\right) \delta 168.8,157.6,144.5,136.5,135.1,132.5$, 131.6, 129.8, 128.2, 127.7, 121.5, 116.3, 115.9, 56.0, 28.8, 21.7. HRMS (ESI) Calcd for $\left[\mathrm{C}_{18} \mathrm{H}_{19} \mathrm{NO}_{3} \mathrm{SNa}, \mathrm{M}+\mathrm{Na}\right]^{+}$: 352.0978; found: 352.0981.<smiles></smiles>

1m: petroleum ether/ethyl acetate $=5: 1(\mathrm{v} / \mathrm{v}) ;$ Yellow solid, $704 \mathrm{mg}, 58 \%$ yield; m.p. $87-89{ }^{\circ} \mathrm{C}$; ${ }^{1} \mathrm{H}$ NMR (400 MHz, $\left.\mathrm{CDCl}_{3}\right) \delta 9.23(\mathrm{~s}, 1 \mathrm{H}), 7.91-7.83(\mathrm{~m}, 3 \mathrm{H}), 7.39-7.27(\mathrm{~m}, 4 \mathrm{H}), 5.99$ $5.87(\mathrm{~m}, 1 \mathrm{H}), 5.09-5.03(\mathrm{~m}, 1 \mathrm{H}), 4.92-4.85(\mathrm{~m}, 1 \mathrm{H}), 3.72(\mathrm{dq}, J=5.6,1.7 \mathrm{~Hz}, 2 \mathrm{H}), 2.45(\mathrm{~s}$, $3 \mathrm{H}) .{ }^{13} \mathrm{C}$ NMR $\left(101 \mathrm{MHz}, \mathrm{CDCl}_{3}\right) \delta 167.49,162.24,159.79,144.82,135.39,134.82,132.41$, $130.38,130.21,129.89,128.28,128.21,125.98,121.37,121.14,116.81,28.34,28.29,21.71$. 
${ }^{19} \mathrm{~F} \mathrm{NMR}\left(376 \mathrm{MHz}, \mathrm{CDCl}_{3}\right) \delta-116.5$. HRMS (ESI) $m / z$ calcd for $\left[\mathrm{C}_{17} \mathrm{H}_{16} \mathrm{FNO}_{2} \mathrm{SNa}, \mathrm{M}+\mathrm{Na}\right]^{+}$: 340.0778; found: 340.0782 .<smiles></smiles>

1n: petroleum ether/ethyl acetate $=5: 1(\mathrm{v} / \mathrm{v})$; Yellow solid, $431 \mathrm{mg}, 23 \%$ yield; m.p. $89-92{ }^{\circ} \mathrm{C}$; ${ }^{1} \mathrm{H} \mathrm{NMR}\left(400 \mathrm{MHz}, \mathrm{CDCl}_{3}\right) \delta 9.30(\mathrm{~s}, 1 \mathrm{H}), 7.95(\mathrm{~d}, J=7.8 \mathrm{~Hz}, 1 \mathrm{H}), 7.89-7.82(\mathrm{~m}, 2 \mathrm{H}), 7.40$ (d, $J=7.4 \mathrm{~Hz}, 1 \mathrm{H}), 7.33(\mathrm{~d}, J=8.1 \mathrm{~Hz}, 2 \mathrm{H}), 7.22$ (t, $J=7.7 \mathrm{~Hz}, 1 \mathrm{H}), 6.00-5.89$ (m, 1H), 5.09 - $5.01(\mathrm{~m}, 1 \mathrm{H}), 4.80-4.70(\mathrm{~m}, 1 \mathrm{H}), 3.68(\mathrm{dt}, J=5.4,1.9 \mathrm{~Hz}, 2 \mathrm{H}), 2.43$ (s, 3H), 2.34 (s, 3H). ${ }^{13} \mathrm{C} \mathrm{NMR}\left(101 \mathrm{MHz}, \mathrm{CDCl}_{3}\right) \delta 169.2,144.5,141.7,138.1,136.8,135.7,135.4,130.9,129.9$, 128.2, 127.9, 127.0, 116.7, 32.3, 21.8, 19.7. HRMS (ESI) calcd for $\left[\mathrm{C}_{18} \mathrm{H}_{20} \mathrm{NO}_{2} \mathrm{~S}, \mathrm{M}+\mathrm{H}\right]^{+}$: 314.1209; found: 314.1211 .

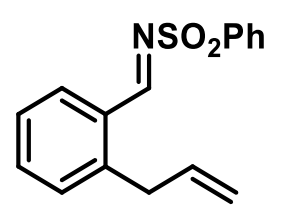

1s: petroleum ether/ethyl acetate $=5: 1(\mathrm{v} / \mathrm{v})$; Pale yellow solid, $1.2 \mathrm{~g}, 64 \%$ yield; m.p. $68-70{ }^{\circ} \mathrm{C}$; ${ }^{1} \mathrm{H}$ NMR $\left(400 \mathrm{MHz}, \mathrm{CDCl}_{3}\right) \delta 9.33(\mathrm{~s}, 1 \mathrm{H}), 8.08(\mathrm{dd}, J=7.9,1.4 \mathrm{~Hz}, 1 \mathrm{H}), 8.02-7.97(\mathrm{~m}, 2 \mathrm{H})$, $7.67-7.50(\mathrm{~m}, 4 \mathrm{H}), 7.37-7.27$ (m, 2H), $6.03-5.89(\mathrm{~m}, 1 \mathrm{H}), 5.14-5.03(\mathrm{~m}, 1 \mathrm{H}), 4.98-4.87$ $(\mathrm{m}, 1 \mathrm{H}), 3.71(\mathrm{td}, J=6.2,1.7 \mathrm{~Hz}, 2 \mathrm{H}) .{ }^{13} \mathrm{C} \mathrm{NMR}\left(101 \mathrm{MHz}, \mathrm{CDCl}_{3}\right) \delta 169.0,143.9,138.3$, 136.8, 135.0, 133.6, 131.1, 130.5, 130.4, 129.2, 128.1, 127.3, 117.1, 37.1. HRMS (ESI) $m / z$ calcd for $\left[\mathrm{C}_{16} \mathrm{H}_{15} \mathrm{NO}_{2} \mathrm{SNa}, \mathrm{M}+\mathrm{Na}\right]^{+}: 308.0716$; found: 308.0720 .

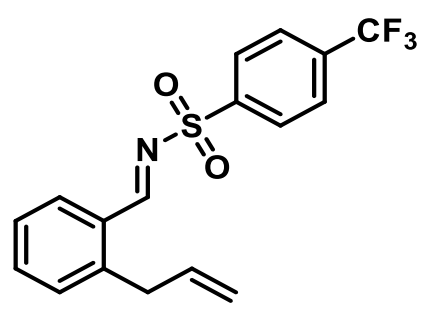

1r: petroleum ether/ethyl acetate $=5: 1(\mathrm{v} / \mathrm{v})$; White solid, $865 \mathrm{mg}$, 36\% yield; m.p. $77-79{ }^{\circ} \mathrm{C}$; ${ }^{1} \mathrm{H}$ NMR $\left(400 \mathrm{MHz}, \mathrm{CDCl}_{3}\right) \delta 9.38(\mathrm{~s}, 1 \mathrm{H}), 8.13(\mathrm{~d}, J=8.2 \mathrm{~Hz}, 2 \mathrm{H}), 8.08(\mathrm{dd}, J=7.9,1.4 \mathrm{~Hz}$, $1 \mathrm{H}), 7.82(\mathrm{~d}, J=8.3 \mathrm{~Hz}, 2 \mathrm{H}), 7.56(\mathrm{td}, J=7.6,1.5 \mathrm{~Hz}, 1 \mathrm{H}), 7.38-7.28(\mathrm{~m}, 2 \mathrm{H}), 6.06-5.91$ $(\mathrm{m}, 1 \mathrm{H}), 5.15-5.06(\mathrm{~m}, 1 \mathrm{H}), 4.99-4.88(\mathrm{~m}, 1 \mathrm{H}), 3.73(\mathrm{dt}, J=6.3,1.8 \mathrm{~Hz}, 2 \mathrm{H}) .{ }^{13} \mathrm{C} \mathrm{NMR}$ $\left(101 \mathrm{MHz}, \mathrm{CDCl}_{3}\right) \delta 170.3,144.2,142.2,136.9,135.5,135.1,131.3,130.7,130.3,128.7,127.5$, $126.4\left(\mathrm{q}, J_{\mathrm{C}, \mathrm{F}}=3.5 \mathrm{~Hz}\right), 117.2,37.2 .{ }^{19} \mathrm{~F}$ NMR $\left(376 \mathrm{MHz}, \mathrm{CDCl}_{3}\right) \delta-63.20$. HRMS (ESI) $\mathrm{m} / z$ calcd for $\left[\mathrm{C}_{17} \mathrm{H}_{14} \mathrm{~F}_{3} \mathrm{NO}_{2} \mathrm{SNa}, \mathrm{M}+\mathrm{Na}\right]^{+}$: 376.0590; found: 376.0593 . 


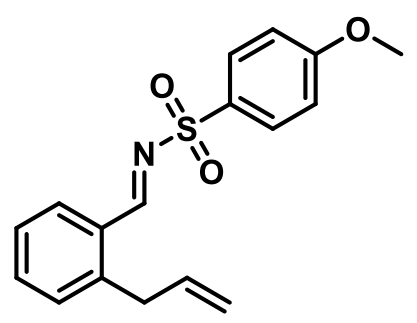

1t: petroleum ether/ethyl acetate $=3: 1(\mathrm{v} / \mathrm{v})$; Yellow solid, $2.2 \mathrm{~g}, 69 \%$ yield; m.p. $57-59{ }^{\circ} \mathrm{C} ;{ }^{1} \mathrm{H}$ NMR (400 MHz, $\left.\mathrm{CDCl}_{3}\right) \delta 9.28(\mathrm{~s}, 1 \mathrm{H}), 8.07(\mathrm{dd}, J=7.9,1.4 \mathrm{~Hz}, 1 \mathrm{H}), 7.92(\mathrm{~d}, J=9.0 \mathrm{~Hz}, 2 \mathrm{H})$, $7.52(\mathrm{td}, J=7.5,1.5 \mathrm{~Hz}, 1 \mathrm{H}), 7.35-7.26(\mathrm{~m}, 2 \mathrm{H}), 7.01(\mathrm{~d}, J=9.0 \mathrm{~Hz}, 2 \mathrm{H}), 5.96$ (ddt, $J=17.1$, $10.1,6.1 \mathrm{~Hz}, 1 \mathrm{H}), 5.09(\mathrm{dq}, J=10.1,1.5 \mathrm{~Hz}, 1 \mathrm{H}), 4.92(\mathrm{dq}, J=17.1,1.7 \mathrm{~Hz}, 1 \mathrm{H}), 3.88(\mathrm{~s}, 3 \mathrm{H})$, $3.70(\mathrm{dt}, J=6.2,1.7 \mathrm{~Hz}, 2 \mathrm{H}) .{ }^{13} \mathrm{C} \mathrm{NMR}\left(101 \mathrm{MHz}, \mathrm{CDCl}_{3}\right) \delta 168.0,163.8,143.6,136.8,134.8$, 131.1, 130.5, 130.4, 130.3, 129.7, 127.3, 117.0, 114.5, 55.8, 37.1. HRMS (ESI) $\mathrm{m} / \mathrm{z}$ calcd for $\left[\mathrm{C}_{17} \mathrm{H}_{17} \mathrm{NO}_{3} \mathrm{SNa}, \mathrm{M}+\mathrm{Na}\right]^{+}$: 338.0821; found: 338.0826 .

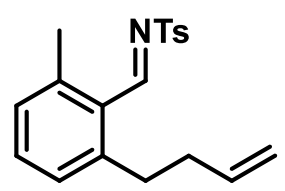

4d: petroleum ether/ethyl acetate $=5: 1(\mathrm{v} / \mathrm{v})$; Yellow oil, $720 \mathrm{mg}, 48 \%$ yield; ${ }^{1} \mathrm{H}$ NMR (400 $\left.\mathrm{MHz}, \mathrm{CDCl}_{3}\right) \delta 9.45(\mathrm{~s}, 1 \mathrm{H}), 7.91-7.85(\mathrm{~m}, 2 \mathrm{H}), 7.37-7.30(\mathrm{~m}, 3 \mathrm{H}), 7.14-7.07(\mathrm{~m}, 2 \mathrm{H})$, $5.73(\mathrm{ddt}, J=16.9,10.3,6.6 \mathrm{~Hz}, 1 \mathrm{H}), 5.00-4.91(\mathrm{~m}, 2 \mathrm{H}), 3.01-2.90(\mathrm{~m}, 2 \mathrm{H}), 2.56(\mathrm{~s}, 3 \mathrm{H})$, $2.44(\mathrm{~s}, 3 \mathrm{H}), 2.28-2.17(\mathrm{~m}, 2 \mathrm{H}) .{ }^{13} \mathrm{C} \mathrm{NMR}\left(101 \mathrm{MHz}, \mathrm{CDCl}_{3}\right) \delta 169.2,146.1,144.6,142.5$, 137.4, 135.5, 133.4, 130.0, 129.9, 129.2, 128.6, 128.1, 115.4, 36.0, 33.9, 21.9, 21.7. HRMS (ESI) $m / z$ calcd for $\left[\mathrm{C}_{19} \mathrm{H}_{22} \mathrm{NO}_{2} \mathrm{~S}, \mathrm{M}+\mathrm{H}\right]^{+}: 328.1366$; found: 328.1368 .

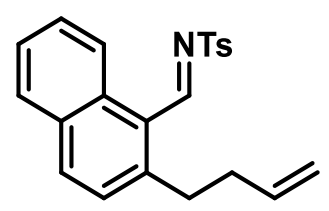

4e: petroleum ether/ethyl acetate $=5: 1(\mathrm{v} / \mathrm{v})$; Yellow solid, $1.1 \mathrm{~g}, 73 \%$ yield; m.p. $94-96{ }^{\circ} \mathrm{C}$; ${ }^{1} \mathrm{H}$ NMR $\left(400 \mathrm{MHz}, \mathrm{CDCl}_{3}\right) \delta 9.81(\mathrm{~s}, 1 \mathrm{H}), 8.92(\mathrm{~d}, J=8.6 \mathrm{~Hz}, 1 \mathrm{H}), 8.00-7.91(\mathrm{~m}, 3 \mathrm{H}), 7.86$ - $7.81(\mathrm{~m}, 1 \mathrm{H}), 7.64-7.57(\mathrm{~m}, 1 \mathrm{H}), 7.56-7.48(\mathrm{~m}, 1 \mathrm{H}), 7.39-7.34(\mathrm{~m}, 3 \mathrm{H}), 5.78$ (ddt, $J=$ 17.0, 10.2, 6.7 Hz, 1H), $5.04-4.91(\mathrm{~m}, 2 \mathrm{H}), 3.16-3.08(\mathrm{~m}, 2 \mathrm{H}), 2.44(\mathrm{~s}, 3 \mathrm{H}), 2.40-2.30$ (m, 2H). ${ }^{13} \mathrm{C}$ NMR $\left(101 \mathrm{MHz}, \mathrm{CDCl}_{3}\right) \delta 168.9,147.7,144.7,136.8,135.4,135.0,132.6,132.2$, 130.0, 128.9, 128.8, 128.8, 128.2, 126.4, 125.1, 124.9, 116.1, 36.5, 34.1, 21.8. HRMS (ESI) $m / z$ calcd for $\left[\mathrm{C}_{22} \mathrm{H}_{21} \mathrm{NO}_{2} \mathrm{SNa}, \mathrm{M}+\mathrm{Na}\right]^{+}: 386.1185$; found: 386.1189 . 


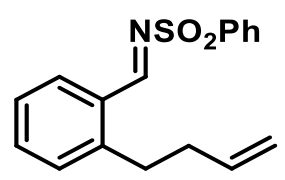

4h: petroleum ether/ethyl acetate $=5: 1(\mathrm{v} / \mathrm{v})$; Orange oil, $1.3 \mathrm{~g}$, 90\% yield; ${ }^{1} \mathrm{H}$ NMR $(400 \mathrm{MHz}$, $\left.\mathrm{CDCl}_{3}\right) \delta 9.32(\mathrm{~s}, 1 \mathrm{H}), 8.07-7.99(\mathrm{~m}, 3 \mathrm{H}), 7.67-7.60(\mathrm{~m}, 1 \mathrm{H}), 7.59-7.49(\mathrm{~m}, 3 \mathrm{H}), 7.34-$ $7.27(\mathrm{~m}, 2 \mathrm{H}), 5.78(\mathrm{ddt}, J=17.1,10.4,6.7 \mathrm{~Hz}, 1 \mathrm{H}), 5.03-4.95(\mathrm{~m}, 2 \mathrm{H}), 3.03(\mathrm{dd}, J=8.6,6.8$ $\mathrm{Hz}, 2 \mathrm{H}), 2.36-2.26(\mathrm{~m}, 2 \mathrm{H}) .{ }^{13} \mathrm{C} \mathrm{NMR}\left(101 \mathrm{MHz}, \mathrm{CDCl}_{3}\right) \delta 169.0,146.2,138.4,136.8,134.8$, 133.6, 131.1, 131.0, 130.0, 129.3, 128.1, 127.0, 116.2, 36.2, 32.5. HRMS (ESI) $\mathrm{m} / z$ calcd for $\left[\mathrm{C}_{17} \mathrm{H}_{18} \mathrm{NO}_{2} \mathrm{~S}, \mathrm{M}+\mathrm{H}\right]^{+}:$300.1053; found: 300.1055.

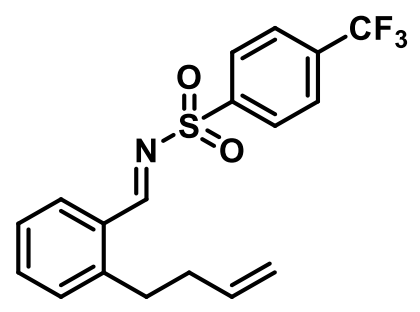

4j: petroleum ether/ethyl acetate $=5: 1(\mathrm{v} / \mathrm{v}) ;$ Pale yellow oil, $1.3 \mathrm{~g}, 73 \%$ yield; ${ }^{1} \mathrm{H}$ NMR $(400$ $\left.\mathrm{MHz}, \mathrm{CDCl}_{3}\right) \delta 9.37(\mathrm{~s}, 1 \mathrm{H}), 8.15(\mathrm{~d}, J=8.2 \mathrm{~Hz}, 2 \mathrm{H}), 8.04(\mathrm{dd}, J=7.8,1.4 \mathrm{~Hz}, 1 \mathrm{H}), 7.82(\mathrm{~d}, J$ $=8.3 \mathrm{~Hz}, 2 \mathrm{H}), 7.55(\mathrm{td}, J=7.6,1.5 \mathrm{~Hz}, 1 \mathrm{H}), 7.36-7.28(\mathrm{~m}, 2 \mathrm{H}), 5.78$ (ddt, $J=17.1,10.4,6.7$ $\mathrm{Hz}, 1 \mathrm{H}), 5.05-4.95(\mathrm{~m}, 2 \mathrm{H}), 3.11-2.99(\mathrm{~m}, 2 \mathrm{H}), 2.39-2.27(\mathrm{~m}, 2 \mathrm{H}) .{ }^{13} \mathrm{C} \mathrm{NMR}(101 \mathrm{MHz}$, $\left.\mathrm{CDCl}_{3}\right) \delta 170.2,146.7,142.3,136.7,135.3,131.3,131.2,129.8,128.6,127.2,127.1,126.4(\mathrm{q}$, $\left.J_{\mathrm{C}, \mathrm{F}}=3.6 \mathrm{~Hz}\right), 116.3,36.2,32.5 .{ }^{19} \mathrm{~F} \mathrm{NMR}\left(376 \mathrm{MHz}, \mathrm{CDCl}_{3}\right) \delta-63.20$. HRMS (ESI) $\mathrm{m} / z$ calcd for $\left[\mathrm{C}_{18} \mathrm{H}_{16} \mathrm{~F}_{3} \mathrm{NO}_{2} \mathrm{SNa}, \mathrm{M}+\mathrm{Na}\right]^{+}$: 390.0746, found: 390.0750 .

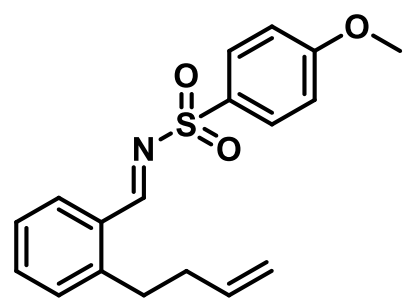

4i: petroleum ether/ethyl acetate $=3: 1(\mathrm{v} / \mathrm{v})$; Orange oil, $1.9 \mathrm{~g}, 97 \%$ yield; ${ }^{1} \mathrm{H}$ NMR $(400 \mathrm{MHz}$, $\left.\mathrm{CDCl}_{3}\right) \delta 9.27(\mathrm{~s}, 1 \mathrm{H}), 8.03(\mathrm{dd}, J=7.9,1.4 \mathrm{~Hz}, 1 \mathrm{H}), 7.97-7.90(\mathrm{~m}, 2 \mathrm{H}), 7.50(\mathrm{td}, J=7.6,1.5$ $\mathrm{Hz}, 1 \mathrm{H}), 7.34-7.25(\mathrm{~m}, 2 \mathrm{H}), 7.05-6.98(\mathrm{~m}, 2 \mathrm{H}), 5.78$ (ddt, $J=17.0,10.3,6.7 \mathrm{~Hz}, 1 \mathrm{H}), 5.05$ $-4.94(\mathrm{~m}, 2 \mathrm{H}), 3.88(\mathrm{~s}, 3 \mathrm{H}), 3.07-2.98(\mathrm{~m}, 2 \mathrm{H}), 2.36-2.25(\mathrm{~m}, 2 \mathrm{H}) .{ }^{13} \mathrm{C} \mathrm{NMR}(101 \mathrm{MHz}$, $\left.\mathrm{CDCl}_{3}\right) \delta 167.9,163.7,145.9,136.8,134.5,130.9,130.7,130.2,130.0,129.6,126.9,116.0$, 114.5, 55.7, 36.1, 32.4. HRMS (ESI) $m / z$ calcd for $\left[\mathrm{C}_{18} \mathrm{H}_{19} \mathrm{NO}_{3} \mathrm{SNa}, \mathrm{M}+\mathrm{Na}\right]^{+}:$352.0978; found: 352.0983 . 


\section{Nickel-Catalyzed Intramolecular Hydroalkenylation of Imines}

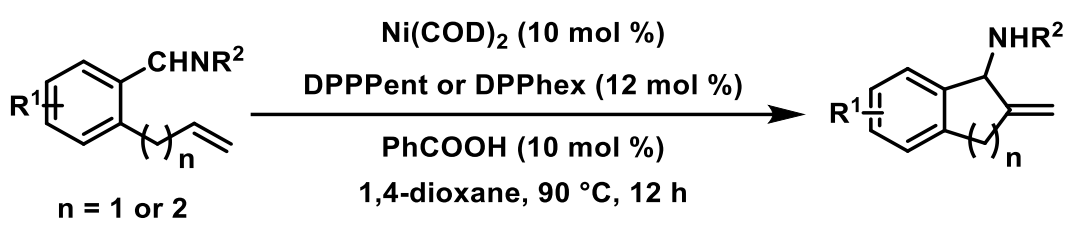

To an oven-dried sealed tube, imine ( $0.2 \mathrm{mmol}, 1$ equiv $)$ and $\mathrm{PhCOOH} \mathrm{(0.02} \mathrm{mmol,} 0.1$ equiv) were added. The DPPPent (or DPPhex, $0.024 \mathrm{mmol}, 0.12$ equiv), $\mathrm{Ni}(\mathrm{COD})_{2}(0.02 \mathrm{mmol}, 0.1$ equiv) and 1,4-dioxane (1 mL) were added. Sealed the tube and moved it out from the glovebox. The mixture was heated at $90{ }^{\circ} \mathrm{C}$ using oil bath for $12 \mathrm{~h}$. After cooling to room temperature, one drop of water was added to quench the reaction. The solvent was removed under reduced pressure and the residue was purified by flash chromatography to afford the pure product. This procedure can be scaled up to gram scale at the same concentration, see $\mathbf{2 q}$.

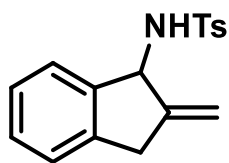

2a: petroleum ether/ethyl acetate $=5: 1(\mathrm{v} / \mathrm{v})$; Yellow solid, $50.3 \mathrm{mg}, 83 \%$ yield; m.p. 128 $130{ }^{\circ} \mathrm{C} .{ }^{1} \mathrm{H}$ NMR $\left(400 \mathrm{MHz}, \mathrm{CDCl}_{3}\right) \delta 7.88(\mathrm{~d}, J=8.3 \mathrm{~Hz}, 2 \mathrm{H}), 7.35(\mathrm{~d}, J=8.0 \mathrm{~Hz}, 2 \mathrm{H}), 7.25$ $-7.13(\mathrm{~m}, 3 \mathrm{H}), 7.09$ (d, $J=7.5 \mathrm{~Hz}, 1 \mathrm{H}), 5.32(\mathrm{~d}, J=9.4 \mathrm{~Hz}, 1 \mathrm{H}), 5.14$ (q, $J=2.3 \mathrm{~Hz}, 1 \mathrm{H}), 5.09$ $(\mathrm{q}, J=2.4 \mathrm{~Hz}, 1 \mathrm{H}), 4.77(\mathrm{~d}, J=9.3 \mathrm{~Hz}, 1 \mathrm{H}), 3.58(\mathrm{~s}, 2 \mathrm{H}), 2.46(\mathrm{~s}, 3 \mathrm{H}) .{ }^{13} \mathrm{C} \mathrm{NMR}(101 \mathrm{MHz}$, $\left.\mathrm{CDCl}_{3}\right) \delta 149.9,143.7,141.8,140.5,138.6,130.0,128.7,127.4,127.3,124.9,124.7,111.0$, 60.4, 36.9, 21.7. HRMS (ESI) $\mathrm{m} / z$ calcd for $\left[\mathrm{C}_{17} \mathrm{H}_{17} \mathrm{NO}_{2} \mathrm{SNa}, \mathrm{M}+\mathrm{Na}\right]^{+}$: 322.0872 , found: 322.0878 .

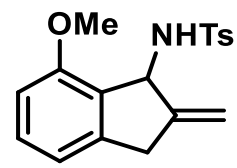

2b: petroleum ether/ethyl acetate $=5: 1(\mathrm{v} / \mathrm{v}) ;$ Yellow solid, $54.3 \mathrm{mg}, 82 \%$ yield; m.p. $121-$ $123{ }^{\circ} \mathrm{C} ;{ }^{1} \mathrm{H}$ NMR $\left(400 \mathrm{MHz}, \mathrm{CDCl}_{3}\right) \delta 7.76(\mathrm{~d}, J=8.2 \mathrm{~Hz}, 2 \mathrm{H}), 7.27(\mathrm{~d}, J=8.0 \mathrm{~Hz}, 2 \mathrm{H}), 7.18$ (t, $J=7.9 \mathrm{~Hz}, 1 \mathrm{H}), 6.78(\mathrm{~d}, J=7.5 \mathrm{~Hz}, 1 \mathrm{H}), 6.55(\mathrm{~d}, J=8.2 \mathrm{~Hz}, 1 \mathrm{H}), 5.54(\mathrm{q}, J=2.2 \mathrm{~Hz}, 1 \mathrm{H})$, 5.32-5.21 (m, 2H), $4.81(\mathrm{~d}, J=6.5 \mathrm{~Hz}, 1 \mathrm{H}), 3.81-3.38(\mathrm{~m}, 5 \mathrm{H}), 2.43(\mathrm{~s}, 3 \mathrm{H}) .{ }^{13} \mathrm{C}$ NMR $(101$ $\left.\mathrm{MHz}, \mathrm{CDCl}_{3}\right) \delta 156.5,148.6,144.0,142.8,138.9,130.6,129.2,127.5,127.5,116.9,113.1$, 108.4, 59.1, 54.7, 37.3, 21.6. HRMS (ESI) $\mathrm{m} / z$ calcd for $\left[\mathrm{C}_{18} \mathrm{H}_{19} \mathrm{NO}_{3} \mathrm{SNa}, \mathrm{M}+\mathrm{H}\right]^{+}: 352.0978$, found: 352.0983 . 


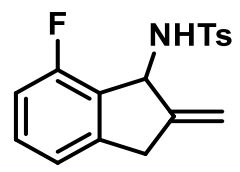

2c: petroleum ether/ethyl acetate $=5: 1(\mathrm{v} / \mathrm{v})$; Yellow solid, $47.2 \mathrm{mg}, 74 \%$ yield; m.p. $104-$ $106{ }^{\circ} \mathrm{C} ;{ }^{1} \mathrm{H}$ NMR $\left(400 \mathrm{MHz}, \mathrm{CDCl}_{3}\right) \delta 7.81(\mathrm{~d}, J=8.0 \mathrm{~Hz}, 2 \mathrm{H}), 7.30(\mathrm{~d}, J=8.0 \mathrm{~Hz}, 2 \mathrm{H}), 7.25$ $-7.18(\mathrm{~m}, 1 \mathrm{H}), 6.98(\mathrm{~d}, J=7.5 \mathrm{~Hz}, 1 \mathrm{H}), 6.78(\mathrm{t}, J=8.8 \mathrm{~Hz}, 1 \mathrm{H}), 5.51-5.42(\mathrm{~m}, 2 \mathrm{H}), 5.27$ (q, $J=2.2 \mathrm{~Hz}, 1 \mathrm{H}), 4.74(\mathrm{~d}, J=8.4 \mathrm{~Hz}, 1 \mathrm{H}), 3.79-3.50(\mathrm{~m}, 2 \mathrm{H}), 2.44(\mathrm{~s}, 3 \mathrm{H}) .{ }^{13} \mathrm{C}$ NMR $(101$ $\left.\mathrm{MHz}, \mathrm{CDCl}_{3}\right) \delta 161.0,158.5,148.6,143.4,138.4,130.9,130.8,129.6,129.5,127.5,127.4$, 120.6, 120.5, 114.1, 113.9, 113.1, 58.3, 37.1, 21.7. $\left.{ }^{19} \mathrm{~F} \mathrm{NMR} \mathrm{(376} \mathrm{MHz,} \mathrm{CDCl}_{3}\right) \delta-117.56$. HRMS (ESI) $m / z$ calcd for $\left[\mathrm{C}_{17} \mathrm{H}_{15} \mathrm{FNO}_{2} \mathrm{~S}, \mathrm{M}-\mathrm{H}\right]^{-}: 316.0813$, found: 316.0810 .

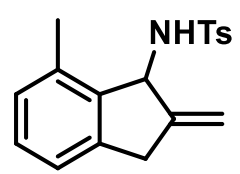

2d: petroleum ether/ethyl acetate $=5: 1(\mathrm{v} / \mathrm{v})$; Yellow solid, $43.5 \mathrm{mg}$, $69 \%$ yield; m.p.119$121{ }^{\circ} \mathrm{C} ;{ }^{1} \mathrm{H} \mathrm{NMR}\left(400 \mathrm{MHz}, \mathrm{CDCl}_{3}\right) \delta 7.76(\mathrm{~d}, J=8.1 \mathrm{~Hz}, 2 \mathrm{H}), 7.28(\mathrm{~d}, J=7.9 \mathrm{~Hz}, 2 \mathrm{H}), 7.14$ $(\mathrm{t}, J=7.5 \mathrm{~Hz}, 1 \mathrm{H}), 7.01(\mathrm{~d}, J=7.6 \mathrm{~Hz}, 1 \mathrm{H}), 6.96(\mathrm{~d}, J=7.5 \mathrm{~Hz}, 1 \mathrm{H}), 5.36(\mathrm{~d}, J=8.6 \mathrm{~Hz}, 1 \mathrm{H})$, $5.03(\mathrm{q}, J=2.0 \mathrm{~Hz}, 1 \mathrm{H}), 4.94(\mathrm{q}, J=2.1 \mathrm{~Hz}, 1 \mathrm{H}), 4.62(\mathrm{~d}, J=8.5 \mathrm{~Hz}, 1 \mathrm{H}), 3.71-3.36(\mathrm{~m}, 2 \mathrm{H})$, $2.43(\mathrm{~s}, 3 \mathrm{H}), 2.21$ (s, 3H). ${ }^{13} \mathrm{C} \mathrm{NMR}\left(101 \mathrm{MHz}, \mathrm{CDCl}_{3}\right) \delta 148.6,143.4,141.9,139.2,138.9$, 136.1, 129.7, 129.2, 128.9, 127.2, 122.2, 112.5, 60.4, 37.1, 21.7, 18.6. HRMS (ESI) $\mathrm{m} / \mathrm{z}$ calcd for $\left[\mathrm{C}_{18} \mathrm{H}_{18} \mathrm{NO}_{2} \mathrm{~S}, \mathrm{M}-\mathrm{H}\right]^{-}: 312.1064$, found: 312.1061 .

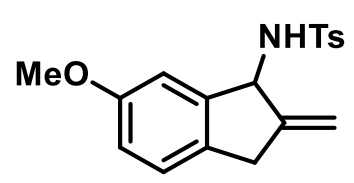

2e: petroleum ether/ethyl acetate $=3: 1(\mathrm{v} / \mathrm{v})$; Yellow solid, $53.7 \mathrm{mg}, 81 \%$ yield; m.p. 152$154{ }^{\circ} \mathrm{C} ;{ }^{1} \mathrm{H}$ NMR $\left(400 \mathrm{MHz}, \mathrm{CDCl}_{3}\right) \delta 7.89(\mathrm{~d}, J=8.3 \mathrm{~Hz}, 2 \mathrm{H}), 7.36(\mathrm{~d}, J=8.0 \mathrm{~Hz}, 2 \mathrm{H}), 7.06$ $(\mathrm{d}, J=8.3 \mathrm{~Hz}, 1 \mathrm{H}), 6.77(\mathrm{dd}, J=8.3,2.5 \mathrm{~Hz}, 1 \mathrm{H}), 6.50(\mathrm{~d}, J=2.5 \mathrm{~Hz}, 1 \mathrm{H}), 5.29$ (d, $J=9.4 \mathrm{~Hz}$, $1 \mathrm{H}), 5.13(\mathrm{q}, J=2.3 \mathrm{~Hz}, 1 \mathrm{H}), 5.09$ (q, $J=2.3 \mathrm{~Hz}, 1 \mathrm{H}), 4.80$ (d, $J=9.3 \mathrm{~Hz}, 1 \mathrm{H}), 3.65$ (s, 3H), 3.50 (s, 2H), 2.45 (s, 3H). ${ }^{13} \mathrm{C}$ NMR (101 MHz, $\left.\mathrm{CDCl}_{3}\right) \delta 159.5,150.4,143.7,143.0,138.8$, 132.3, 130.0, 127.3, 125.5, 116.2, 110.9, 108.7, 60.7, 55.5, 36.1, 21.7. HRMS (ESI) $\mathrm{m} / \mathrm{z}$ calcd for $\left[\mathrm{C}_{18} \mathrm{H}_{19} \mathrm{NO}_{3} \mathrm{SNa}, \mathrm{M}+\mathrm{Na}\right]^{+}: 352.0978$; found: 352.0982 .

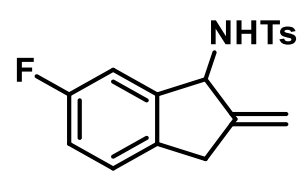

2f: petroleum ether/ethyl acetate $=5: 1(\mathrm{v} / \mathrm{v})$; Yellow solid, $43.2 \mathrm{mg}, 68 \%$ yield; m.p. $138-$ 
$140{ }^{\circ} \mathrm{C} ;{ }^{1} \mathrm{H} \mathrm{NMR}\left(400 \mathrm{MHz}, \mathrm{CDCl}_{3}\right) \delta 7.87(\mathrm{~d}, J=8.0 \mathrm{~Hz}, 2 \mathrm{H}), 7.36(\mathrm{~d}, J=8.0 \mathrm{~Hz}, 2 \mathrm{H}), 7.11$ $(\mathrm{dd}, J=8.4,5.1 \mathrm{~Hz}, 1 \mathrm{H}), 6.91(\mathrm{td}, J=8.7,2.5 \mathrm{~Hz}, 1 \mathrm{H}), 6.76(\mathrm{dd}, J=8.5,2.5 \mathrm{~Hz}, 1 \mathrm{H}), 5.29$ (d, $J=9.4 \mathrm{~Hz}, 1 \mathrm{H}), 5.14(\mathrm{q}, J=2.4 \mathrm{~Hz}, 1 \mathrm{H}), 5.08(\mathrm{q}, J=2.4 \mathrm{~Hz}, 1 \mathrm{H}), 4.78(\mathrm{~d}, J=9.3 \mathrm{~Hz}, 1 \mathrm{H})$, 3.53 (s, 2H), 2.47 (s, 3H). ${ }^{13} \mathrm{C}$ NMR (101 MHz, $\left.\mathrm{CDCl}_{3}\right) \delta 163.7,161.3,149.8,143.9,143.9$, 143.8, 138.4, 135.7, 130.1, 127.2, 125.9, 125.8, 116.0, 115.8, 112.0, 111.8, 111.3, 60.4, 36.2, 21.7. $\left.{ }^{19} \mathrm{~F} \mathrm{NMR} \mathrm{(376} \mathrm{MHz,} \mathrm{CDCl}_{3}\right) \delta-114.96$. HRMS (ESI) $\mathrm{m} / z$ calcd for $\left[\mathrm{C}_{17} \mathrm{H}_{15} \mathrm{FNO}_{2} \mathrm{~S}, \mathrm{M}-\right.$ $\mathrm{H}]^{-}:$316.0813; found: 316.0895 .

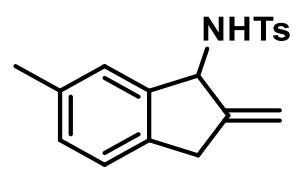

2g: petroleum ether/ethyl acetate $=5: 1(\mathrm{v} / \mathrm{v})$; Yellow solid, $49.2 \mathrm{mg}, 78 \%$ yield; m.p. $121-$ $123{ }^{\circ} \mathrm{C} ;{ }^{1} \mathrm{H} \mathrm{NMR}\left(400 \mathrm{MHz}, \mathrm{CDCl}_{3}\right) \delta 7.87(\mathrm{~d}, J=7.9 \mathrm{~Hz}, 2 \mathrm{H}), 7.35(\mathrm{~d}, J=7.9 \mathrm{~Hz}, 2 \mathrm{H}), 7.08-$ $6.96(\mathrm{~m}, 2 \mathrm{H}), 6.77(\mathrm{~s}, 1 \mathrm{H}), 5.26(\mathrm{~d}, J=9.3 \mathrm{~Hz}, 1 \mathrm{H}), 5.13-5.07(\mathrm{~m}, 1 \mathrm{H}), 5.06-5.00(\mathrm{~m}, 1 \mathrm{H})$, $4.79(\mathrm{~d}, J=9.3 \mathrm{~Hz}, 1 \mathrm{H}), 3.51(\mathrm{~s}, 2 \mathrm{H}), 2.46(\mathrm{~s}, 3 \mathrm{H}), 2.23(\mathrm{~s}, 3 \mathrm{H}),{ }^{13} \mathrm{C} \mathrm{NMR}\left(101 \mathrm{MHz}, \mathrm{CDCl}_{3}\right)$ $\delta 150.2,143.6,141.8,138.8,137.4,137.1,129.9,129.5,127.3,125.4,124.4,110.8,60.4,36.5$, 21.7, 21.3. HRMS (ESI) Calcd for $\left[\mathrm{C}_{18} \mathrm{H}_{19} \mathrm{NO}_{2} \mathrm{SNa}, \mathrm{M}+\mathrm{Na}\right]^{+}:$336.1029; found: 336.1032.

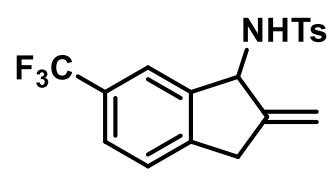

2h: petroleum ether/ethyl acetate $=5: 1(\mathrm{v} / \mathrm{v})$; Yellow solid, $36.4 \mathrm{mg}$, 50\% yield; m.p. $152-$ $154{ }^{\circ} \mathrm{C} ;{ }^{1} \mathrm{H}$ NMR $\left(400 \mathrm{MHz}, \mathrm{CDCl}_{3}\right) \delta 7.87(\mathrm{~d}, J=7.9 \mathrm{~Hz}, 2 \mathrm{H}), 7.45(\mathrm{~d}, J=8.0 \mathrm{~Hz}, 1 \mathrm{H}), 7.36$ $(\mathrm{d}, J=8.0 \mathrm{~Hz}, 2 \mathrm{H}), 7.27(\mathrm{~d}, J=7.5 \mathrm{~Hz}, 1 \mathrm{H}), 7.00(\mathrm{~s}, 1 \mathrm{H}), 5.34(\mathrm{~d}, J=9.4 \mathrm{~Hz}, 1 \mathrm{H}), 5.24-5.15$ $(\mathrm{m}, 2 \mathrm{H}), 4.95(\mathrm{~d}, J=9.3 \mathrm{~Hz}, 1 \mathrm{H}), 3.62(\mathrm{~s}, 2 \mathrm{H}), 2.47$ (s, 3H). ${ }^{13} \mathrm{C} \mathrm{NMR}\left(101 \mathrm{MHz}, \mathrm{CDCl}_{3}\right) \delta$ $148.7,144.5,144.2,142.7,138.4,130.2,127.2,125.6\left(\mathrm{q}, J_{\mathrm{C}, \mathrm{F}}=3.9 \mathrm{~Hz}\right), 125.1,122.0\left(\mathrm{q}, J_{\mathrm{C}, \mathrm{F}}=\right.$ 3.7 Hz), 111.9, 60.1, 36.8, 21.6. $\left.{ }^{19} \mathrm{~F} \mathrm{NMR} \mathrm{(376} \mathrm{MHz,} \mathrm{CDCl}_{3}\right) \delta-62.86$. HRMS (ESI) $\mathrm{m} / z$ calcd for $\left[\mathrm{C}_{18} \mathrm{H}_{16} \mathrm{~F}_{3} \mathrm{NO}_{2} \mathrm{SNa}, \mathrm{M}+\mathrm{Na}\right]^{+}$: 390.0746, found: 390.0750 .

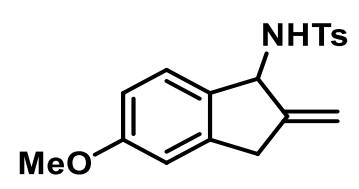

2i: petroleum ether/ethyl acetate $=3: 1(\mathrm{v} / \mathrm{v})$; Yellow solid, $55.6 \mathrm{mg}, 84 \%$ yield; ${ }^{1} \mathrm{H}$ NMR (400 $\left.\mathrm{MHz}, \mathrm{CDCl}_{3}\right) \delta 7.87(\mathrm{~d}, J=8.2 \mathrm{~Hz}, 2 \mathrm{H}), 7.35(\mathrm{~d}, J=7.9 \mathrm{~Hz}, 2 \mathrm{H}), 6.96(\mathrm{~d}, J=8.2 \mathrm{~Hz}, 1 \mathrm{H}), 6.73$ $-6.67(\mathrm{~m}, 2 \mathrm{H}), 5.24(\mathrm{~d}, J=9.3 \mathrm{~Hz}, 1 \mathrm{H}), 5.12(\mathrm{q}, J=2.3 \mathrm{~Hz}, 1 \mathrm{H}), 5.08(\mathrm{q}, J=2.3 \mathrm{~Hz}, 1 \mathrm{H}), 4.75$ $(\mathrm{d}, J=9.2 \mathrm{~Hz}, 1 \mathrm{H}), 3.77(\mathrm{~s}, 3 \mathrm{H}), 3.54(\mathrm{~s}, 2 \mathrm{H}), 2.46(\mathrm{~s}, 3 \mathrm{H}) .{ }^{13} \mathrm{C} \mathrm{NMR}\left(101 \mathrm{MHz}, \mathrm{CDCl}_{3}\right) \delta$ $160.3,150.1$, 143.6, 142.1, 138.7, 133.8, 129.9, 127.2, 125.8, 113.8, 111.0, 109.6, 59.9, 55.5, 
37.0, 21.7. HRMS (ESI) $m / z$ Calcd for $\left[\mathrm{C}_{18} \mathrm{H}_{19} \mathrm{NO}_{3} \mathrm{SNa}, \mathrm{M}+\mathrm{Na}\right]^{+}: 352.0978$; found: 352.0981 .<smiles>C=C1Cc2cc(F)ccc2C1N[AsH3]</smiles>

2j: petroleum ether/ethyl acetate $=5: 1(\mathrm{v} / \mathrm{v})$; yellow solid, $54.1 \mathrm{mg}, 85 \%$ yield; m.p. $87-89{ }^{\circ} \mathrm{C}$; ${ }^{1} \mathrm{H}$ NMR $\left(400 \mathrm{MHz}, \mathrm{CDCl}_{3}\right) \delta 7.84(\mathrm{~d}, J=7.9 \mathrm{~Hz}, 2 \mathrm{H}), 7.33(\mathrm{~d}, J=7.9 \mathrm{~Hz}, 2 \mathrm{H}), 7.12-7.02(\mathrm{~m}$, $1 \mathrm{H}), 6.89-6.79(\mathrm{~m}, 2 \mathrm{H}), 5.31-4.93(\mathrm{~m}, 4 \mathrm{H}), 3.54(\mathrm{~s}, 2 \mathrm{H}), 2.45(\mathrm{~s}, 3 \mathrm{H}) .{ }^{13} \mathrm{C}$ NMR $(101 \mathrm{MHz}$, $\left.\mathrm{CDCl}_{3}\right) \delta 164.4,161.9,149.4,143.7,142.6,142.5,138.4,137.4,137.4,129.9,129.8,127.1$, 126.5, 126.4, 114.7, 114.5, 111.6, 111.4, 111.3, 59.6, 36.8, 21.7. ${ }^{19} \mathrm{~F}$ NMR (376 MHz, $\left.\mathrm{CDCl}_{3}\right)$ $\delta$-113.63. HRMS (ESI) $\mathrm{m} / \mathrm{z}$ calcd for $\left[\mathrm{C}_{17} \mathrm{H}_{15} \mathrm{FNO}_{2} \mathrm{~S}, \mathrm{M}-\mathrm{H}\right]^{-}: 316.0813$; found: 316.0810 .

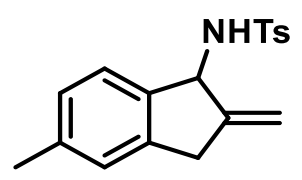

2k: petroleum ether/ethyl acetate $=5: 1(\mathrm{v} / \mathrm{v})$; yellow solid, $44.6 \mathrm{mg}, 71 \%$ yield; ${ }^{1} \mathrm{H}$ NMR $(400$ $\left.\mathrm{MHz}, \mathrm{CDCl}_{3}\right) \delta 7.86(\mathrm{~d}, J=7.9 \mathrm{~Hz}, 2 \mathrm{H}), 7.33(\mathrm{~d}, J=7.9 \mathrm{~Hz}, 2 \mathrm{H}), 7.02-6.89(\mathrm{~m}, 3 \mathrm{H}), 5.25(\mathrm{~d}$, $J=9.3 \mathrm{~Hz}, 1 \mathrm{H}), 5.16-5.04(\mathrm{~m}, 2 \mathrm{H}), 4.79(\mathrm{~d}, J=9.2 \mathrm{~Hz}, 1 \mathrm{H}), 3.52$ (s, 2H), 2.45 (s, 3H), 2.30 (s, 3H). ${ }^{13} \mathrm{C} \mathrm{NMR}\left(101 \mathrm{MHz}, \mathrm{CDCl}_{3}\right) \delta 150.2,143.6,140.7,138.9,138.7,138.6,129.9,128.3$, 127.2, 125.2, 124.7, 110.9, 60.2, 36.8, 21.7, 21.5. HRMS (ESI) $m / z$ calcd for $\left[\mathrm{C}_{18} \mathrm{H}_{19} \mathrm{NO}_{2} \mathrm{SNa}\right.$, $\mathrm{M}+\mathrm{Na}]^{+}:$336.1029; found: 336.1033 .

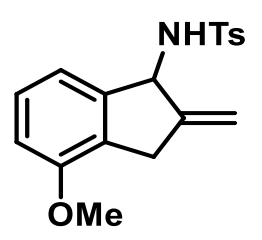

2l: petroleum ether/ethyl acetate $=3: 1(\mathrm{v} / \mathrm{v})$; yellow solid, $65.4 \mathrm{mg}, 99 \%$ yield; m.p. $171-173{ }^{\circ} \mathrm{C}$; ${ }^{1} \mathrm{H}$ NMR (400 MHz, $\left.\mathrm{CDCl}_{3}\right) \delta 7.87$ (d, $\left.J=7.8 \mathrm{~Hz}, 2 \mathrm{H}\right), 7.34$ (d, $\left.J=7.9 \mathrm{~Hz}, 2 \mathrm{H}\right), 7.14$ (t, $J=$ $7.9 \mathrm{~Hz}, 1 \mathrm{H}), 6.74-6.64(\mathrm{~m}, 2 \mathrm{H}), 5.29(\mathrm{~d}, J=9.3 \mathrm{~Hz}, 1 \mathrm{H}), 5.14(\mathrm{q}, J=2.3 \mathrm{~Hz}, 1 \mathrm{H}), 5.07$ (q, $J=$ $2.4 \mathrm{~Hz}, 1 \mathrm{H}), 4.82$ (d, $J=9.2 \mathrm{~Hz}, 1 \mathrm{H}), 3.80(\mathrm{~s}, 3 \mathrm{H}), 3.48$ (q, $J=20.5 \mathrm{~Hz}, 2 \mathrm{H}), 2.45(\mathrm{~s}, 3 \mathrm{H}) .{ }^{13} \mathrm{C}$ NMR (101 MHz, $\left.\mathrm{CDCl}_{3}\right) \delta 155.7,149.8,143.6,143.4,138.6,129.9,128.9,128.6,127.2,116.8$, 111.1, 109.7, 60.7, 55.4, 33.7, 21.7. HRMS (ESI) Calcd for $\left[\mathrm{C}_{18} \mathrm{H}_{19} \mathrm{NO}_{3} \mathrm{SNa}, \mathrm{M}+\mathrm{Na}\right]^{+}$: 352.0978; found: 352.0982 . 


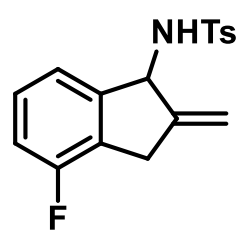

2m: petroleum ether/ethyl acetate $=5: 1(\mathrm{v} / \mathrm{v}) ;$ yellow solid, $53.0 \mathrm{mg}, 83 \%$ yield; m.p. $127-$ $129{ }^{\circ} \mathrm{C} ;{ }^{1} \mathrm{H}$ NMR $\left(400 \mathrm{MHz}, \mathrm{CDCl}_{3}\right) \delta 7.86(\mathrm{~d}, J=8.3 \mathrm{~Hz}, 2 \mathrm{H}), 7.34(\mathrm{~d}, J=7.9 \mathrm{~Hz}, 2 \mathrm{H}), 7.19$ $-7.10(\mathrm{~m}, 1 \mathrm{H}), 6.95-6.86(\mathrm{~m}, 2 \mathrm{H}), 5.30(\mathrm{~d}, J=9.3 \mathrm{~Hz}, 1 \mathrm{H}), 5.17$ (q, $J=2.4 \mathrm{~Hz}, 1 \mathrm{H}), 5.07$ (q, $J=2.4 \mathrm{~Hz}, 1 \mathrm{H}), 4.95(\mathrm{~d}, J=9.2 \mathrm{~Hz}, 1 \mathrm{H}), 3.68-3.47(\mathrm{~m}, 2 \mathrm{H}), 2.46(\mathrm{~s}, 3 \mathrm{H}) .{ }^{13} \mathrm{C} \mathrm{NMR}(101$ $\left.\mathrm{MHz}, \mathrm{CDCl}_{3}\right) \delta 160.1,157.7,148.8,145.1,145.1,143.8,138.4,130.0,129.4,129.3,127.2$, 127.0, 126.8, 120.6, 120.6, 115.1, 114.9, 111.8, 60.4, 32.9, 21.7. ${ }^{19} \mathrm{~F} \mathrm{NMR}\left(376 \mathrm{MHz}, \mathrm{CDCl}_{3}\right)$ $\delta$-118.38. HRMS (ESI) $\mathrm{m} / z$ calcd for $\left[\mathrm{C}_{17} \mathrm{H}_{15} \mathrm{FNO}_{2} \mathrm{~S}, \mathrm{M}-\mathrm{H}\right]^{-}: 316.0813$; found: 316.0810 .

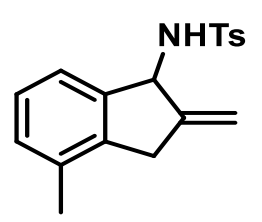

2n: petroleum ether/ethyl acetate $=5: 1(\mathrm{v} / \mathrm{v})$; yellow solid, $52.9 \mathrm{mg}, 84 \%$ yield; m.p. $133-$ $135{ }^{\circ} \mathrm{C} ;{ }^{1} \mathrm{H} \mathrm{NMR}\left(400 \mathrm{MHz}, \mathrm{CDCl}_{3}\right) \delta 7.87(\mathrm{~d}, J=8.3 \mathrm{~Hz}, 2 \mathrm{H}), 7.34(\mathrm{~d}, J=8.0 \mathrm{~Hz}, 2 \mathrm{H}), 7.06$ $(\mathrm{dt}, J=13.6,7.4 \mathrm{~Hz}, 2 \mathrm{H}), 6.88(\mathrm{~d}, J=7.3 \mathrm{~Hz}, 1 \mathrm{H}), 5.31(\mathrm{~d}, J=9.3 \mathrm{~Hz}, 1 \mathrm{H}), 5.16(\mathrm{q}, J=2.3$ $\mathrm{Hz}, 1 \mathrm{H}), 5.11$ (q, $J=2.3 \mathrm{~Hz}, 1 \mathrm{H}), 4.79(\mathrm{~d}, J=9.2 \mathrm{~Hz}, 1 \mathrm{H}), 3.48$ (d, $J=4.3 \mathrm{~Hz}, 2 \mathrm{H}), 2.46$ (s, $3 \mathrm{H}), 2.21(\mathrm{~s}, 3 \mathrm{H}) .{ }^{13} \mathrm{C} \mathrm{NMR}\left(101 \mathrm{MHz}, \mathrm{CDCl}_{3}\right) \delta 149.8,143.6,141.5,139.4,138.6,134.1$, 129.9, 129.3, 127.6, 127.2, 122.1, 111.1, 60.7, 35.7, 21.7, 18.8. HRMS (ESI) Calcd for $\left[\mathrm{C}_{18} \mathrm{H}_{18} \mathrm{NO}_{2} \mathrm{~S}, \mathrm{M}-\mathrm{H}\right]^{-}:$312.1064; found: 312.1060.

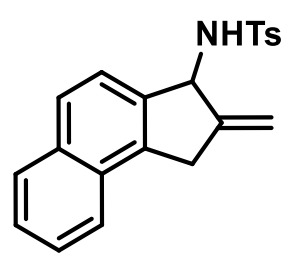

2o: petroleum ether/ethyl acetate $=5: 1(\mathrm{v} / \mathrm{v})$; yellow solid, $63.4 \mathrm{mg}, 91 \%$ yield; m.p. $181-$ $183{ }^{\circ} \mathrm{C} ;{ }^{1} \mathrm{H}$ NMR $\left(400 \mathrm{MHz}, \mathrm{CDCl}_{3}\right) \delta 7.91(\mathrm{~d}, J=7.9 \mathrm{~Hz}, 2 \mathrm{H}), 7.84(\mathrm{~d}, J=7.5 \mathrm{~Hz}, 1 \mathrm{H}), 7.70$ $(\mathrm{dd}, J=12.0,8.2 \mathrm{~Hz}, 2 \mathrm{H}), 7.56-7.45(\mathrm{~m}, 2 \mathrm{H}), 7.36(\mathrm{~d}, J=7.9 \mathrm{~Hz}, 2 \mathrm{H}), 7.25(\mathrm{~d}, J=4.7 \mathrm{~Hz}$, $1 \mathrm{H}), 5.48(\mathrm{~d}, J=9.3 \mathrm{~Hz}, 1 \mathrm{H}), 5.26(\mathrm{q}, J=2.3 \mathrm{~Hz}, 1 \mathrm{H}), 5.12(\mathrm{q}, J=2.4 \mathrm{~Hz}, 1 \mathrm{H}), 4.86(\mathrm{~d}, J=$ $9.2 \mathrm{~Hz}, 1 \mathrm{H}), 3.98-3.76(\mathrm{~m}, 2 \mathrm{H}), 2.47$ (s, 3H). ${ }^{13} \mathrm{C} \mathrm{NMR}\left(101 \mathrm{MHz}, \mathrm{CDCl}_{3}\right) \delta 149.7,143.7$, 138.7, 138.4, 137.0, 133.7, 130.0, 129.8, 128.8, 128.4, 127.3, 126.6, 126.2, 124.3, 122.6, 111.8, 61.3, 35.4, 21.7. HRMS (ESI) Calcd for $\left[\mathrm{C}_{21} \mathrm{H}_{18} \mathrm{NO}_{2} \mathrm{~S}, \mathrm{M}-\mathrm{H}\right]^{-}: 348.1064$; found: 348.1061 . 


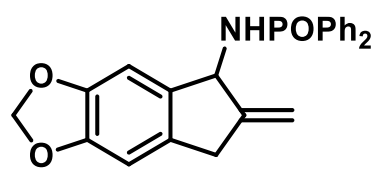

2p: petroleum ether/ethyl acetate $=1: 2(\mathrm{v} / \mathrm{v})$; pale orange solid, $64.3 \mathrm{mg}, 83 \%$ yield; m.p. $147-$ $149{ }^{\circ} \mathrm{C} ;{ }^{1} \mathrm{H}$ NMR $\left(400 \mathrm{MHz}, \mathrm{CDCl}_{3}\right) \delta 8.09-7.97(\mathrm{~m}, 4 \mathrm{H}), 7.60-7.40(\mathrm{~m}, 6 \mathrm{H}), 7.34(\mathrm{~s}, 1 \mathrm{H})$, $6.62(\mathrm{~s}, 1 \mathrm{H}), 5.93(\mathrm{~d}, J=8.2,1.4 \mathrm{~Hz}, 2 \mathrm{H}), 5.69(\mathrm{~s}, 1 \mathrm{H}), 5.23(\mathrm{~s}, 1 \mathrm{H}), 4.93(\mathrm{t}, J=10.4 \mathrm{~Hz}, 1 \mathrm{H})$, 3.49 (s, 2H), 3.26-3.16 (m, 1H).; ${ }^{13} \mathrm{C}$ NMR (101 MHz, $\left.\mathrm{CDCl}_{3}\right) \delta 152.7,152.6,147.9,147.3$, 136.9, 133.4, 133.3, 132.6, 132.5, 132.3, 132.2, 132.1, 132.1, 128.8, 128.7, 110.1, 106.1, 104.6, 101.1, 58.8, 37.0. ${ }^{31} \mathrm{P}$ NMR $\left(162 \mathrm{MHz}, \mathrm{CDCl}_{3}\right) \delta 22.14$. HRMS (ESI) Calcd for $\left[\mathrm{C}_{23} \mathrm{H}_{19} \mathrm{NO}_{3} \mathrm{P}\right.$, $\mathrm{M}-\mathrm{H}]^{-}:$388.1108; found: 388.1218 .

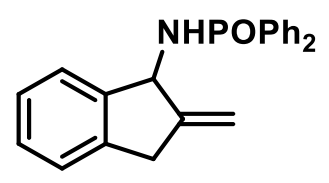

2q: petro ether/ethyl acetate $=1: 1(\mathrm{v} / \mathrm{v})$; white solid, $67.6 \mathrm{mg}, 98 \%$ yield; $1.66 \mathrm{~g}, 96 \%$ yield $(5.0$ mmol scale); m.p. $185-186{ }^{\circ} \mathrm{C} ;{ }^{1} \mathrm{H}$ NMR $\left(400 \mathrm{MHz}, \mathrm{CDCl}_{3}\right) \delta 8.08(\mathrm{dd}, J=12.0,7.2 \mathrm{~Hz}, 4 \mathrm{H})$, $7.87(\mathrm{~d}, J=7.4 \mathrm{~Hz}, 1 \mathrm{H}), 7.60-7.45(\mathrm{~m}, 6 \mathrm{H}), 7.34-7.16(\mathrm{~m}, 3 \mathrm{H}), 5.77$ (d, $J=2.5 \mathrm{~Hz}, 1 \mathrm{H}), 5.30$ $(\mathrm{d}, J=2.5 \mathrm{~Hz}, 1 \mathrm{H}), 5.06(\mathrm{t}, J=10.5 \mathrm{~Hz}, 1 \mathrm{H}), 3.61(\mathrm{~s}, 2 \mathrm{H}), 3.29(\mathrm{dd}, J=11.6,6.4 \mathrm{~Hz}, 1 \mathrm{H}) .{ }^{13} \mathrm{C}$ $\operatorname{NMR}\left(101 \mathrm{MHz}, \mathrm{CDCl}_{3}\right) \delta 152.5,152.5,144.1,140.3,133.5,133.0,132.6,132.5,132.4,132.3$, 132.2, 132.1, 132.1, 131.7, 128.8, 128.7, 128.6, 128.1, 127.4, 125.6, 124.4, 110.1, 58.9, 37.1. ${ }^{31} \mathrm{P}$ NMR $\left(162 \mathrm{MHz}, \mathrm{CDCl}_{3}\right) \delta 22.25$. HRMS (ESI) $\mathrm{m} / z$ Calcd for $\left[\mathrm{C}_{22} \mathrm{H}_{20} \mathrm{NOPNa}, \mathrm{M}+\mathrm{Na}\right]^{+}$: 368.1175; found: 368.1179 .

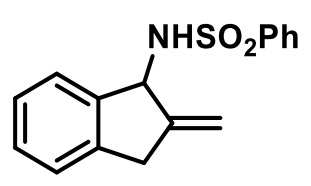

2s: petroleum ether/ethyl acetate $=5: 1(\mathrm{v} / \mathrm{v})$; pale yellow solid, $50.3 \mathrm{mg}$, 88\% yield; m.p. 93$95{ }^{\circ} \mathrm{C} ;{ }^{1} \mathrm{H}$ NMR $\left(400 \mathrm{MHz}, \mathrm{CDCl}_{3}\right) \delta 8.02-7.96(\mathrm{~m}, 2 \mathrm{H}), 7.65-7.50(\mathrm{~m}, 3 \mathrm{H}), 7.24-7.10(\mathrm{~m}$, $3 \mathrm{H}), 7.02(\mathrm{~d}, J=7.6 \mathrm{~Hz}, 1 \mathrm{H}), 5.32(\mathrm{~d}, J=9.3 \mathrm{~Hz}, 1 \mathrm{H}), 5.12(\mathrm{q}, J=2.3 \mathrm{~Hz}, 1 \mathrm{H}), 5.06$ (q, $J=$ $2.4 \mathrm{~Hz}, 1 \mathrm{H}), 4.95(\mathrm{~d}, J=9.3 \mathrm{~Hz}, 1 \mathrm{H}), 3.57(\mathrm{~s}, 2 \mathrm{H}) .{ }^{13} \mathrm{C} \mathrm{NMR}\left(101 \mathrm{MHz}, \mathrm{CDCl}_{3}\right) \delta 149.7,141.6$, 141.6, 140.5, 132.8, 129.4, 128.7, 127.4, 127.1, 124.9, 124.7, 111.0, 60.5, 36.8. HRMS (ESI) $m / z$ calcd for $\left[\mathrm{C}_{16} \mathrm{H}_{15} \mathrm{NO}_{2} \mathrm{SNa}, \mathrm{M}+\mathrm{Na}\right]^{+}: 308.0716$; found: 308.0720 . 


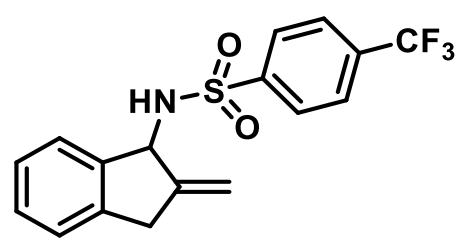

2r: petroleum ether/ethyl acetate $=5: 1(\mathrm{v} / \mathrm{v})$; pale yellow solid, $56.9 \mathrm{mg}, 80 \%$ yield; m.p. 145$147{ }^{\circ} \mathrm{C} ;{ }^{1} \mathrm{H}$ NMR $\left(400 \mathrm{MHz}, \mathrm{CDCl}_{3}\right) \delta 8.15(\mathrm{~d}, J=8.1 \mathrm{~Hz}, 2 \mathrm{H}), 7.87(\mathrm{~d}, J=8.1 \mathrm{~Hz}, 2 \mathrm{H}), 7.36$ - $7.06(\mathrm{~m}, 4 \mathrm{H}), 5.40(\mathrm{~d}, J=9.3 \mathrm{~Hz}, 1 \mathrm{H}), 5.27-4.95(\mathrm{~m}, 3 \mathrm{H}), 3.63(\mathrm{~s}, 2 \mathrm{H}) .{ }^{13} \mathrm{C} \mathrm{NMR}(101$ $\left.\mathrm{MHz}, \mathrm{CDCl}_{3}\right) \delta 149.4,145.2,141.1,140.6,134.7,134.4,128.9,127.6,127.5,126.5$ (q, $J_{\mathrm{C}, \mathrm{F}}=$ 3.6 Hz), 124.9, 124.8, 111.2, 60.7, 36.8. $\left.{ }^{19} \mathrm{~F} \mathrm{NMR} \mathrm{(376} \mathrm{MHz,} \mathrm{CDCl}_{3}\right) \delta-63.04$. HRMS (ESI) $m / z$ calcd for $\left[\mathrm{C}_{17} \mathrm{H}_{14} \mathrm{~F}_{3} \mathrm{NO}_{2} \mathrm{SNa}, \mathrm{M}+\mathrm{Na}\right]^{+}$: 376.0590 ; found: 376.0594 .

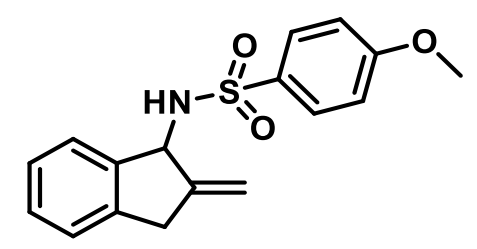

2t: petroleum ether/ethyl acetate $=3: 1(\mathrm{v} / \mathrm{v})$; pale orange oil, $52.7 \mathrm{mg}, 84 \%$ yield; ${ }^{1} \mathrm{H}$ NMR $\left(400 \mathrm{MHz}, \mathrm{CDCl}_{3}\right) \delta$ 7.95-7.89 (m, 2H), 7.25-7.08 (m, 4H), 7.04-6.98 (m, 2H), 5.29 (d, $J=$ $9.0 \mathrm{~Hz}, 1 \mathrm{H}), 5.17-5.06(\mathrm{~m}, 2 \mathrm{H}), 4.86(\mathrm{~d}, J=9.2 \mathrm{~Hz}, 1 \mathrm{H}), 3.89(\mathrm{~s}, 3 \mathrm{H}), 3.58(\mathrm{~s}, 2 \mathrm{H}) .{ }^{13} \mathrm{C} \mathrm{NMR}$ $\left(101 \mathrm{MHz}, \mathrm{CDCl}_{3}\right) \delta 163.1,149.9,141.8,140.5,133.2,129.3,128.6,127.4,124.9,124.7,114.5$, 110.9, 60.3, 55.8, 36.9. HRMS (ESI) Calcd for $\left[\mathrm{C}_{17} \mathrm{H}_{16} \mathrm{NO}_{3} \mathrm{~S}, \mathrm{M}-\mathrm{H}\right]^{-}$: 314.0856; found: 314.0852 .

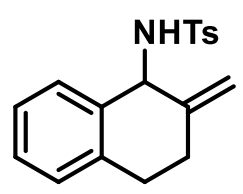

5a: petroleum ether/ethyl acetate $=5: 1(\mathrm{v} / \mathrm{v})$; white solid, $59.3 \mathrm{mg}, 95 \%$ yield; m.p. $125-127{ }^{\circ} \mathrm{C}$; ${ }^{1} \mathrm{H}$ NMR (400 MHz, $\left.\mathrm{CDCl}_{3}\right) \delta 7.80-7.72(\mathrm{~m}, 2 \mathrm{H}), 7.28(\mathrm{~d}, J=8.0 \mathrm{~Hz}, 2 \mathrm{H}), 7.20-7.10(\mathrm{~m}, 3 \mathrm{H})$, 7.07-7.00 (m, 1H), 4.97 (d, J=7.4 Hz, 1H), 4.91-4.81 (m, 3H), 2.96-2.68 (m, 2H), 2.50-2.24 $(\mathrm{m}, 5 \mathrm{H}) .{ }^{13} \mathrm{C} \mathrm{NMR}\left(101 \mathrm{MHz}, \mathrm{CDCl}_{3}\right) \delta 144.3,143.3,138.3,137.4,136.0,129.6,128.9,128.8$, 127.9, 127.3, 126.8, 111.7, 57.1, 31.0, 29.0, 21.7. HRMS (ESI) Calcd for $\left[\mathrm{C}_{18} \mathrm{H}_{18} \mathrm{NO}_{2} \mathrm{~S}, \mathrm{M}-\mathrm{H}\right]^{-}$: 312.1064; found: 312.1060 . 


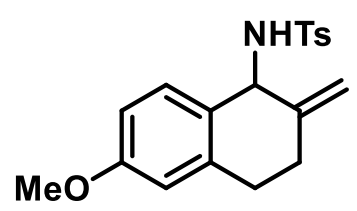

5b: petroleum ether/ethyl acetate $=3: 1(\mathrm{v} / \mathrm{v})$; pale yellow solid, $66.4 \mathrm{mg} ; 97 \%$ yield; m.p. $127-$ $129{ }^{\circ} \mathrm{C} ;{ }^{1} \mathrm{H}$ NMR $\left(400 \mathrm{MHz}, \mathrm{CDCl}_{3}\right) \delta$ 7.78-7.73 (m, 2H), 7.31-7.24 (m, 2H), $7.07(\mathrm{~d}, J=8.6$ $\mathrm{Hz}, 1 \mathrm{H}), 6.69$ (dd, $J=8.6,2.7 \mathrm{~Hz}, 1 \mathrm{H}), 6.55$ (d, $J=2.7 \mathrm{~Hz}, 1 \mathrm{H}), 4.96-4.78$ (m, 4H), 3.75 (s, $3 \mathrm{H}), 2.88-2.66$ (m, 2H), 2.47-2.23 (m, 5H). ${ }^{13} \mathrm{C} \mathrm{NMR}\left(101 \mathrm{MHz}, \mathrm{CDCl}_{3}\right) \delta 159.1,144.4,143.3$, 138.8, 138.4, 130.2, 129.6, 128.2, 127.3, 113.2, 113.1, 111.7, 56.7, 55.3, 31.3, 28.8, 21.7. HRMS (ESI) $m / z$ Calcd for $\left[\mathrm{C}_{19} \mathrm{H}_{20} \mathrm{NO}_{3} \mathrm{~S}, \mathrm{M}-\mathrm{H}\right]^{-}: 342.1169$; found: 342.1166.<smiles>C=C1CCc2cc(F)ccc2C1NC(N)=S</smiles>

5c: petroleum ether/ethyl acetate $=5: 1(\mathrm{v} / \mathrm{v})$; pale yellow solid, $61.5 \mathrm{mg}$, 93\% yield; m.p. $143-$ $145{ }^{\circ} \mathrm{C} ;{ }^{1} \mathrm{H}$ NMR $\left(400 \mathrm{MHz}, \mathrm{CDCl}_{3}\right) \delta 7.75(\mathrm{~d}, J=8.1 \mathrm{~Hz}, 2 \mathrm{H}), 7.28(\mathrm{~d}, J=8.0 \mathrm{~Hz}, 2 \mathrm{H}), 7.23$ - $7.16(\mathrm{~m}, 1 \mathrm{H}), 6.84(\mathrm{td}, J=8.5,2.7 \mathrm{~Hz}, 1 \mathrm{H}), 6.76-6.70(\mathrm{~m}, 1 \mathrm{H}), 4.98-4.79(\mathrm{~m}, 4 \mathrm{H}), 2.89$ $2.68(\mathrm{~m}, 2 \mathrm{H}), 2.47-2.26(\mathrm{~m}, 5 \mathrm{H}) .{ }^{13} \mathrm{C} \mathrm{NMR}\left(101 \mathrm{MHz}, \mathrm{CDCl}_{3}\right) \delta 163.4,160.9,143.8,143.5$, 139.8, 139.7, 138.3, 131.9, 131.8, 130.9, 130.8, 129.8, 129.7, 127.3, 115.1, 114.9, 114.2, 114.0, 112.0, 56.5, 31.0, 28.7, 21.7. ${ }^{19} \mathrm{~F}$ NMR $\left(376 \mathrm{MHz} \mathrm{CDCl}_{3}\right) \delta-114.51$. HRMS (ESI) m/z Calcd for $\left[\mathrm{C}_{18} \mathrm{H}_{17} \mathrm{FNO}_{2} \mathrm{~S}, \mathrm{M}-\mathrm{H}\right]^{-}:$330.0970; found: 330.0966 .<smiles>C=C1CCc2cccc(C)c2C1NC(F)(F)F</smiles>

5d: petroleum ether/ethyl acetate $=5: 1(\mathrm{v} / \mathrm{v})$; white solid, $61.4 \mathrm{mg}, 94 \%$ yield; m.p. $125-127{ }^{\circ} \mathrm{C}$; ${ }^{1} \mathrm{H}$ NMR (400 MHz, $\left.\mathrm{CDCl}_{3}\right) \delta 7.73(\mathrm{~d}, J=8.0 \mathrm{~Hz}, 2 \mathrm{H}), 7.27$ (d, $\left.J=8.0 \mathrm{~Hz}, 2 \mathrm{H}\right), 7.17-7.05$ (m, 2H), $6.94(\mathrm{~d}, J=7.6 \mathrm{~Hz}, 1 \mathrm{H}), 5.09$ (d, $J=5.8 \mathrm{~Hz}, 1 \mathrm{H}), 5.03-4.99(\mathrm{~m}, 1 \mathrm{H}), 4.81-4.75(\mathrm{~m}, 1 \mathrm{H})$, $4.56(\mathrm{~d}, J=6.0 \mathrm{~Hz}, 1 \mathrm{H}), 3.04-2.69(\mathrm{~m}, 2 \mathrm{H}), 2.50-2.38(\mathrm{~m}, 6 \mathrm{H}), 2.36-2.08(\mathrm{~m}, 2 \mathrm{H}) .{ }^{13} \mathrm{C} \mathrm{NMR}$ $\left(101 \mathrm{MHz}, \mathrm{CDCl}_{3}\right) \delta 143.5,143.0,138.8,137.6,137.4,133.5,129.3,129.0,128.3,127.4,127.0$, 113.0, 55.5, 31.3, 26.5, 21.7, 18.7. HRMS (ESI) $\mathrm{m} / \mathrm{z}$ Calcd for $\left[\mathrm{C}_{19} \mathrm{H}_{20} \mathrm{NO}_{2} \mathrm{~S}, \mathrm{M}-\mathrm{H}\right]^{-}: 326.1220$; found: 326.1218 . 


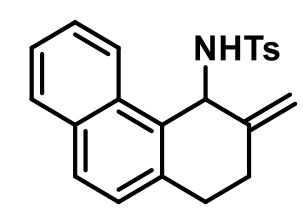

5e: petroleum ether/ethyl acetate $=5: 1(\mathrm{v} / \mathrm{v})$; yellow solid, $69.5 \mathrm{mg}, 96 \%$ yield; m.p. 183$185{ }^{\circ} \mathrm{C} ;{ }^{1} \mathrm{H}$ NMR (400 MHz, $\left.\mathrm{CDCl}_{3}\right) \delta 8.04(\mathrm{~d}, J=8.4 \mathrm{~Hz}, 1 \mathrm{H}), 7.81-7.66(\mathrm{~m}, 4 \mathrm{H}), 7.54-7.43$ (m, 2H), 7.31-7.24 (m, 2H), $7.14(\mathrm{~d}, J=8.5 \mathrm{~Hz}, 1 \mathrm{H}), 5.57$ (d, $J=5.1 \mathrm{~Hz}, 1 \mathrm{H}), 5.10-5.06$ (m, $1 \mathrm{H}), 4.85-4.81(\mathrm{~m}, 1 \mathrm{H}), 4.72(\mathrm{~d}, J=5.1 \mathrm{~Hz}, 1 \mathrm{H}), 3.09-2.86(\mathrm{~m}, 2 \mathrm{H}), 2.50-2.38(\mathrm{~m}, 4 \mathrm{H}), 2.25-$ $2.16(\mathrm{~m}, 1 \mathrm{H}) .{ }^{13} \mathrm{C} \mathrm{NMR}\left(101 \mathrm{MHz}, \mathrm{CDCl}_{3}\right) \delta 143.1,142.9,138.5,135.6,132.7,131.2,129.2$, 129.0, 128.9, 128.6, 127.5, 127.4, 127.2, 125.5, 123.0, 113.0, 54.5, 32.0, 26.4, 21.6. HRMS (ESI) $m / z$ Calcd for $\left[\mathrm{C}_{22} \mathrm{H}_{21} \mathrm{NO}_{2} \mathrm{SNa}, \mathrm{M}+\mathrm{Na}\right]^{+}: 386.1185$; found: 386.1188 .

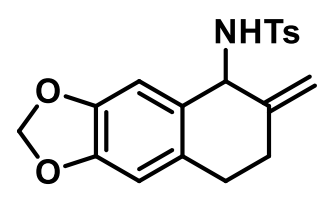

5f: petroleum ether/ethyl acetate $=3: 1(\mathrm{v} / \mathrm{v})$; yellow solid, $67.7 \mathrm{mg}, 95 \%$ yield; m.p. $153-155^{\circ} \mathrm{C}$; ${ }^{1} \mathrm{H}$ NMR (400 MHz, $\left.\mathrm{CDCl}_{3}\right) \delta 7.80$ - $7.73(\mathrm{~m}, 2 \mathrm{H}), 7.35$ - $7.27(\mathrm{~m}, 2 \mathrm{H}), 6.61(\mathrm{~s}, 1 \mathrm{H}), 6.47$ (s, $1 \mathrm{H}), 5.90-5.86(\mathrm{~m}, 2 \mathrm{H}), 4.91-4.73(\mathrm{~m}, 4 \mathrm{H}), 2.80-2.70(\mathrm{~m}, 1 \mathrm{H}), 2.70-2.60(\mathrm{~m}, 1 \mathrm{H}), 2.47$ $-2.32(\mathrm{~m}, 4 \mathrm{H}), 2.32-2.23(\mathrm{~m}, 1 \mathrm{H}) .{ }^{13} \mathrm{C} \mathrm{NMR}\left(101 \mathrm{MHz}, \mathrm{CDCl}_{3}\right) \delta 147.5,146.6,144.2,143.4$, 138.4, 131.0, 129.6, 128.8, 127.4, 111.7, 108.6, 108.3, 101.1, 57.2, 31.1, 28.9, 21.7. HRMS (ESI) $\mathrm{m} / \mathrm{z}$ Calcd for $\left[\mathrm{C}_{19} \mathrm{H}_{18} \mathrm{NO}_{4} \mathrm{~S}, \mathrm{M}-\mathrm{H}\right]^{-}: 356.0962$; found: 356.0960 .

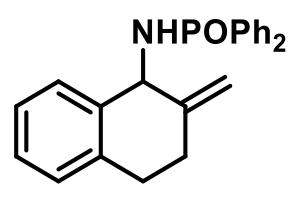

5g: petro ether/ethyl acetate $=1: 3(\mathrm{v} / \mathrm{v})$ for flash chromatography; white solid, $49.5 \mathrm{mg}, 70 \%$ yield; m.p. $177-179{ }^{\circ} \mathrm{C} ;{ }^{1} \mathrm{H}$ NMR (400 MHz, $\left.\mathrm{CDCl}_{3}\right) \delta 8.04-7.91(\mathrm{~m}, 4 \mathrm{H}), 7.83(\mathrm{~d}, J=7.8 \mathrm{~Hz}$, 1H), 7.52-7.40 (m, 6H), 7.29-7.23 (m, 1H), 7.19-7.13 (m, 1H), 7.05 (d, J = 7.5 Hz, 1H), 4.99 $(\mathrm{s}, 1 \mathrm{H}), 4.93(\mathrm{~s}, 1 \mathrm{H}), 4.80(\mathrm{t}, J=9.9 \mathrm{~Hz}, 1 \mathrm{H}), 3.34-3.24(\mathrm{~m}, 1 \mathrm{H}), 2.95-2.75(\mathrm{~m}, 2 \mathrm{H}), 2.67-2.38$ $(\mathrm{m}, 2 \mathrm{H}) .{ }^{13} \mathrm{C} \mathrm{NMR}\left(101 \mathrm{MHz}, \mathrm{CDCl}_{3}\right) \delta 147.1,147.0,138.9,138.8,137.1,132.8,132.7,132.1$, 132.0, 129.2, 128.7, 128.6, 128.5, 128.5, 128.4, 127.4, 126.9, 110.3, 54.6, 31.3, 29.6. ${ }^{31} \mathrm{P}$ NMR $\left(162 \mathrm{MHz} \mathrm{CDCl}_{3}\right) \delta$ 22.09. HRMS (ESI) $\mathrm{m} / z$ Calcd for $\left[\mathrm{C}_{23} \mathrm{H}_{22} \mathrm{NOPNa}, \mathrm{M}+\mathrm{Na}\right]^{+}: 382.1331$; found: 382.1336 . 


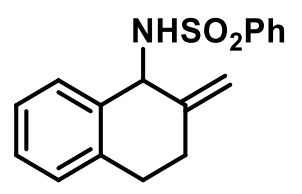

5h: petroleum ether/ethyl acetate $=5: 1(\mathrm{v} / \mathrm{v})$; white solid, $59.7 \mathrm{mg}, 99 \%$ yield; ${ }^{1} \mathrm{H}$ NMR $(400$ $\left.\mathrm{MHz}, \mathrm{CDCl}_{3}\right) \delta$ 7.91-7.84 (m, 2H), 7.60-7.45 (m, 3H), 7.21-7.09 (m, 3H), 7.06-6.99 (m, 1H), 5.05-4.96 (m, 2H), 4.86-4.77 (m, 2H), 2.91-2.68 (m, 2H), 2.46-2.25 (m, 2H). ${ }^{13} \mathrm{C}$ NMR (101 $\left.\mathrm{MHz}, \mathrm{CDCl}_{3}\right) \delta 144.1,141.3,137.4,135.9,132.6,129.0,128.9,128.8,127.9,127.2,126.8$, 111.7, 57.1, 31.0, 28.9. HRMS (ESI) $\mathrm{m} / z$ Calcd for $\left[\mathrm{C}_{17} \mathrm{H}_{17} \mathrm{NO}_{2} \mathrm{SNa}, \mathrm{M}+\mathrm{Na}\right]^{+}$: 322.0872; found: 322.0873 .

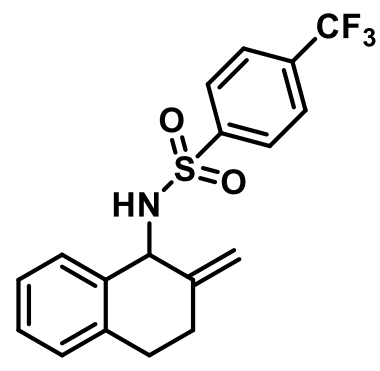

5j: petroleum ether/ethyl acetate $=5: 1(\mathrm{v} / \mathrm{v})$; white solid, $64.9 \mathrm{mg}, 88 \%$ yield; ${ }^{1} \mathrm{H}$ NMR $(400$ $\left.\mathrm{MHz}, \mathrm{CDCl}_{3}\right) \delta 7.95(\mathrm{~d}, J=8.2 \mathrm{~Hz}, 2 \mathrm{H}), 7.72(\mathrm{~d}, J=8.2 \mathrm{~Hz}, 2 \mathrm{H}), 7.30-7.10(\mathrm{~m}, 3 \mathrm{H}), 7.06-$ $6.99(\mathrm{~m}, 1 \mathrm{H}), 5.25(\mathrm{~d}, J=7.5 \mathrm{~Hz}, 1 \mathrm{H}), 5.03(\mathrm{~d}, J=7.7 \mathrm{~Hz}, 1 \mathrm{H}), 4.85-4.77(\mathrm{~m}, 2 \mathrm{H}), 2.90-$ $2.67(\mathrm{~m}, 2 \mathrm{H}), 2.43-2.21(\mathrm{~m}, 2 \mathrm{H}) .{ }^{13} \mathrm{C} \mathrm{NMR}\left(101 \mathrm{MHz}, \mathrm{CDCl}_{3}\right) \delta 145.0,143.8,137.4,135.4$, 129.0, 128.9, 128.2, 127.7, 126.9, 126.1 (q, $\left.J_{\mathrm{C}, \mathrm{F}}=3.4 \mathrm{~Hz}\right), 112.1,57.4,30.9,28.7 .{ }^{19} \mathrm{~F} \mathrm{NMR}$ $\left(376 \mathrm{MHz}, \mathrm{CDCl}_{3}\right) \delta$-63.05. HRMS (ESI) $\mathrm{m} / \mathrm{z}$ Calcd for $\left[\mathrm{C}_{18} \mathrm{H}_{16} \mathrm{~F}_{3} \mathrm{NO}_{2} \mathrm{SNa}, \mathrm{M}+\mathrm{Na}\right]^{+}$: 390.0746; found: 390.0750 .

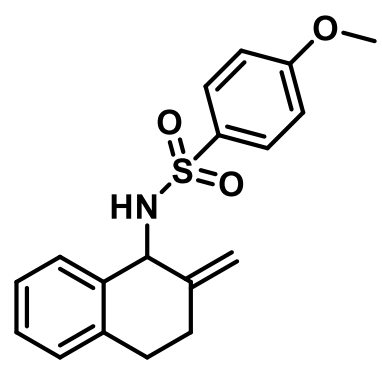

5i: petroleum ether/ethyl acetate $=3: 1(\mathrm{v} / \mathrm{v})$; white solid, $64.3 \mathrm{mg}$, 98\% yield; ${ }^{1} \mathrm{H}$ NMR (400 $\left.\mathrm{MHz} \mathrm{CDCl}_{3}\right) \delta 7.88-7.77(\mathrm{~m}, 2 \mathrm{H}), 7.24-7.12(\mathrm{~m}, 3 \mathrm{H}), 7.08-7.02(\mathrm{~m}, 1 \mathrm{H}), 7.01-6.92(\mathrm{~m}$, $2 \mathrm{H}), 5.06-4.74(\mathrm{~m}, 4 \mathrm{H}), 3.87(\mathrm{~s}, 3 \mathrm{H}), 2.94-2.68(\mathrm{~m}, 2 \mathrm{H}), 2.50-2.25(\mathrm{~m}, 2 \mathrm{H}) .{ }^{13} \mathrm{C} \mathrm{NMR}$ $\left(101 \mathrm{MHz}, \mathrm{CDCl}_{3}\right) \delta 162.8,144.3,137.4,136.0,133.0,129.5,128.9,128.8,127.9,126.8,114.1$, 111.7, 57.0, 55.7, 31.0, 29.0. HRMS (ESI) $\mathrm{m} / z$ Calcd for $\left[\mathrm{C}_{18} \mathrm{H}_{19} \mathrm{NO}_{3} \mathrm{SNa}, \mathrm{M}+\mathrm{Na}\right]^{+}: 352.0978$; 
found: 352.0983 . 


\section{HPLC Charts}

Condition : Chiralpak OJ-3 column, $n$-hexane $/ i-\mathrm{PrOH}=90: 10$, flow rate $=1.0 \mathrm{~mL} / \mathrm{min}, 230$ nm UV detector

HPLC Chart of Product 2a using $(R, R)-\mathrm{BDPP}(\mathbf{L 1})$
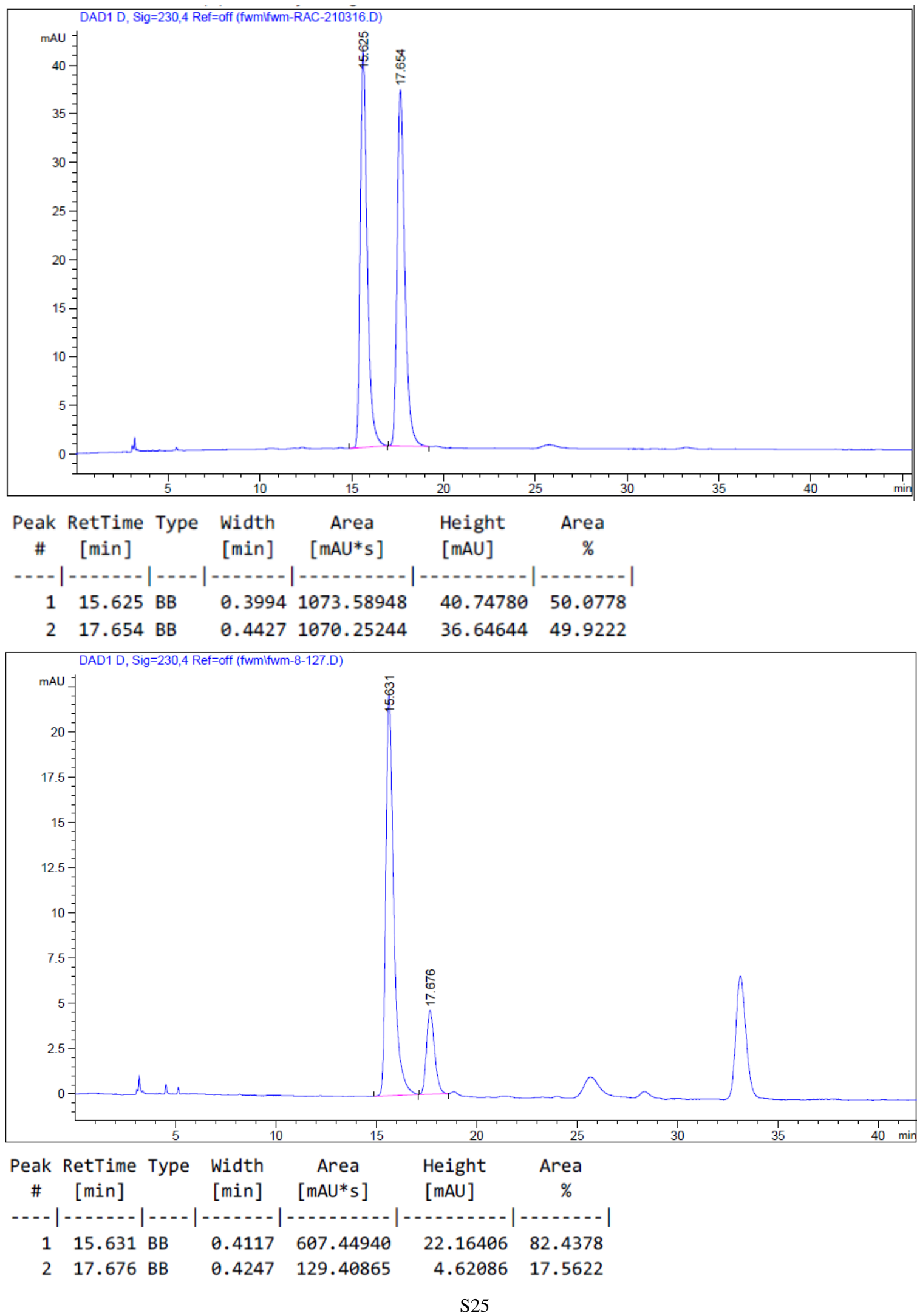
HPLC Chart of Product 2a using SITCP L2
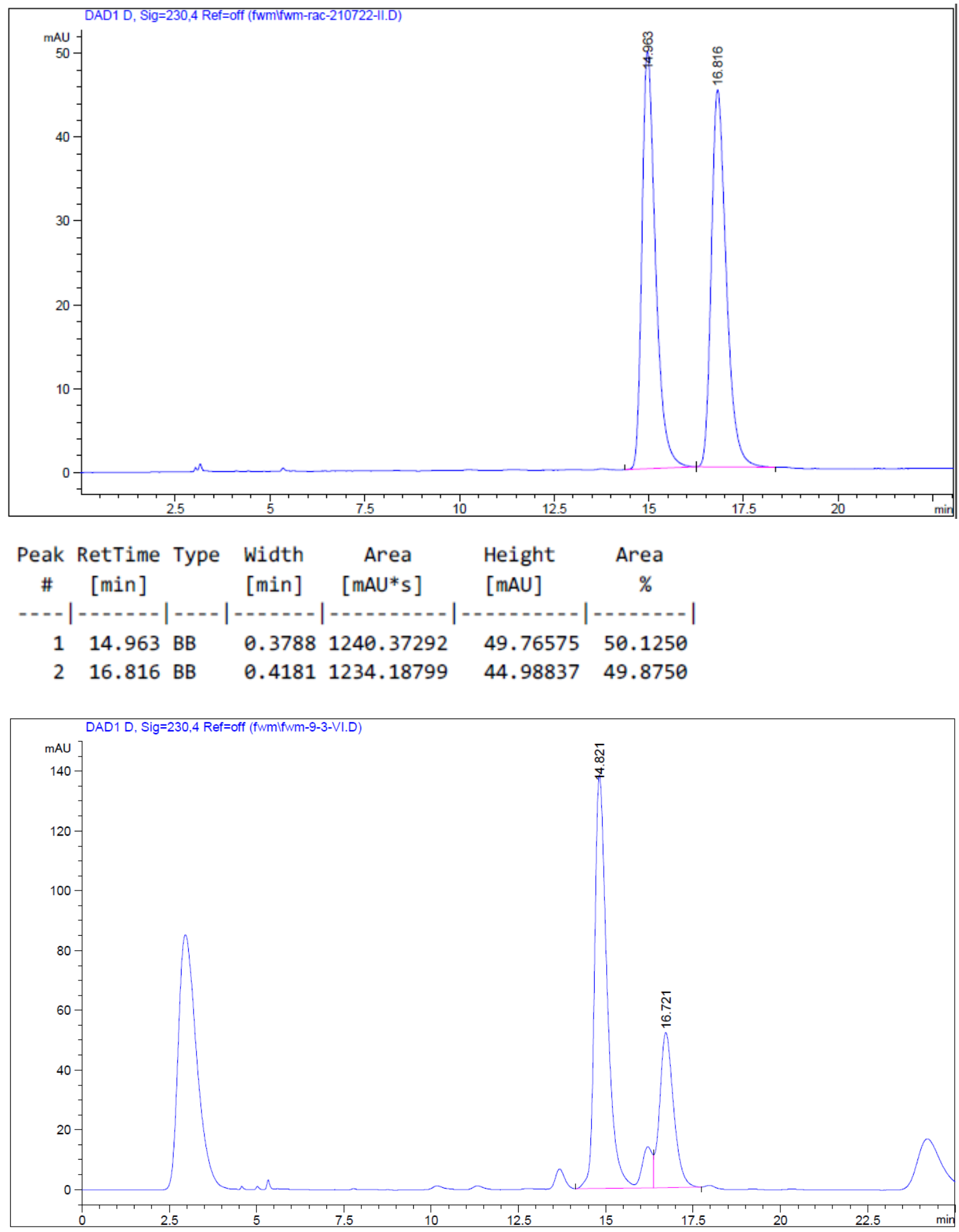

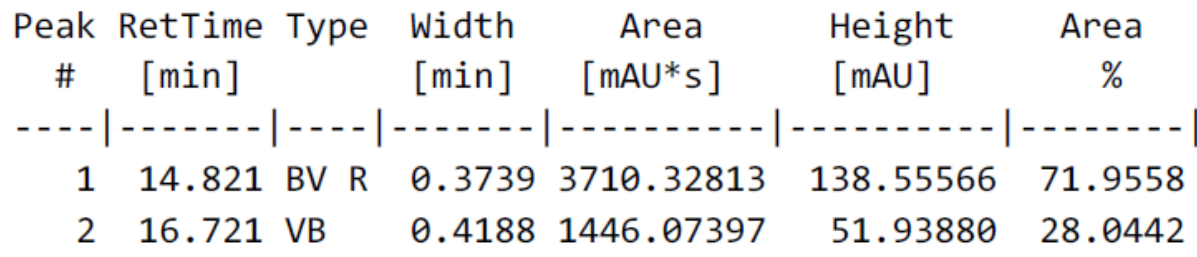


HPLC Chart of Product 2a using $\mathbf{L 3}$
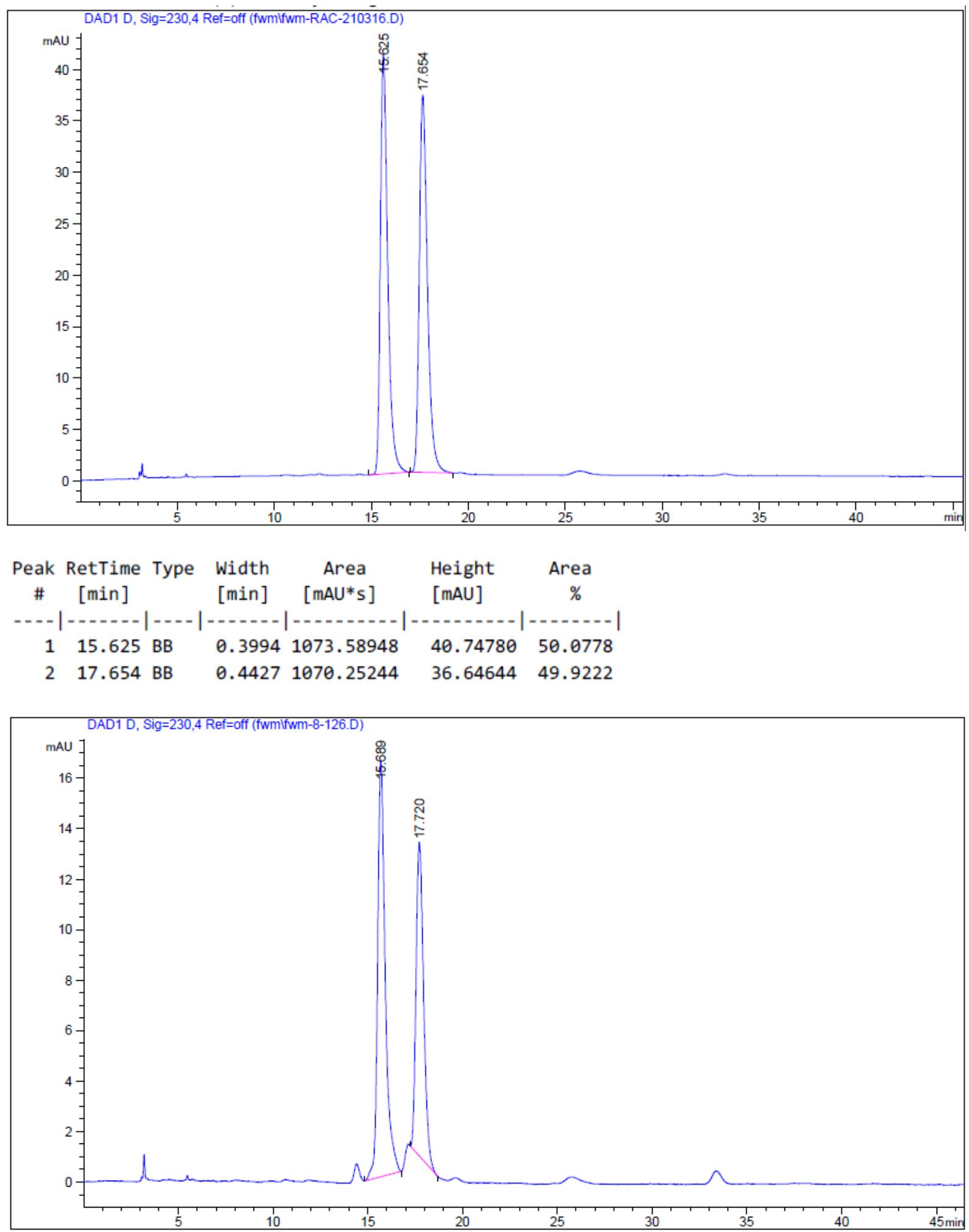

\begin{tabular}{|c|c|c|c|c|c|c|}
\hline $\begin{array}{c}\text { eak } \\
\#\end{array}$ & $\begin{array}{c}\text { RetTime } \\
\text { [min] }\end{array}$ & Type & $\begin{array}{l}\text { Width } \\
\text { [min] }\end{array}$ & $\begin{array}{c}\text { Area } \\
{\left[\mathrm{mAU}^{*} \mathrm{~s}\right]}\end{array}$ & $\begin{array}{l}\text { Height } \\
\text { [mAU] }\end{array}$ & $\begin{array}{c}\text { Area } \\
\%\end{array}$ \\
\hline & & & & & & \\
\hline 1 & & & & 459. & 16 & 57. \\
\hline 2 & 17. & BB & 286 & 343.56537 & 12.42350 & 42.7552 \\
\hline
\end{tabular}


HPLC Chart of Product 2a using (S,S)-DIOP (L4)
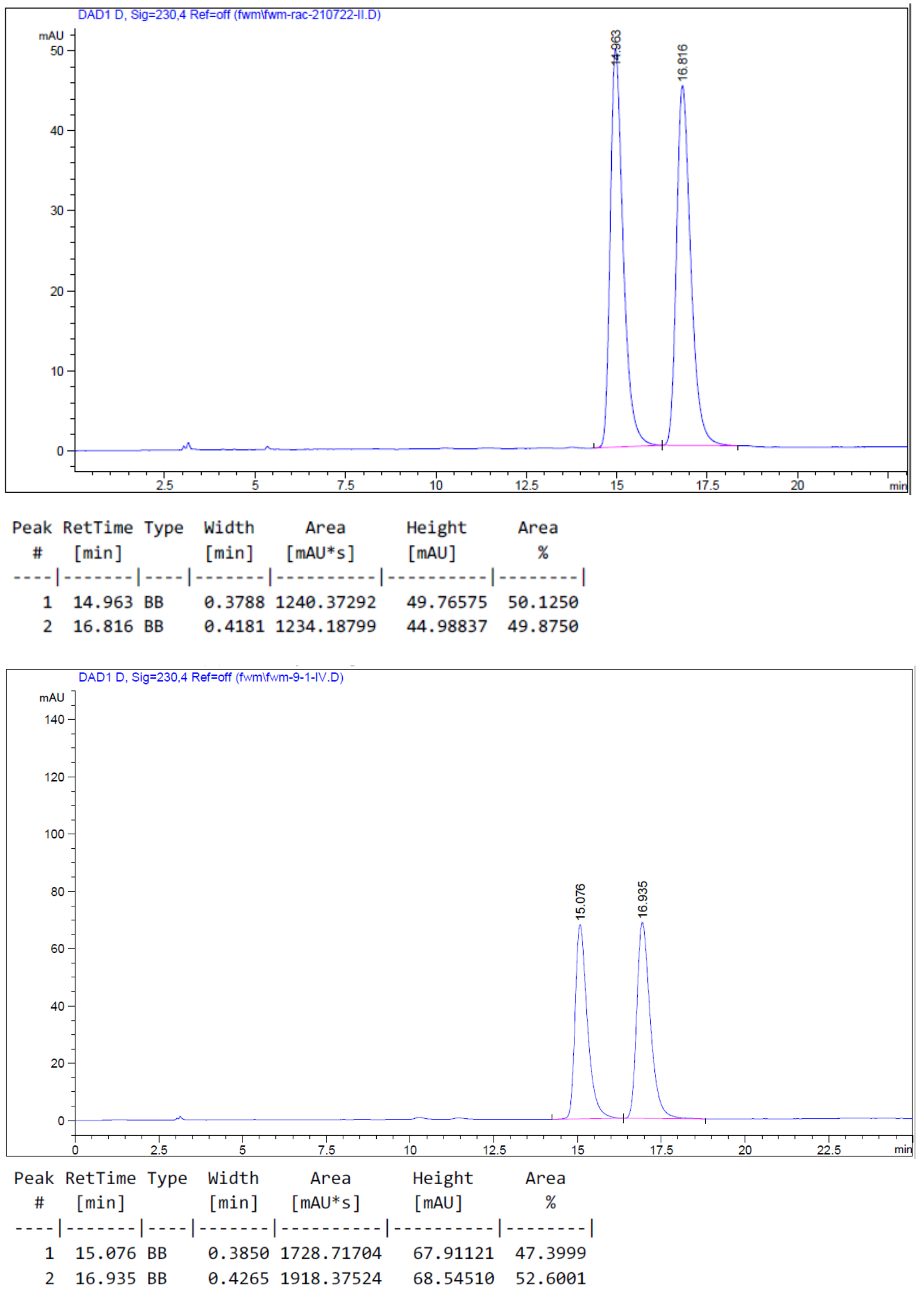


\section{NMR Spectra for New Compounds}

\section{Compound 1b'}
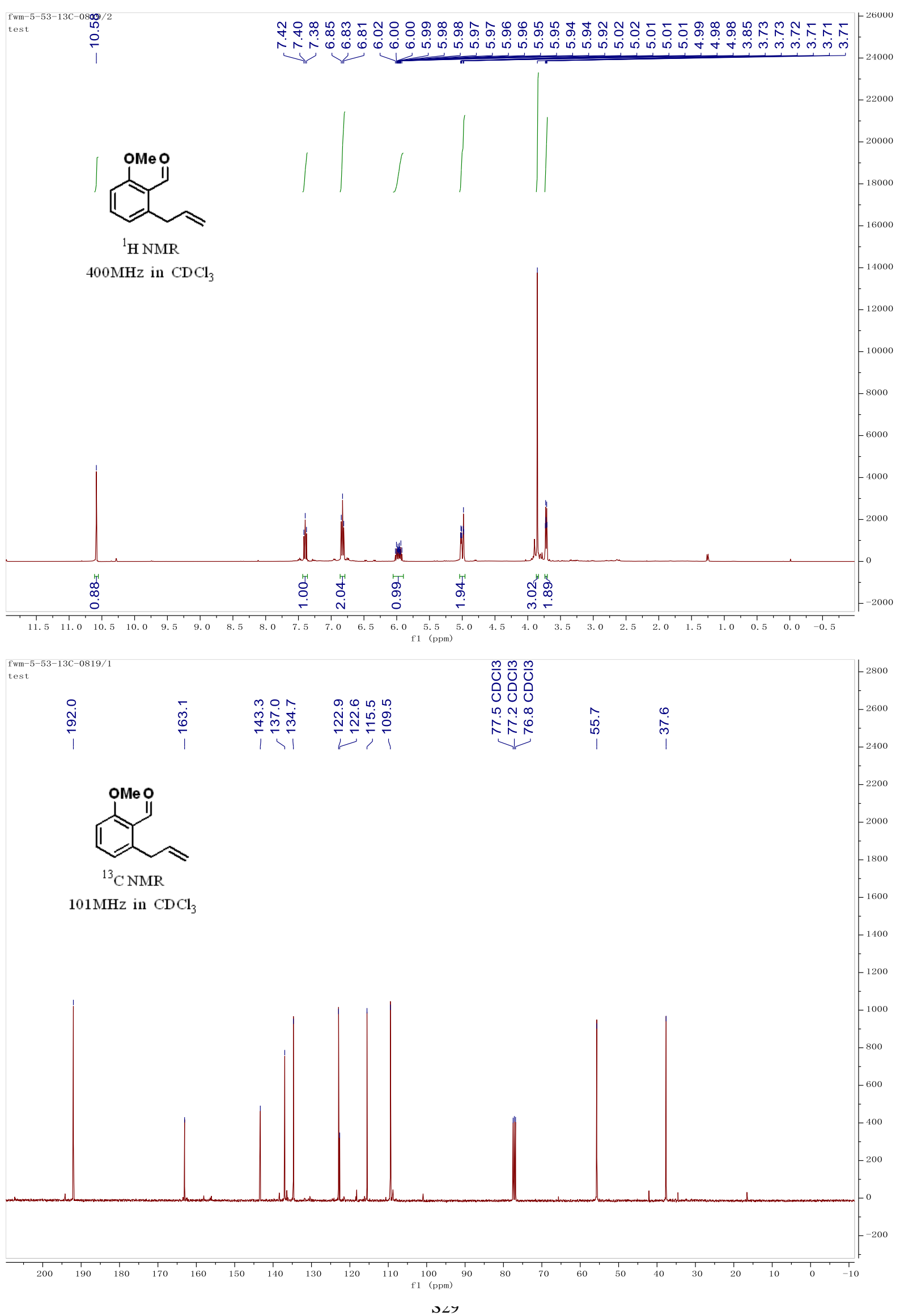


\section{Compound 1c'}
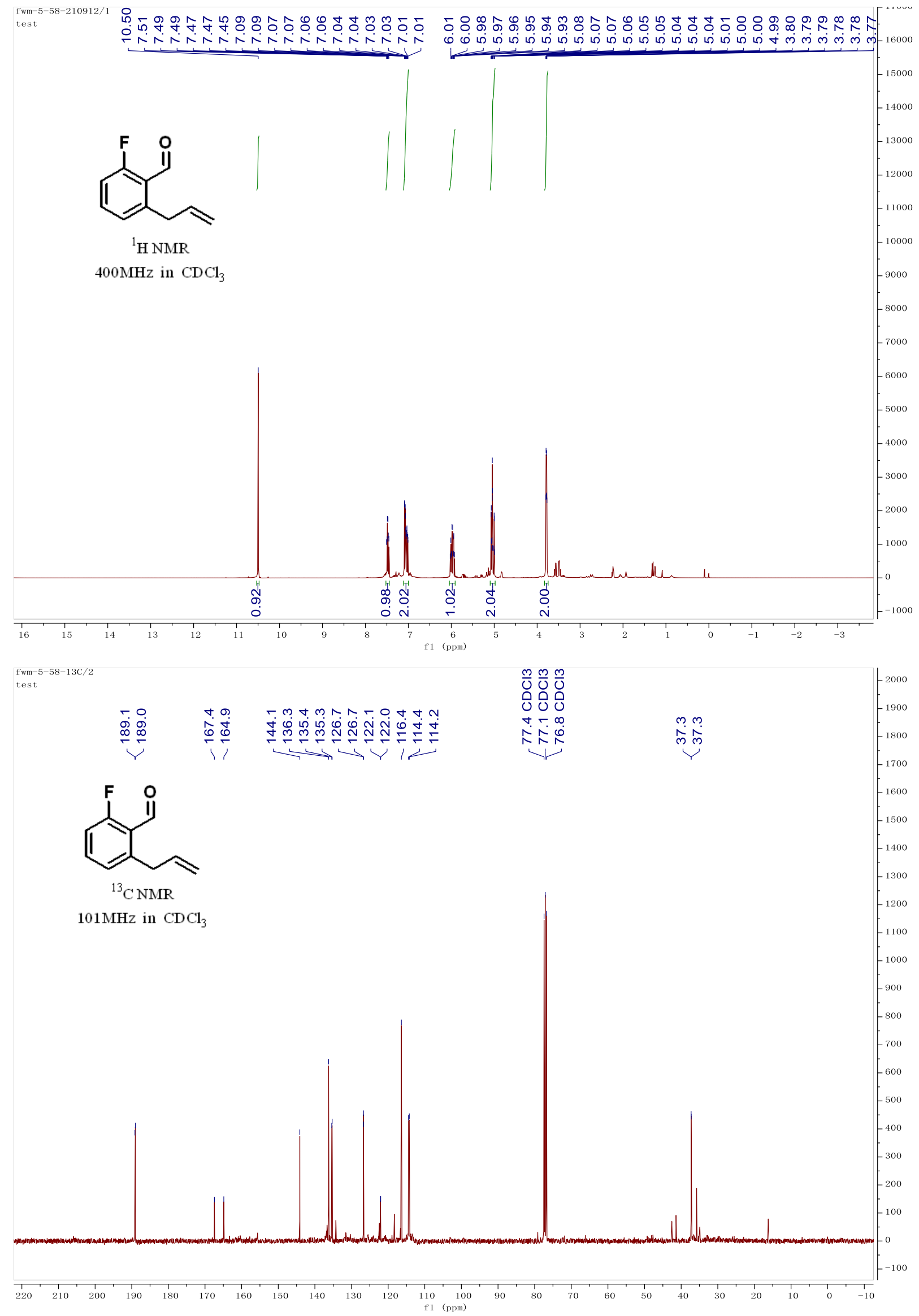


\section{Compound 1g'}
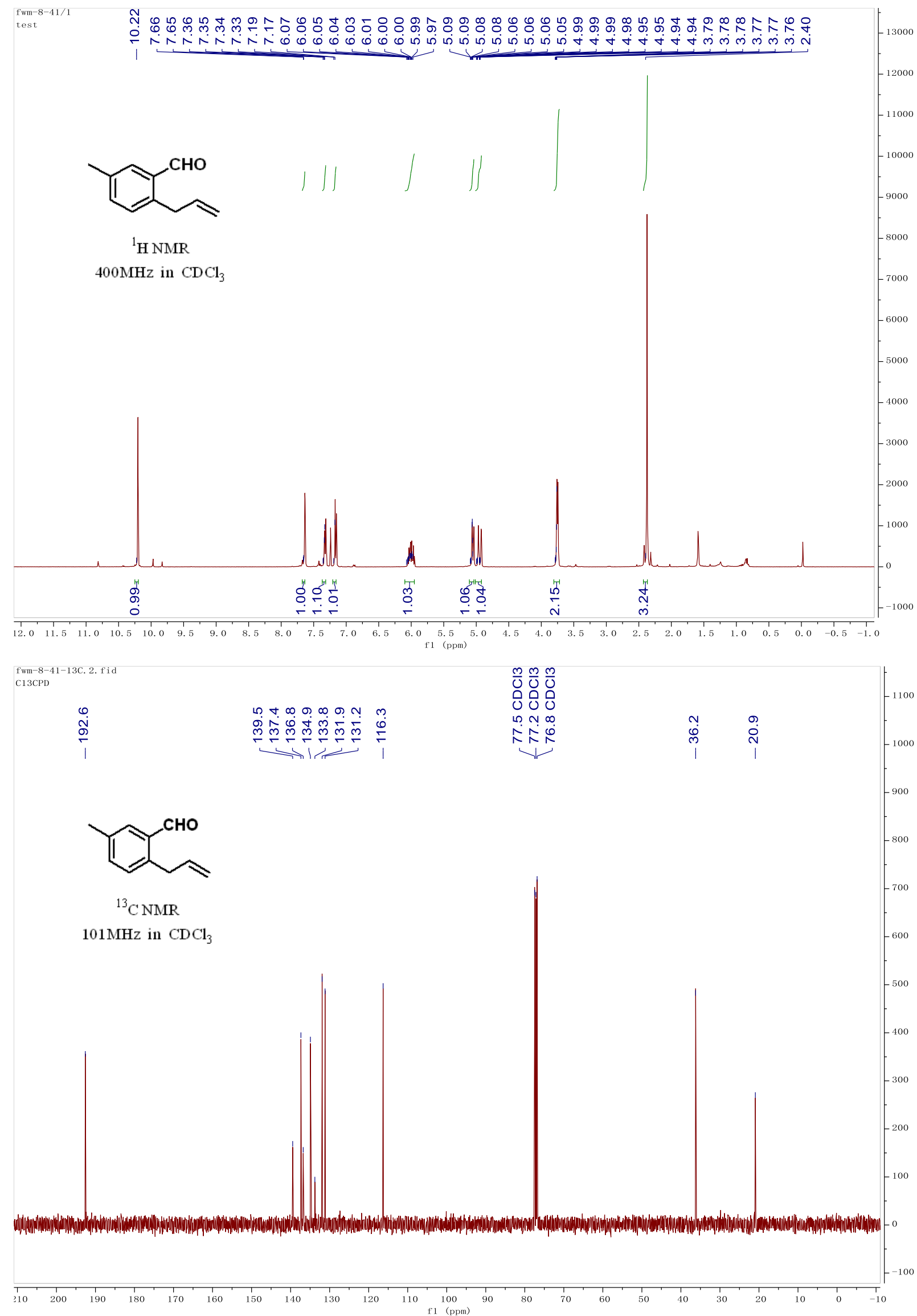


\section{Compound 11'}
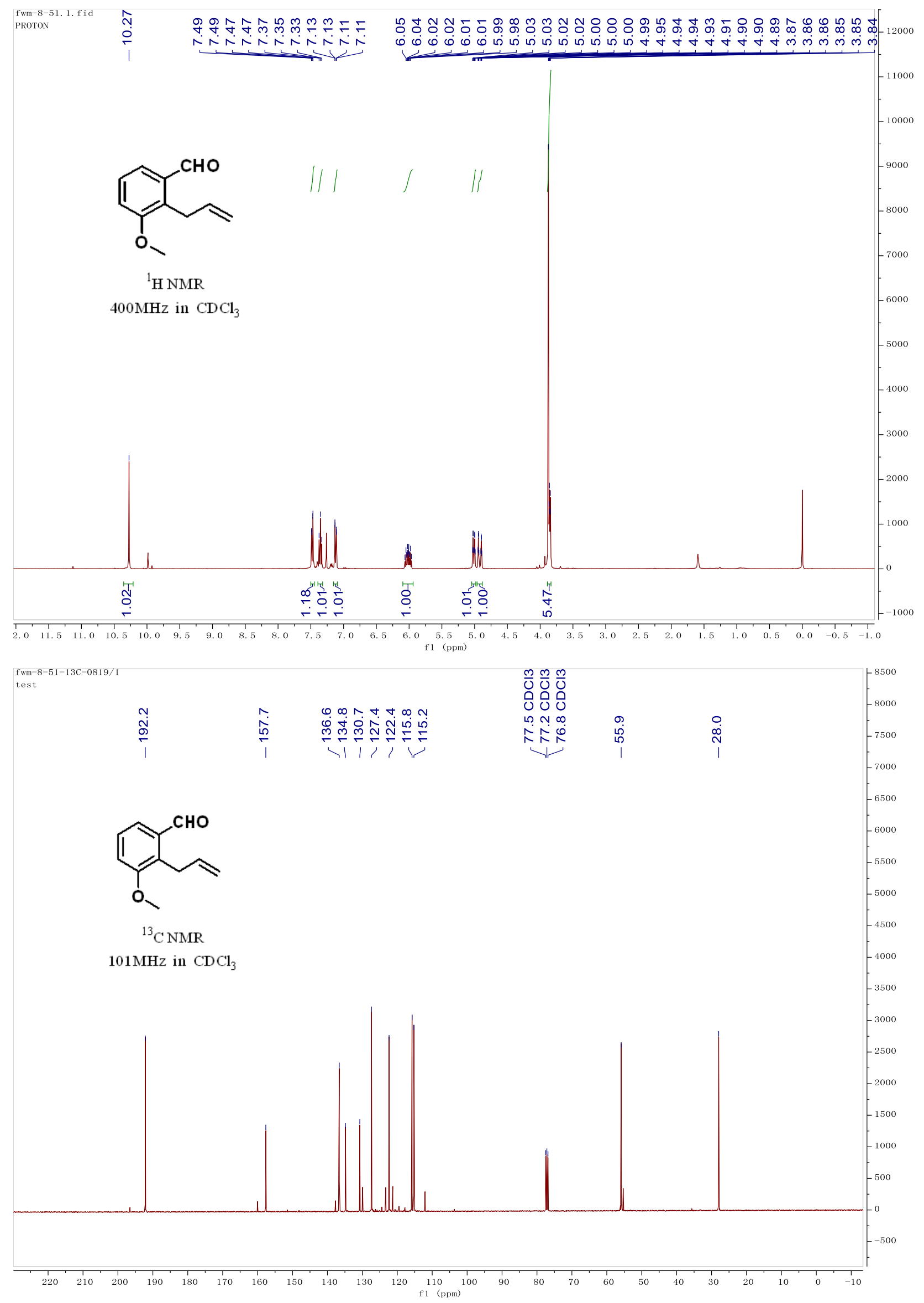


\section{Compound 1m'}
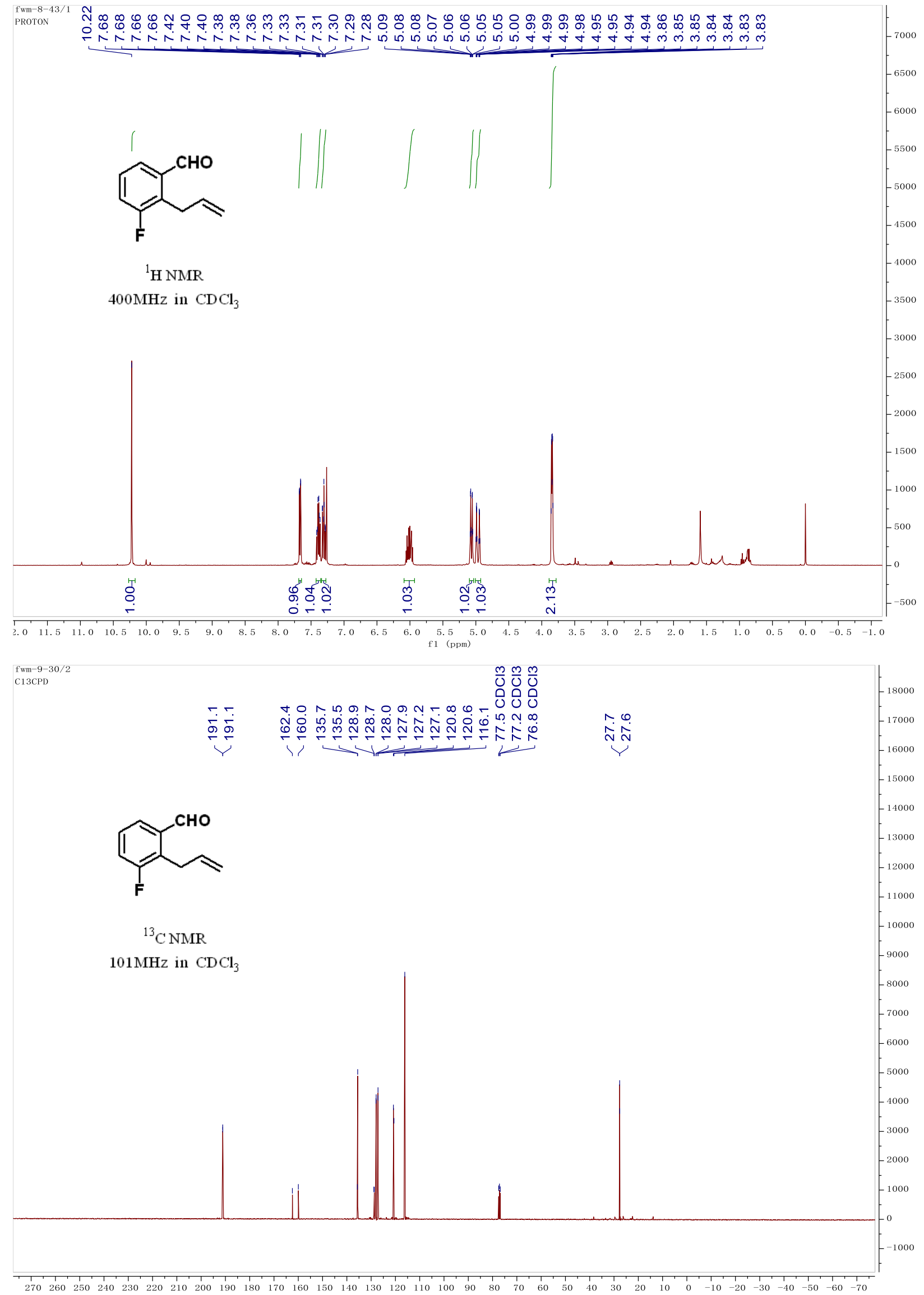

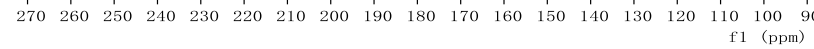




\section{Compound 1n'}
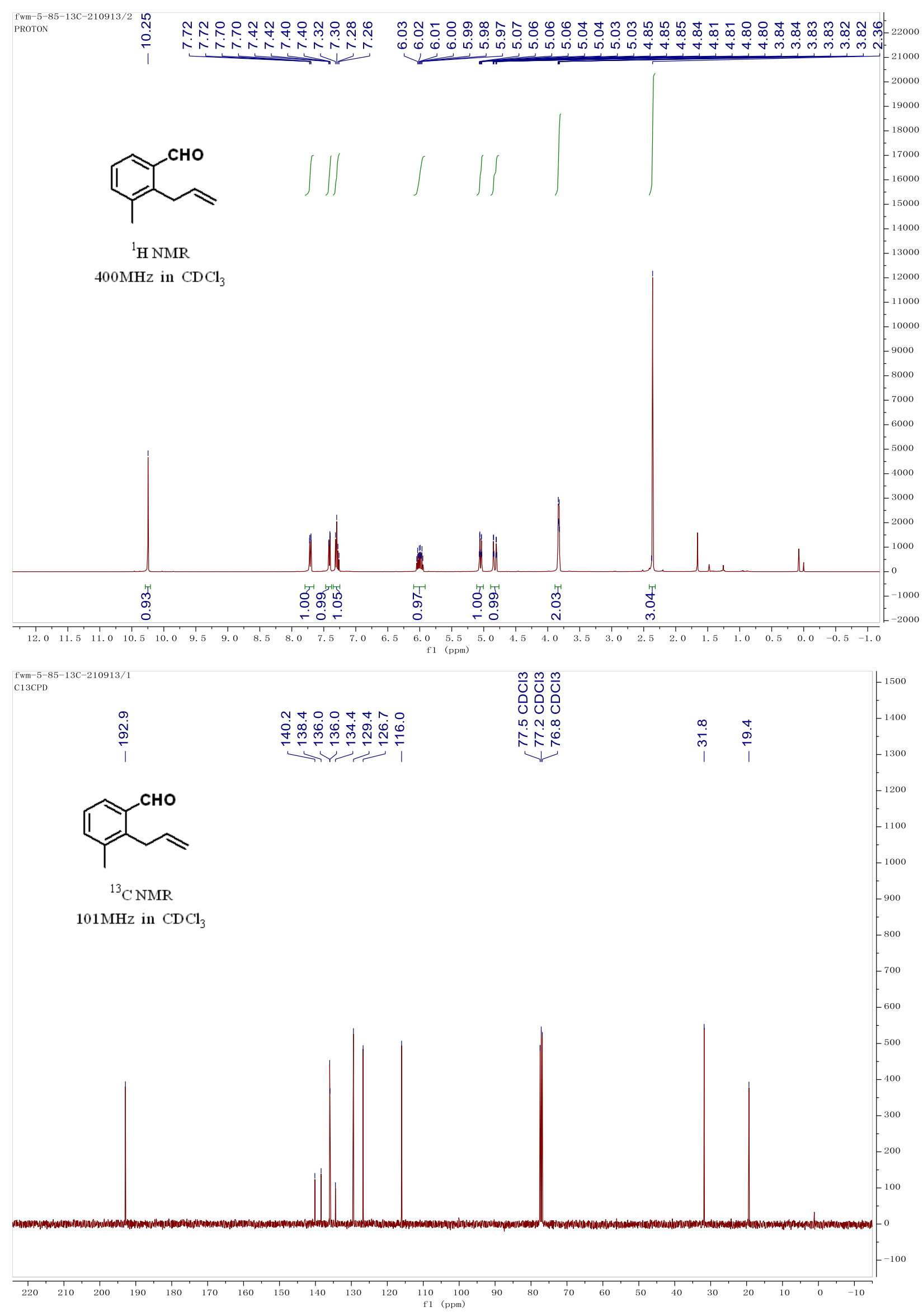


\section{Compound 4d}
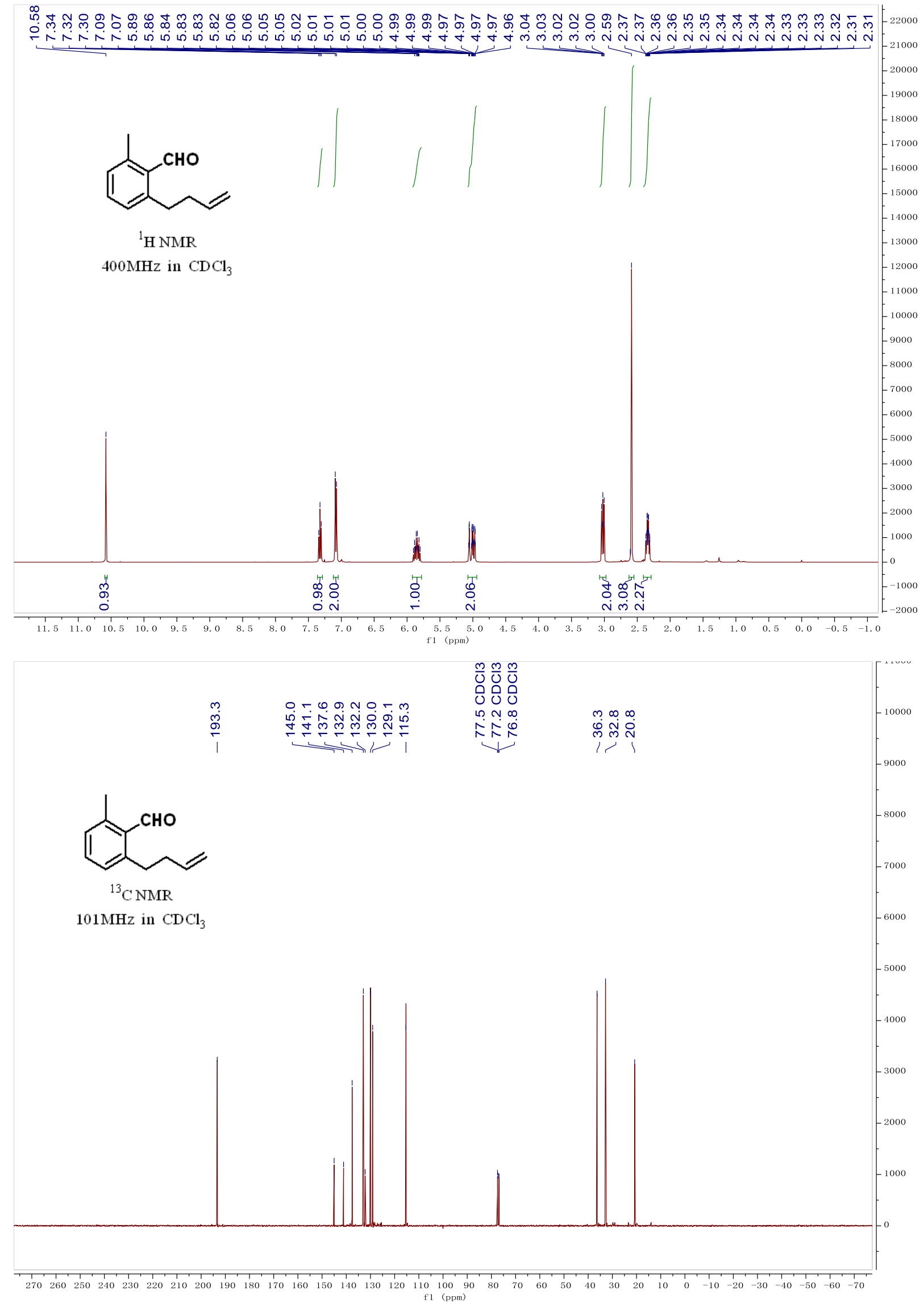


\section{Compound 1b}

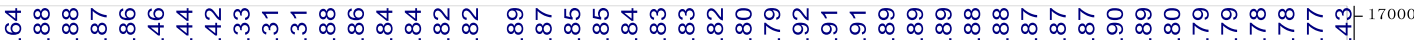

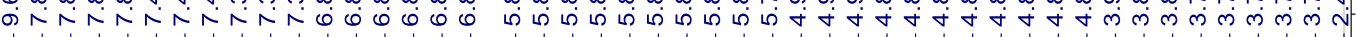
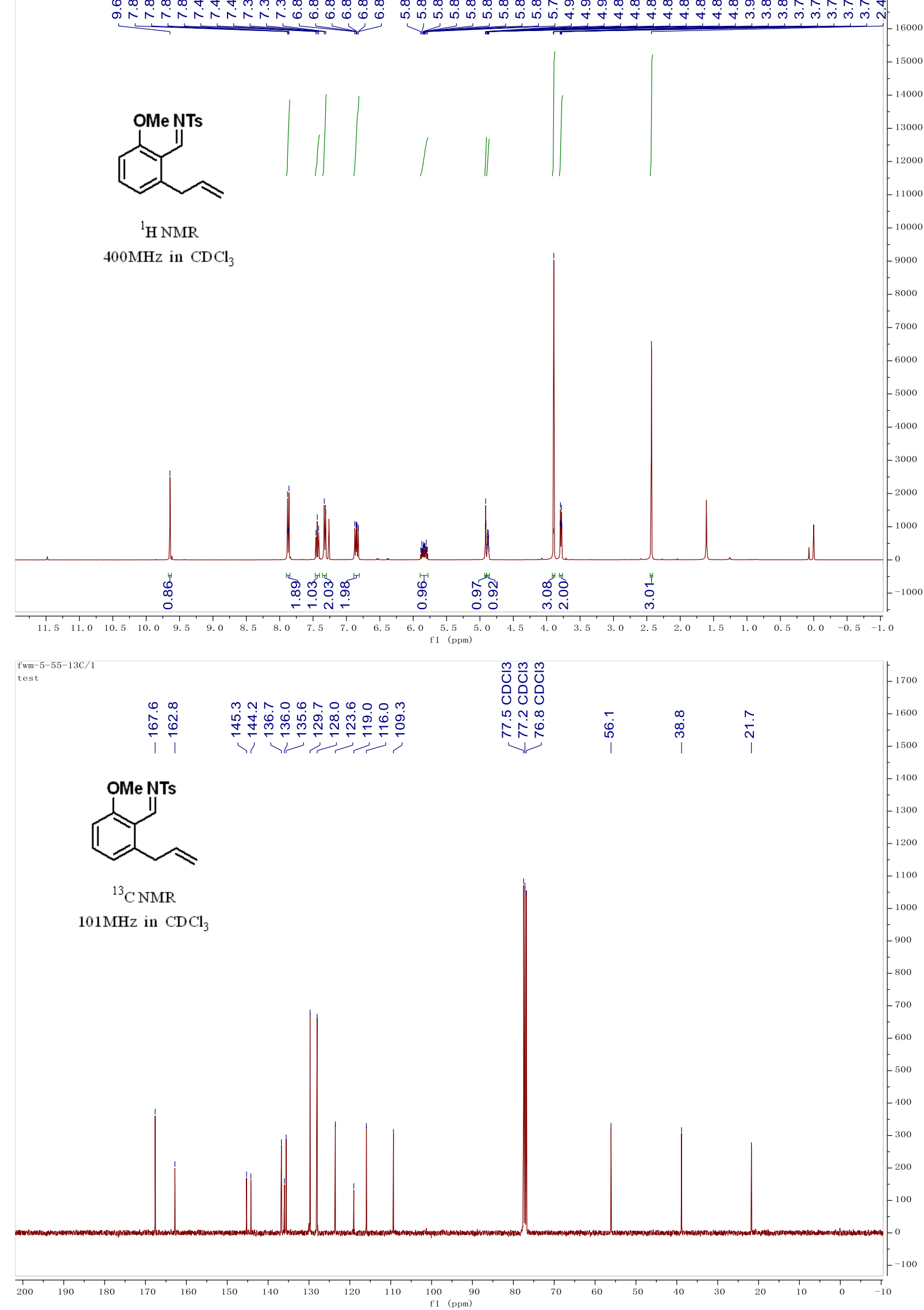


\section{Compound 1c}
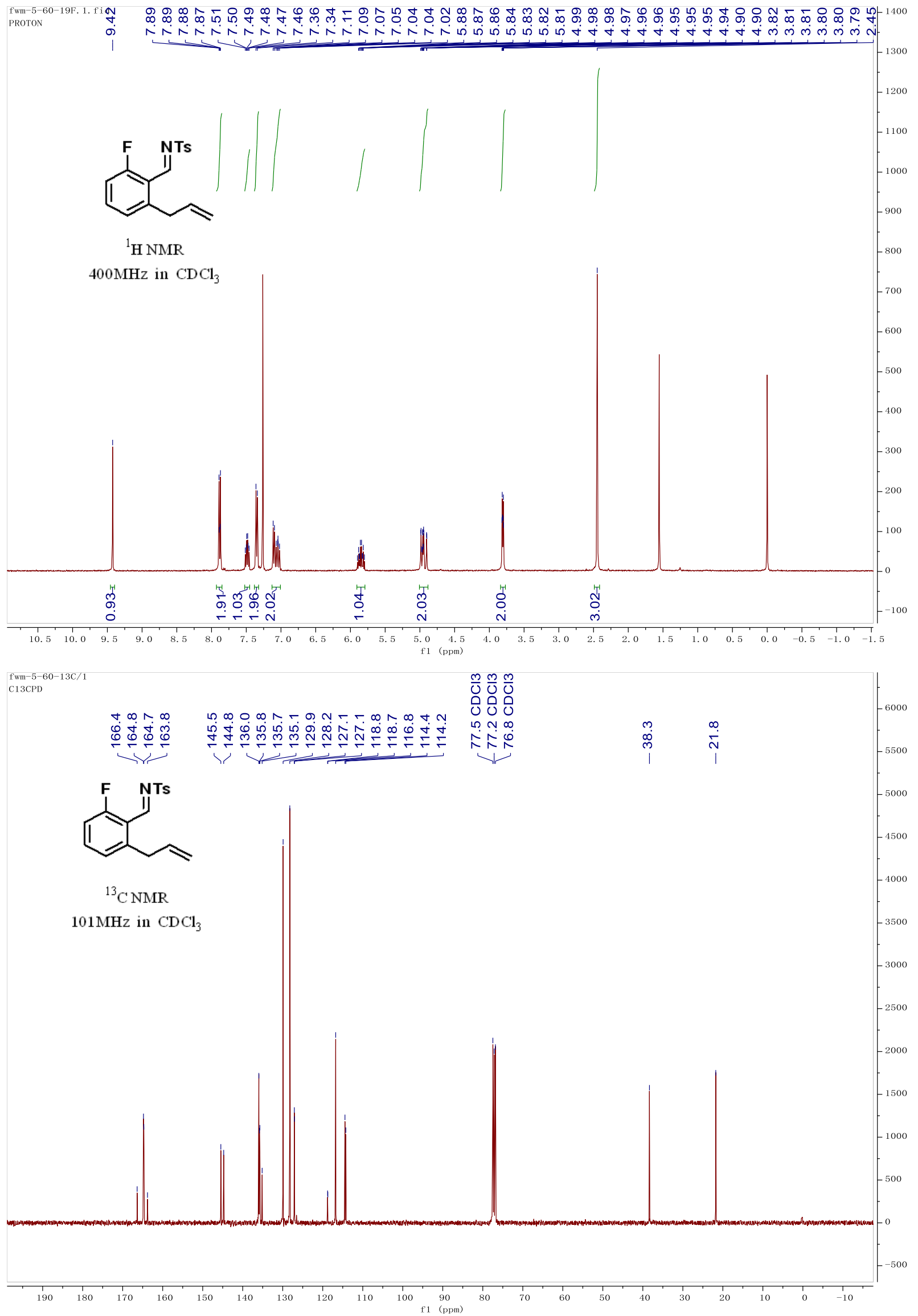


\section{Compound 1d}
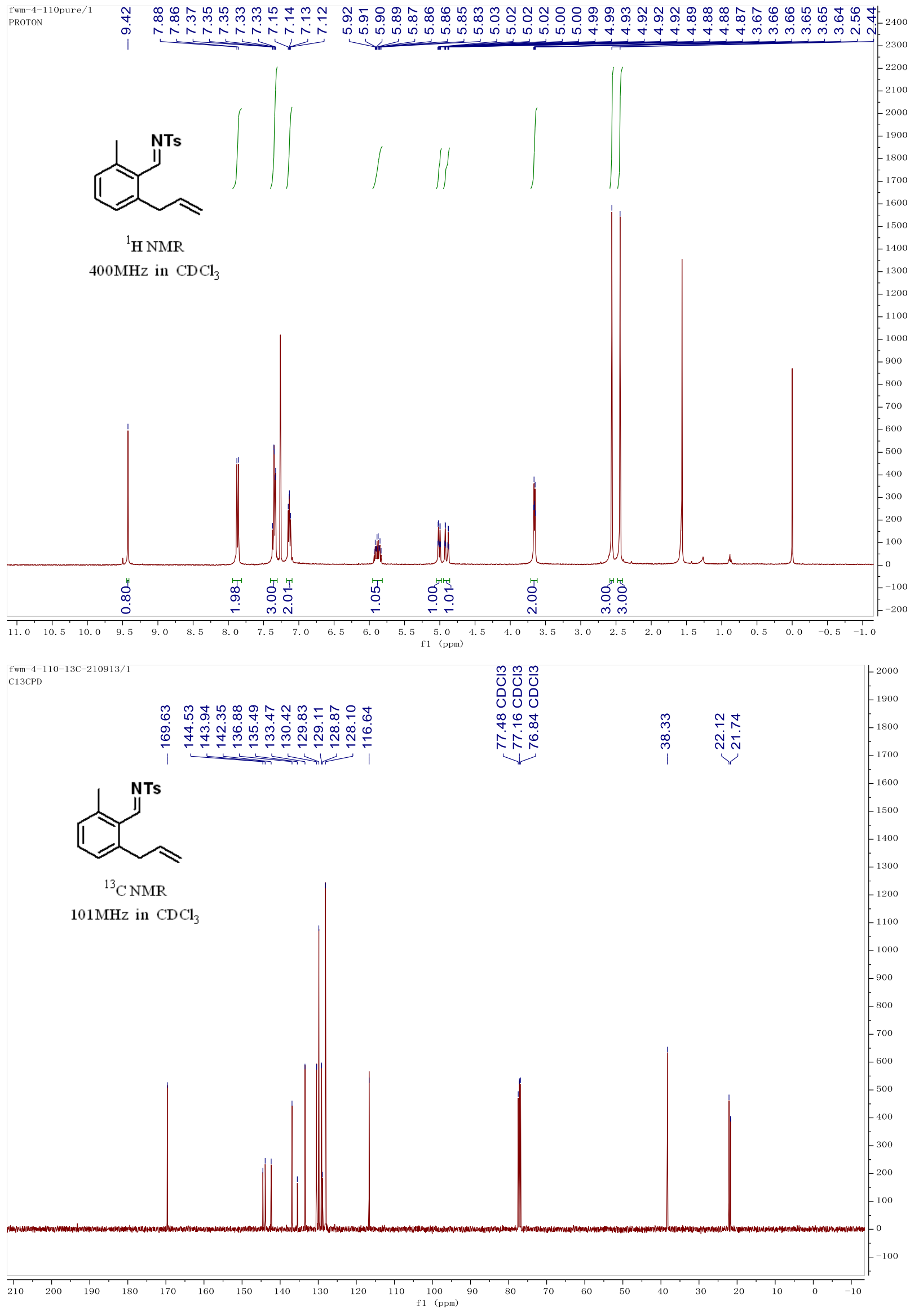


\section{Compound 1f}

仙-

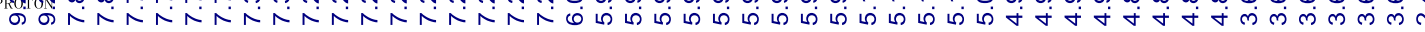
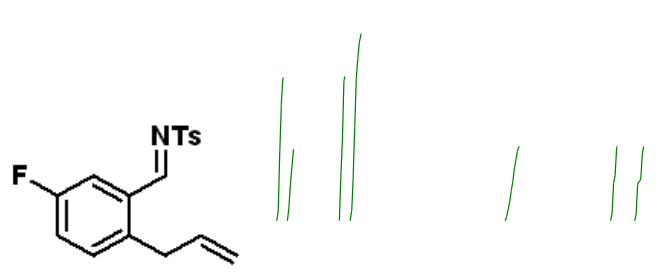

${ }^{1} \mathrm{H}$ NMR

$400 \mathrm{MHz}$ in $\mathrm{CDCl}_{3}$
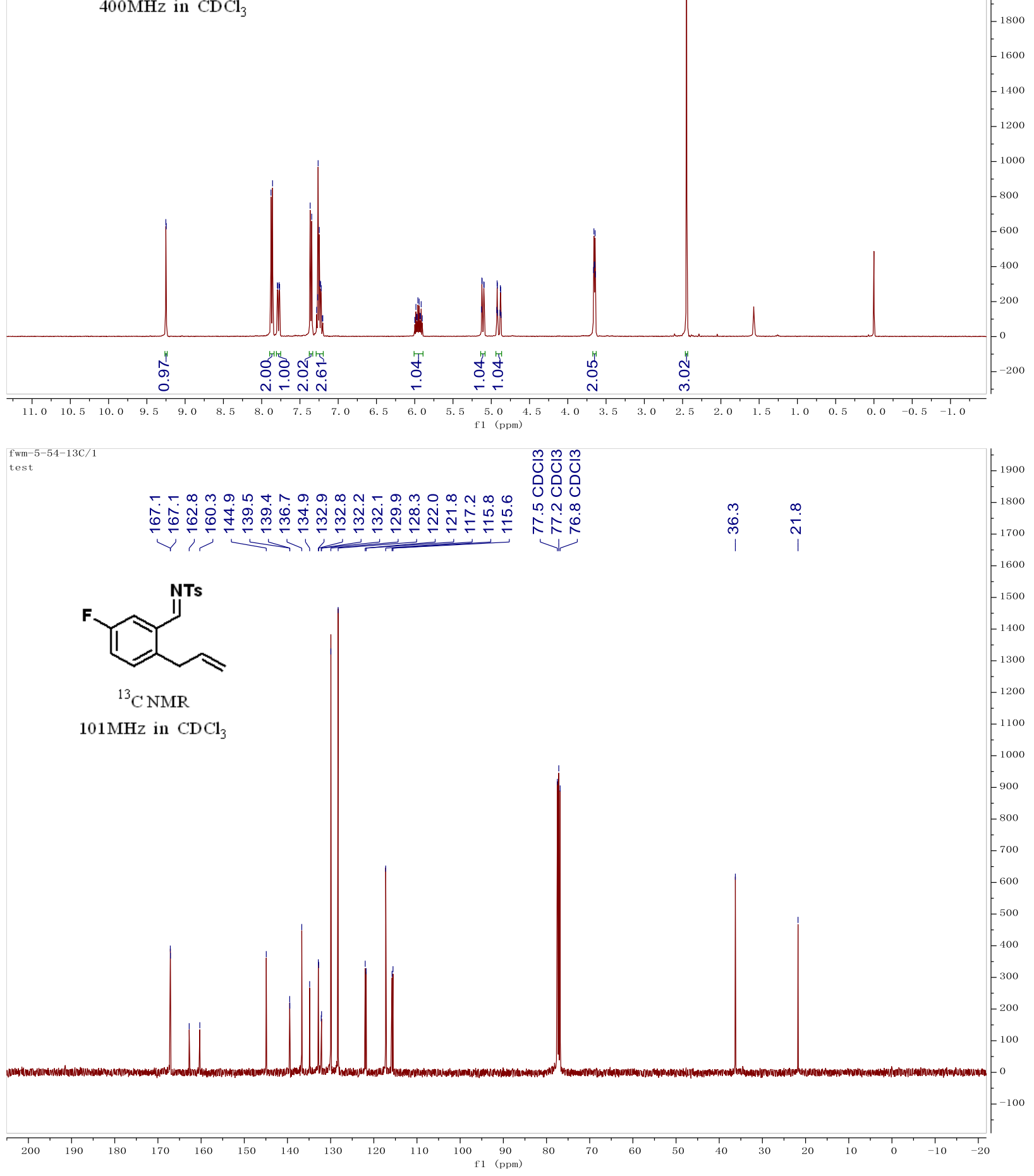


\section{Compound 1g}
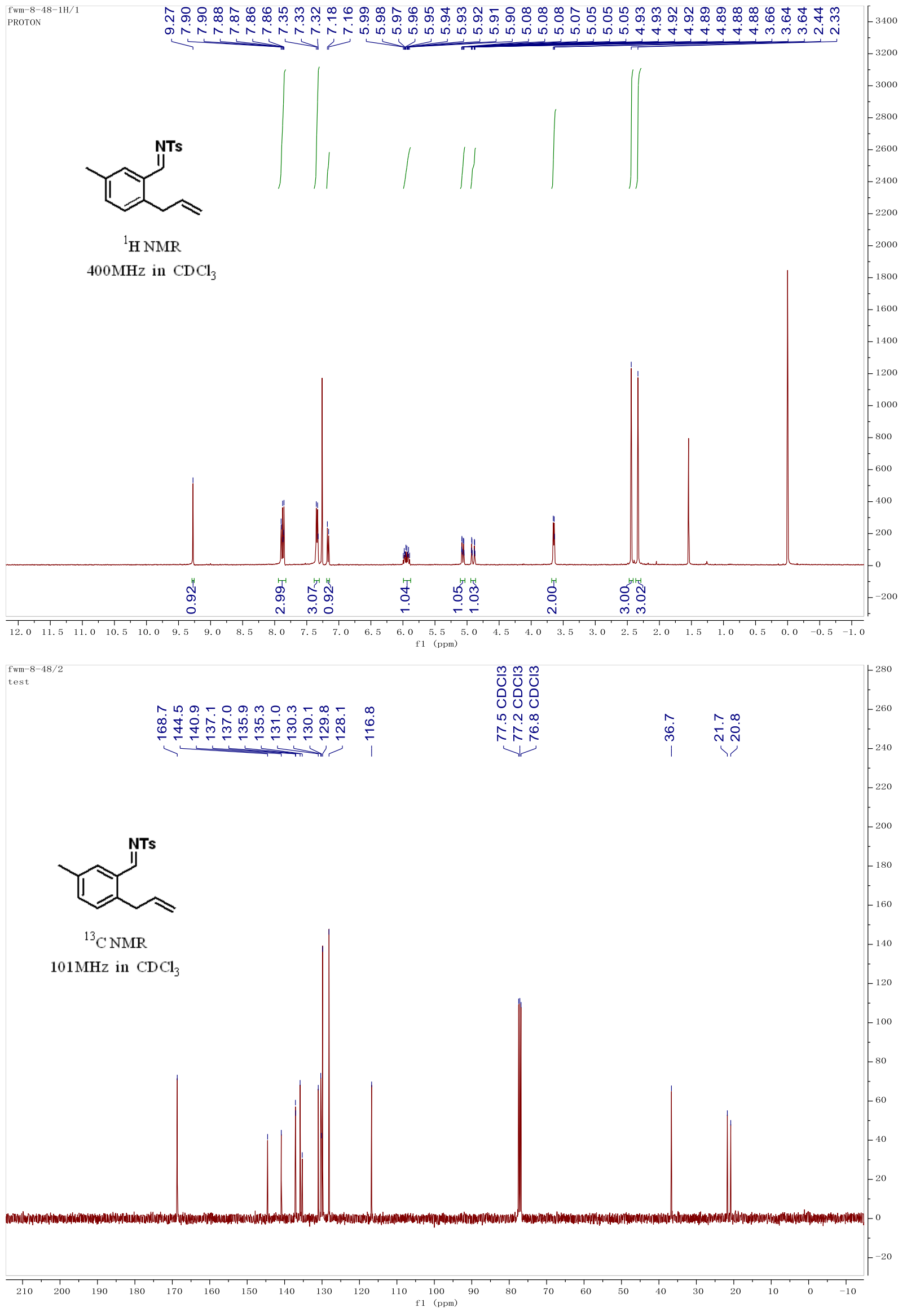


\section{Compound $1 \mathrm{~h}$}

Pwm-7-162-13C/N

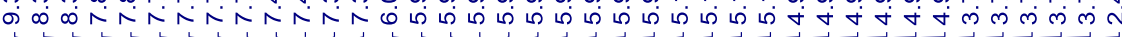
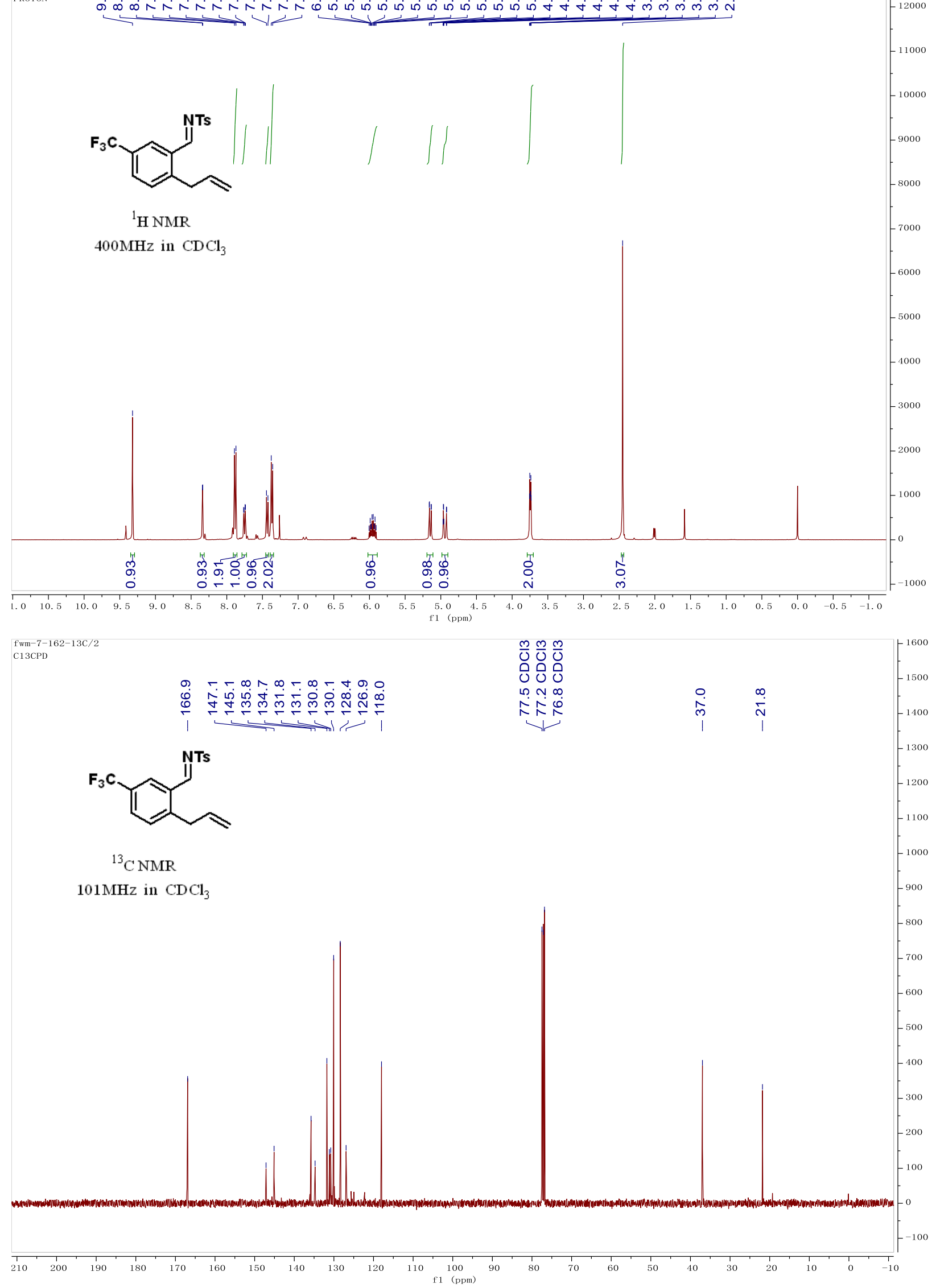


\section{Compound 1i}

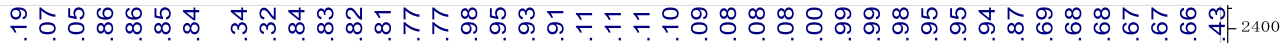
ヘ
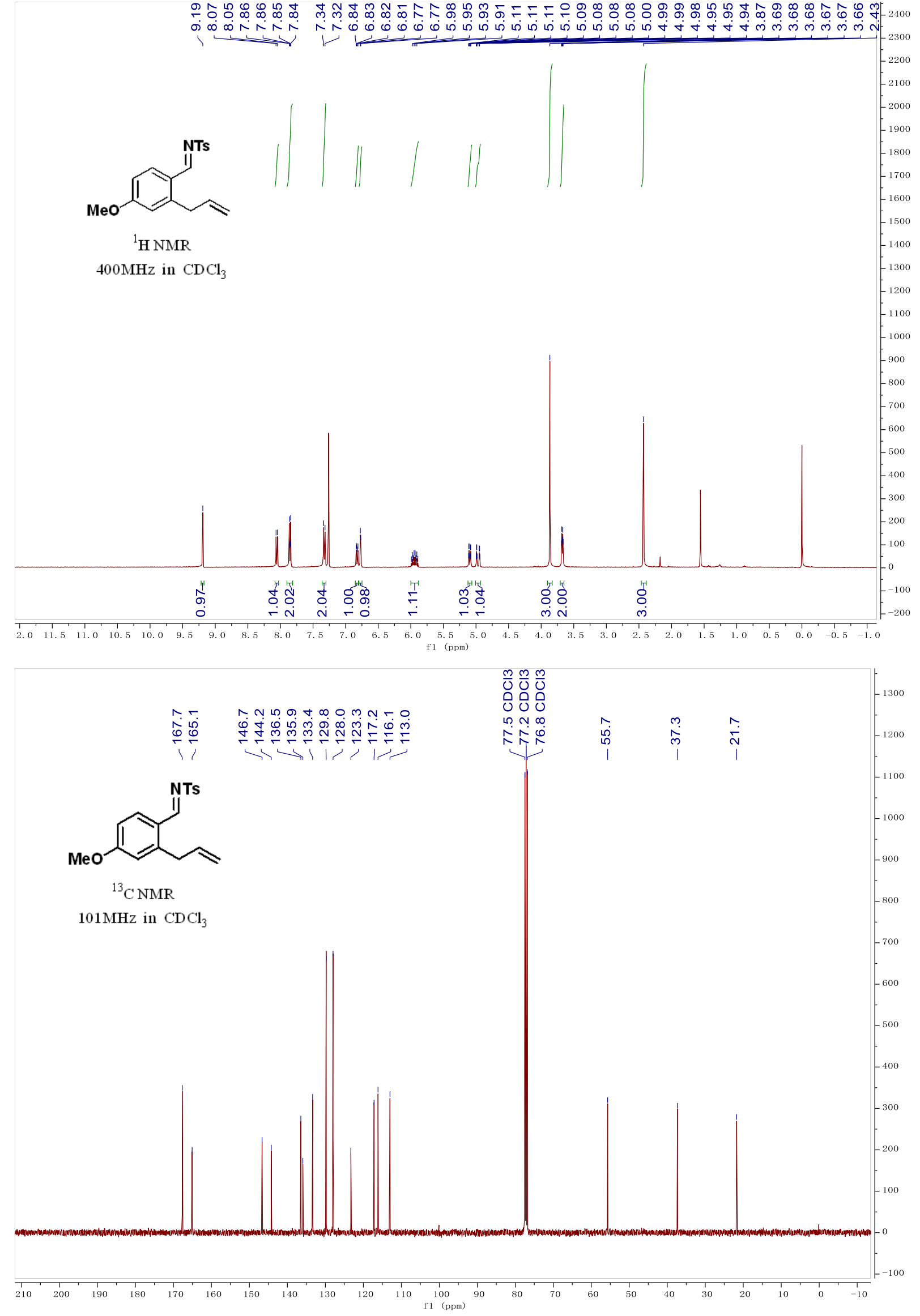


\section{Compound 1k}

مุ

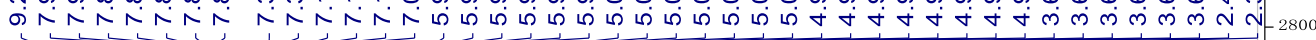<smiles>C=CCc1cc(C)ccc1C=[N+]=[N-]</smiles>

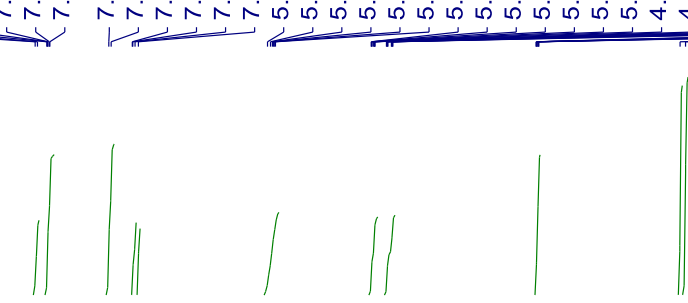

${ }^{1} \mathrm{H} \mathrm{NMR}$

$400 \mathrm{MHz}$ in $\mathrm{CDCl}_{3}$
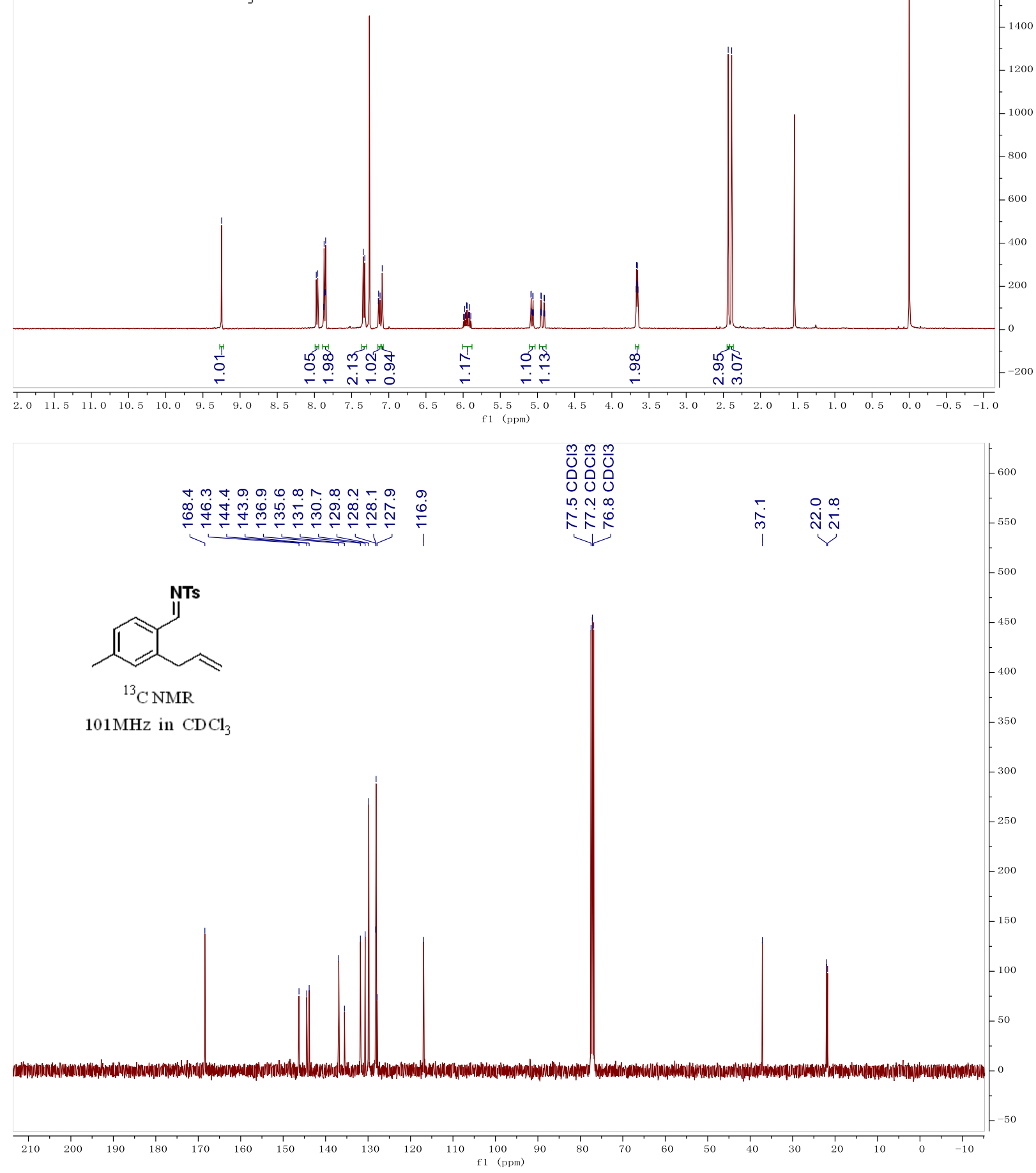


\section{Compound 11}
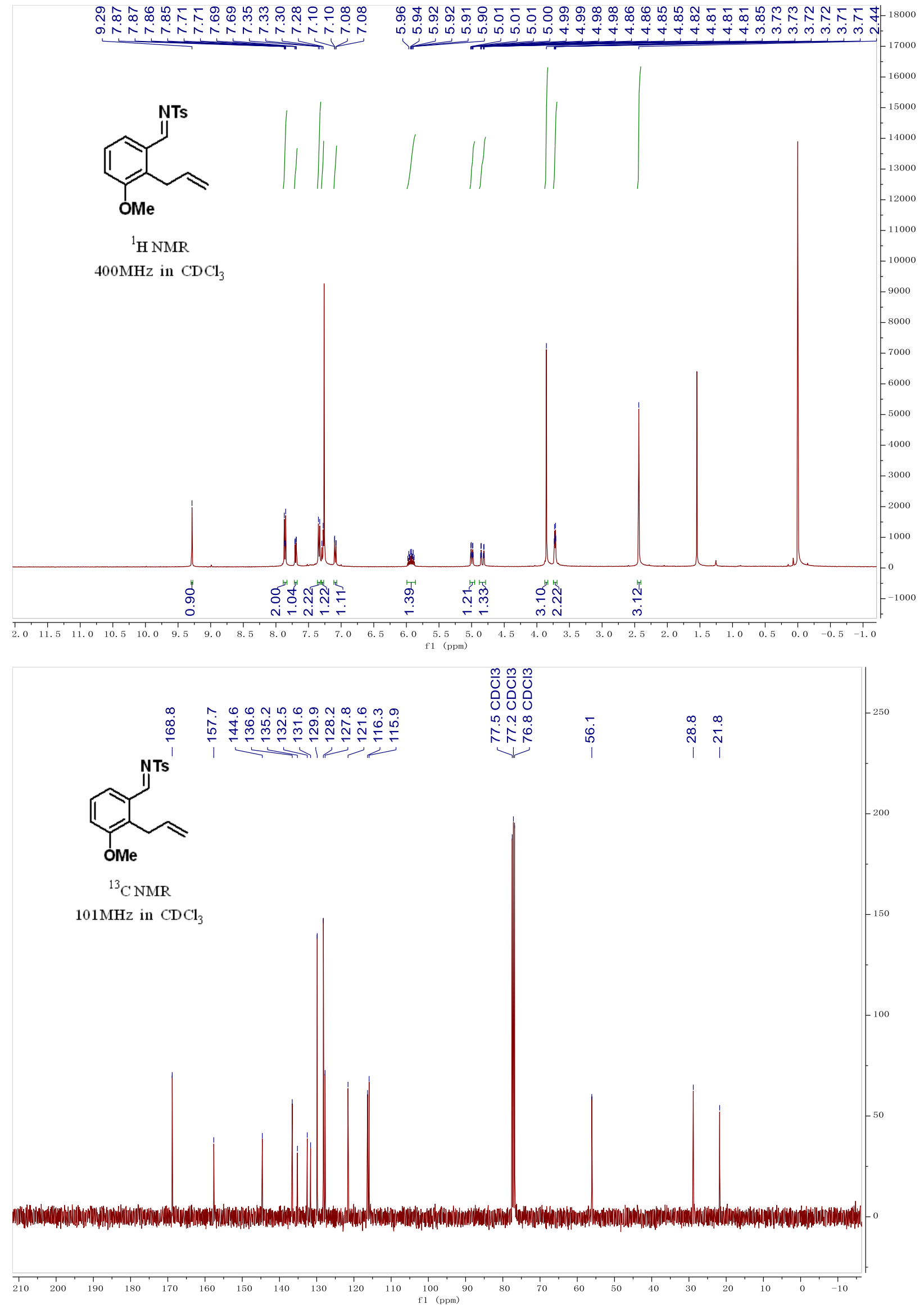


\section{Compound 1m}
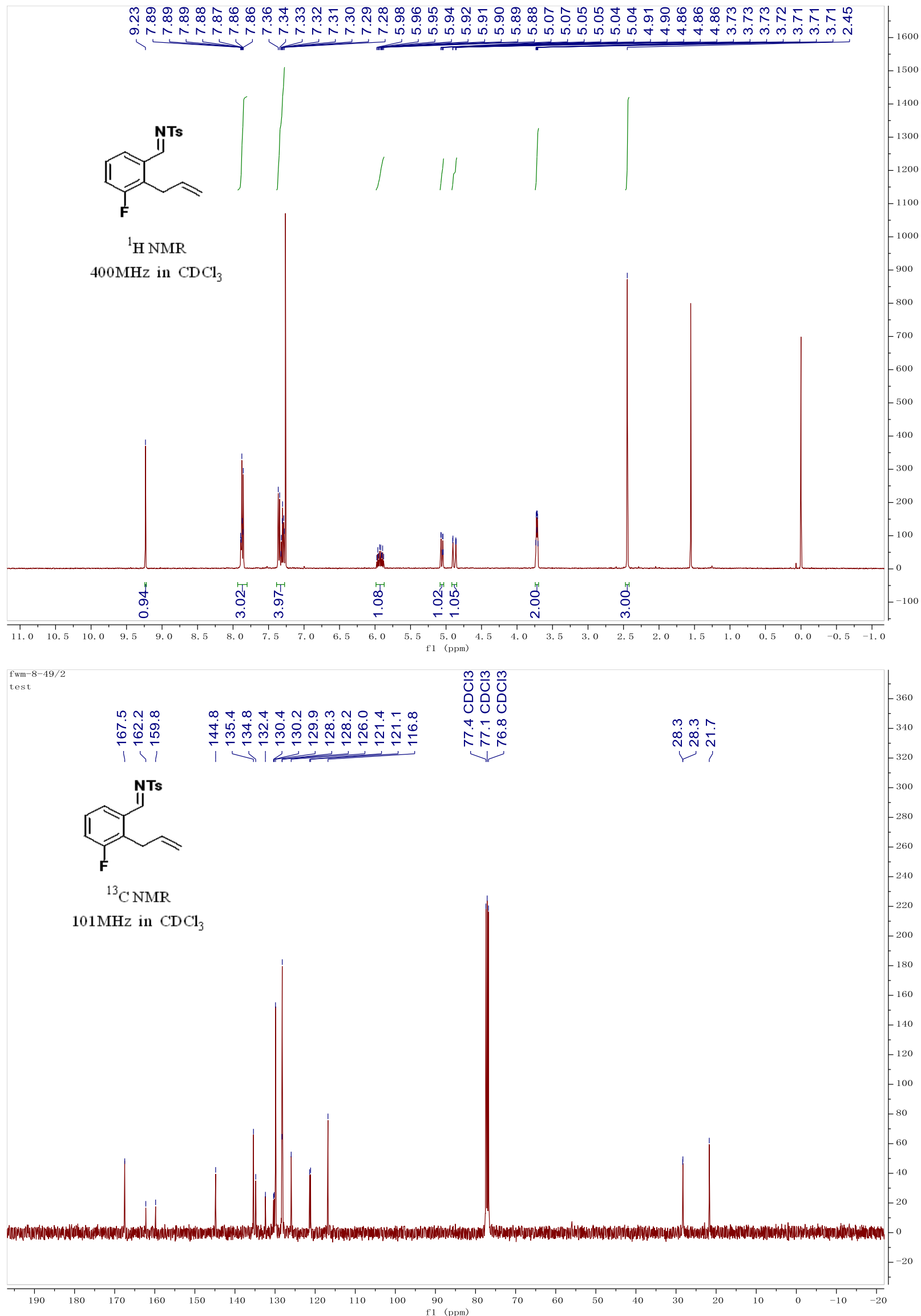


\section{Compound 1n}
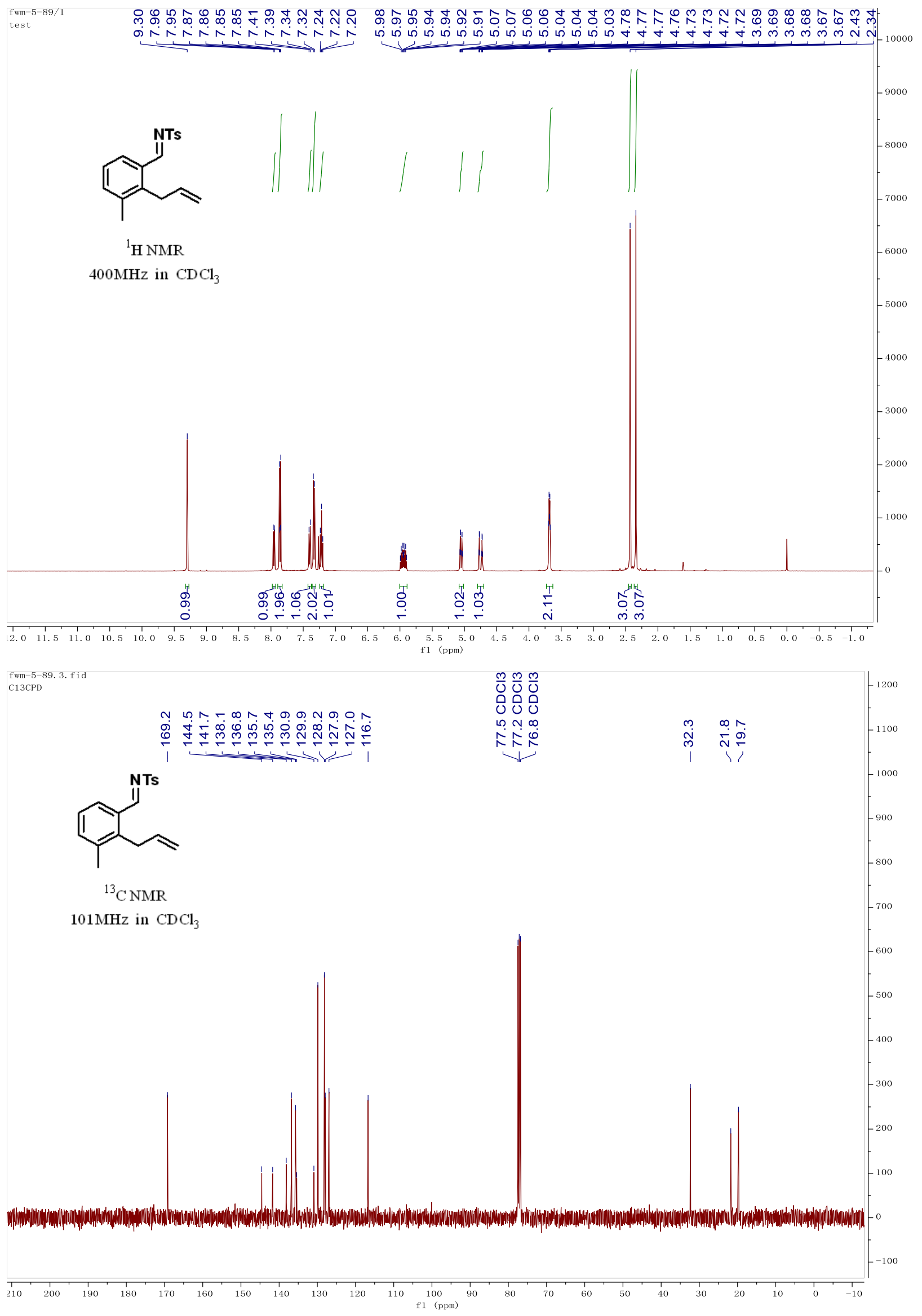


\section{Compound 1s}

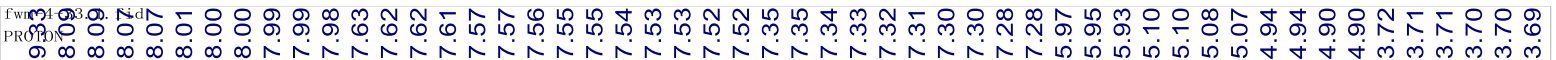

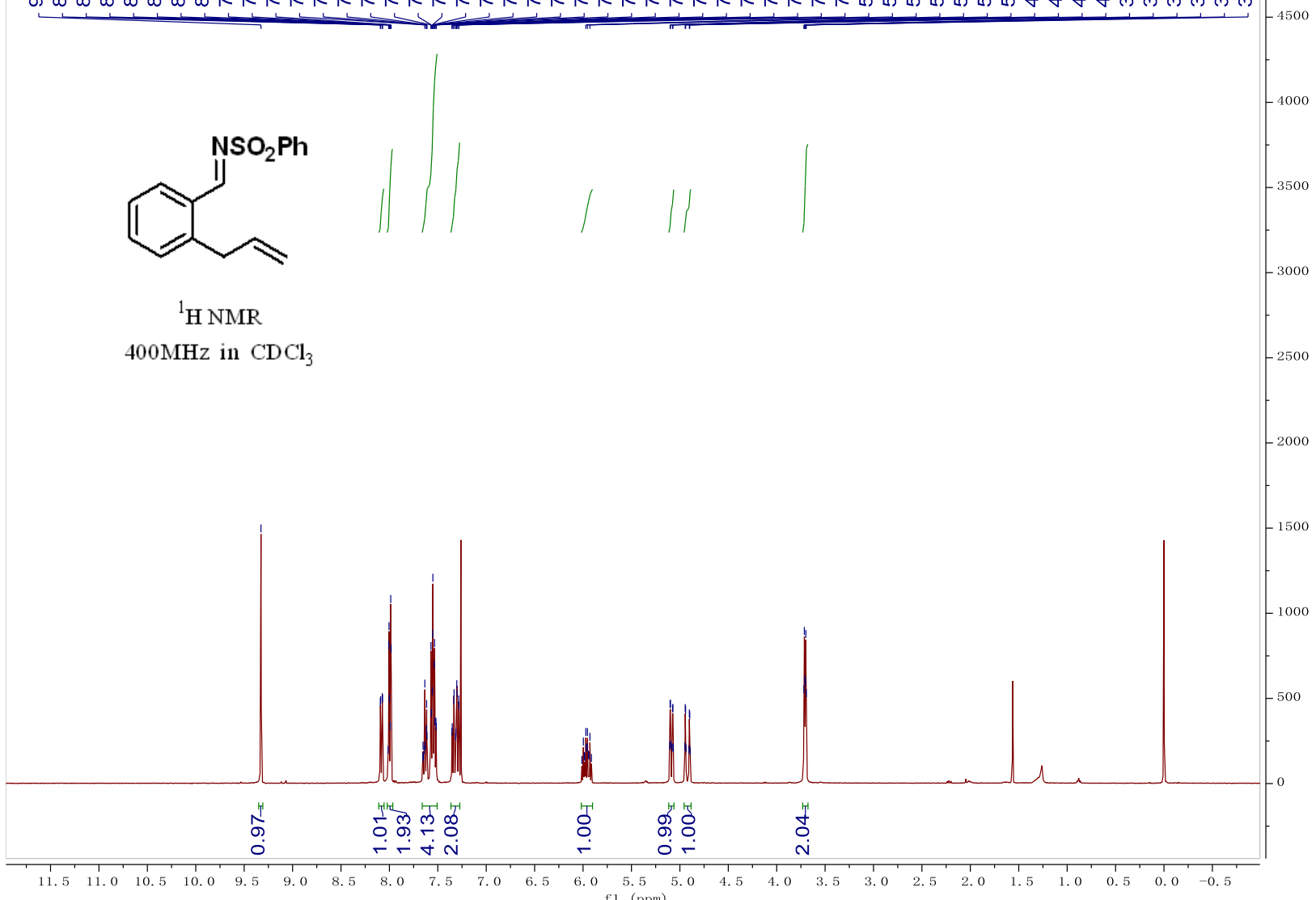

fwm-4-33-13C/1

○)

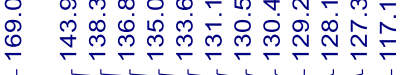

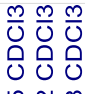

in

圭定

袥

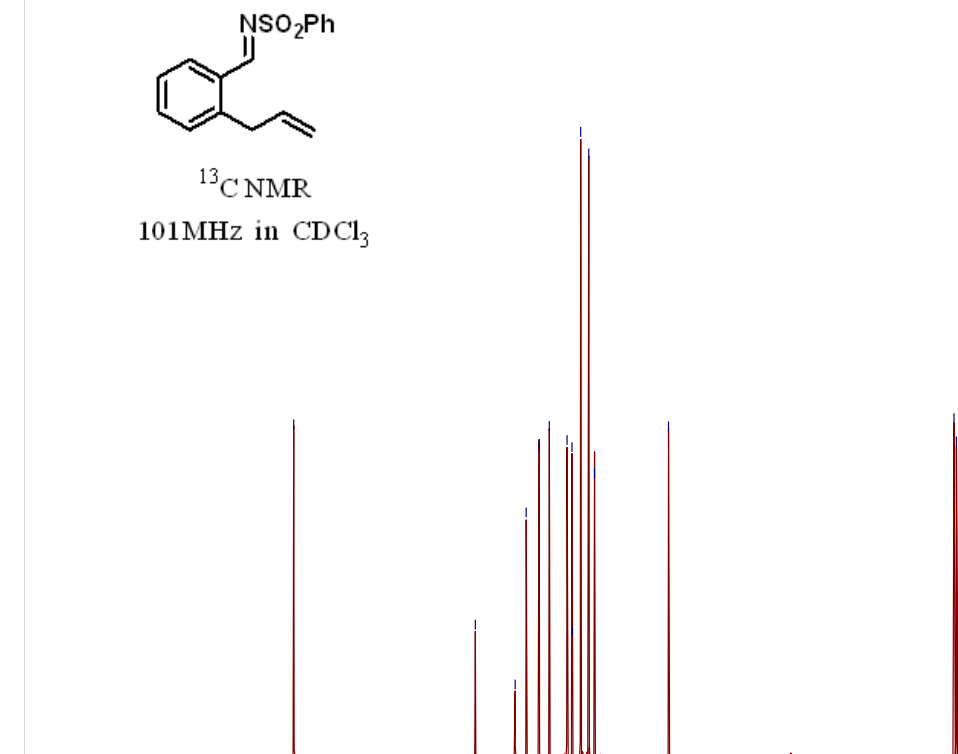

${ }^{13} \mathrm{CNMR}$

$101 \mathrm{MHz}$ in $\mathrm{CDCl}_{3}$
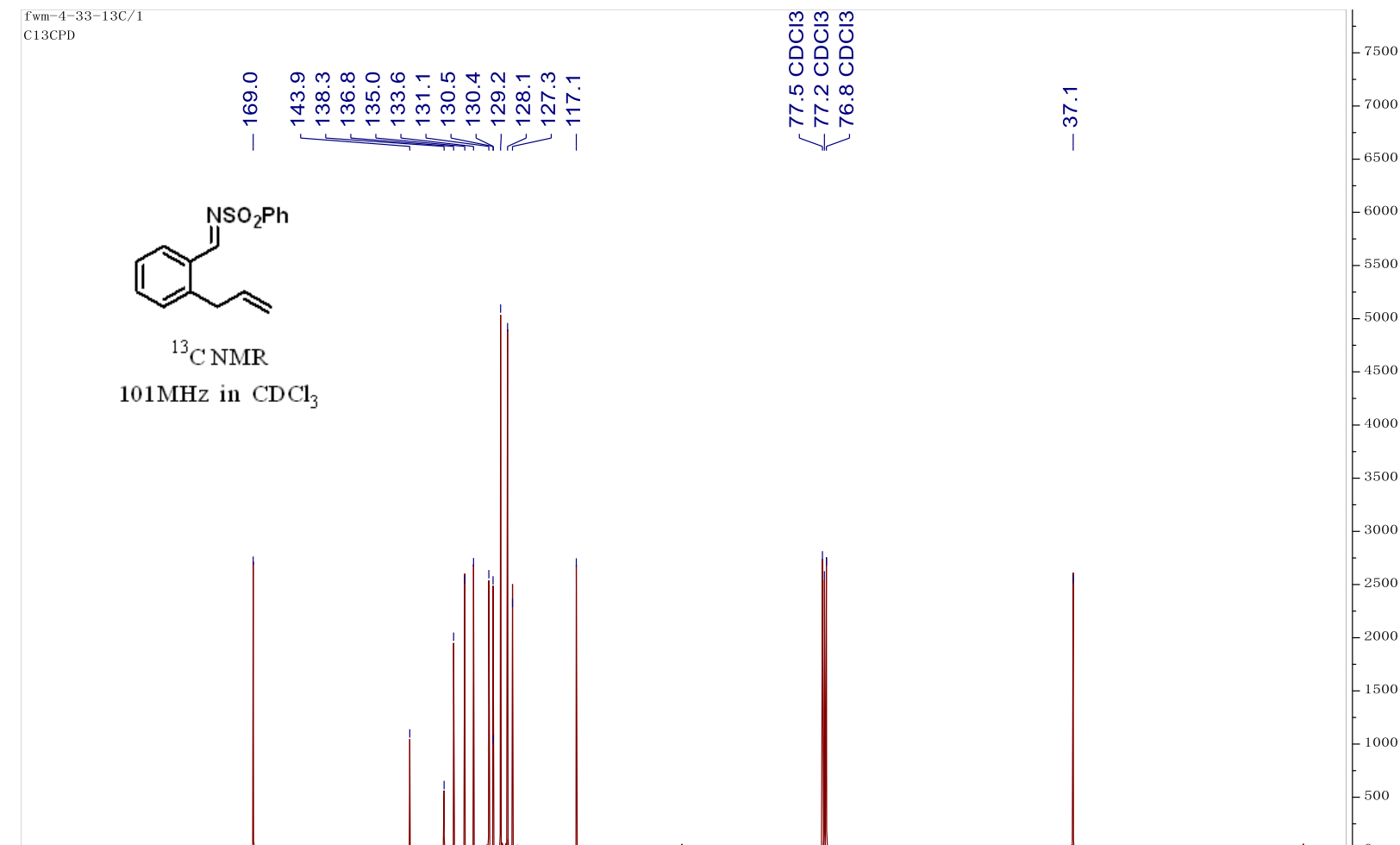


\section{Compound 1r}

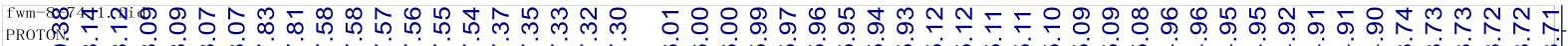

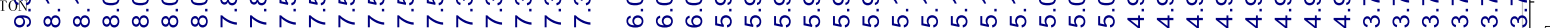
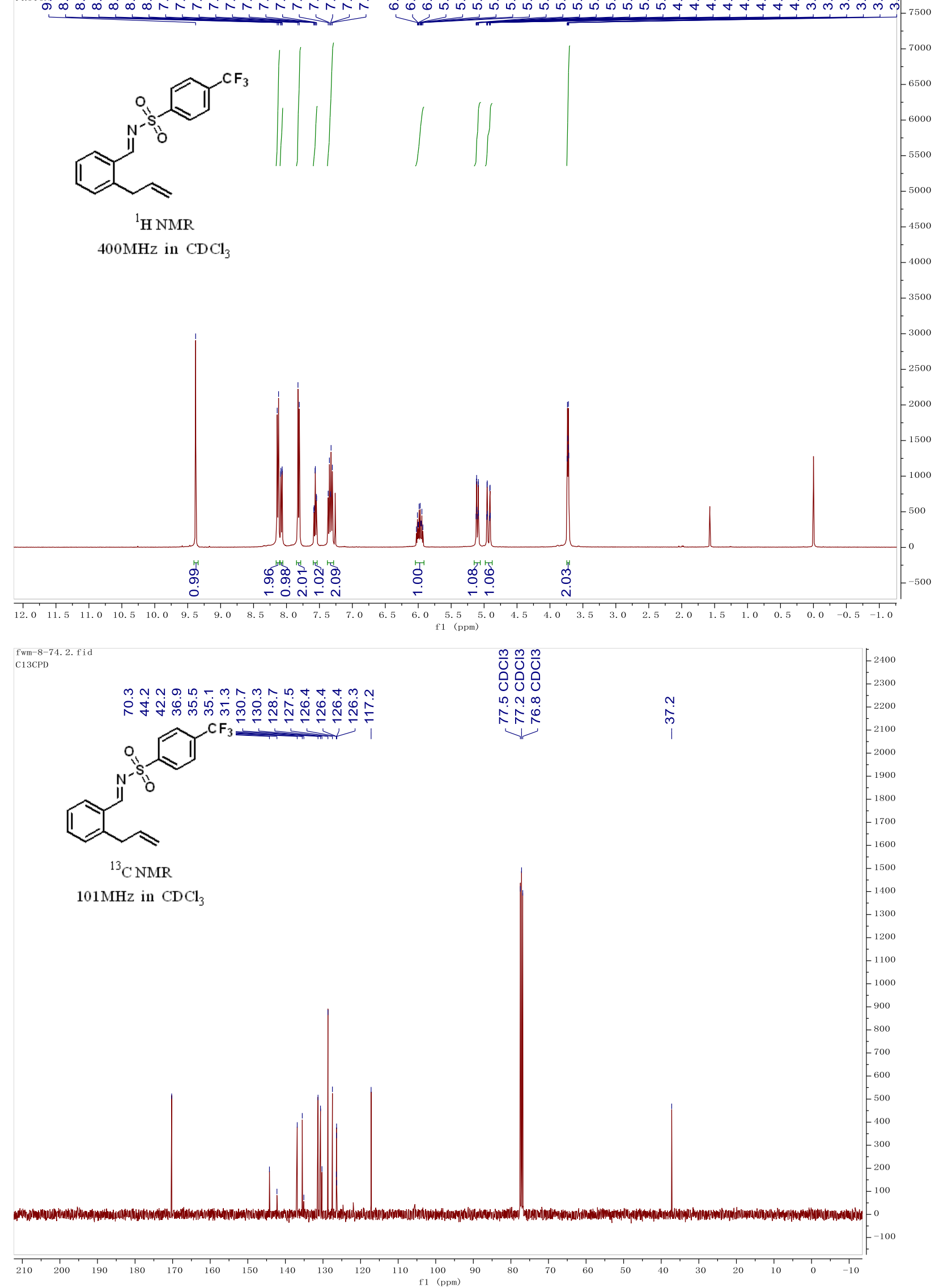


\section{Compound 1t}
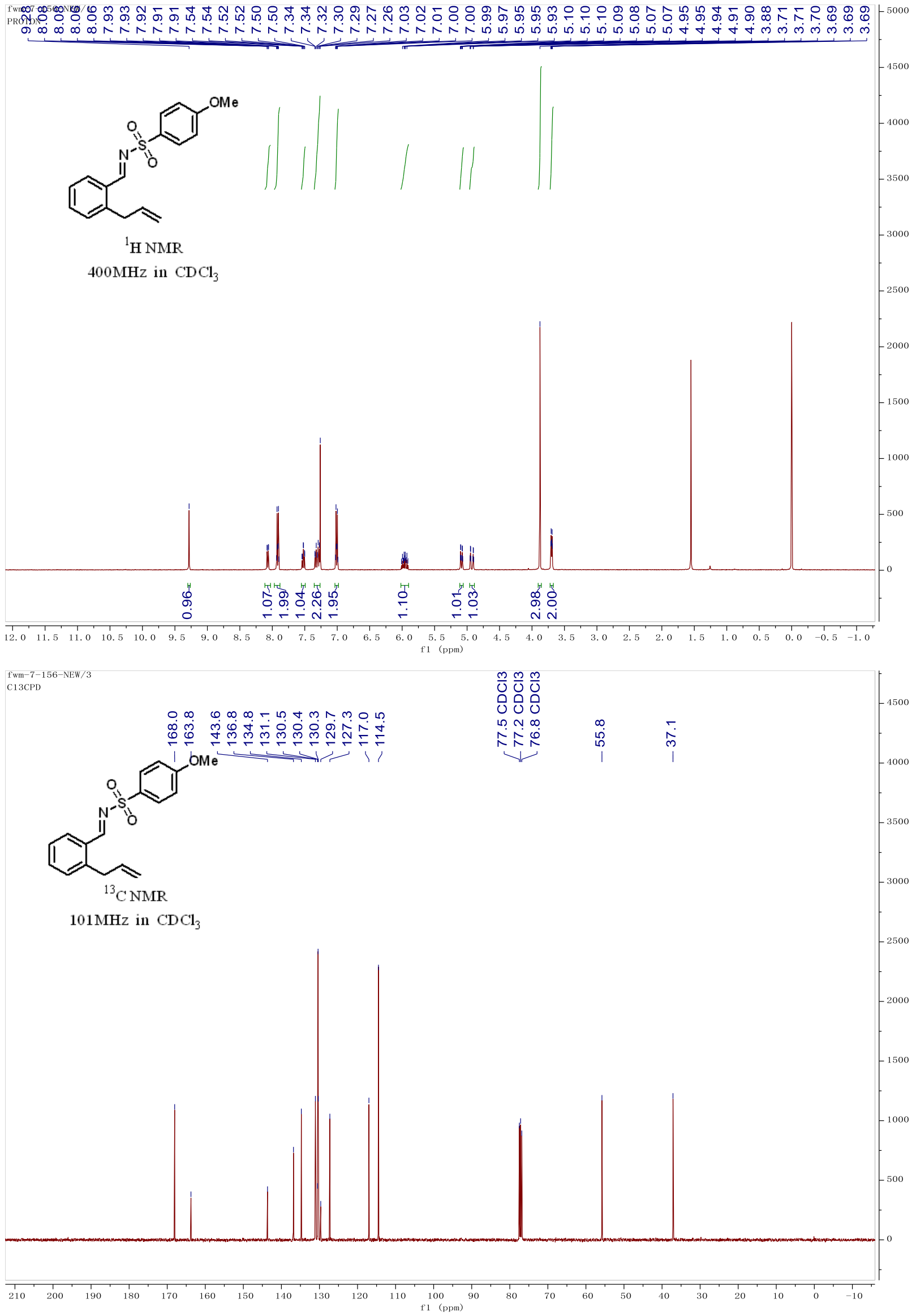


\section{Compound 4d}

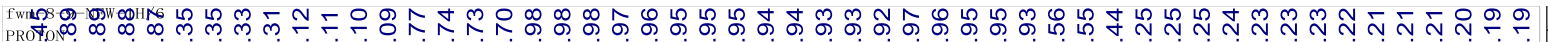

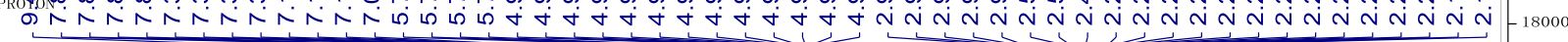

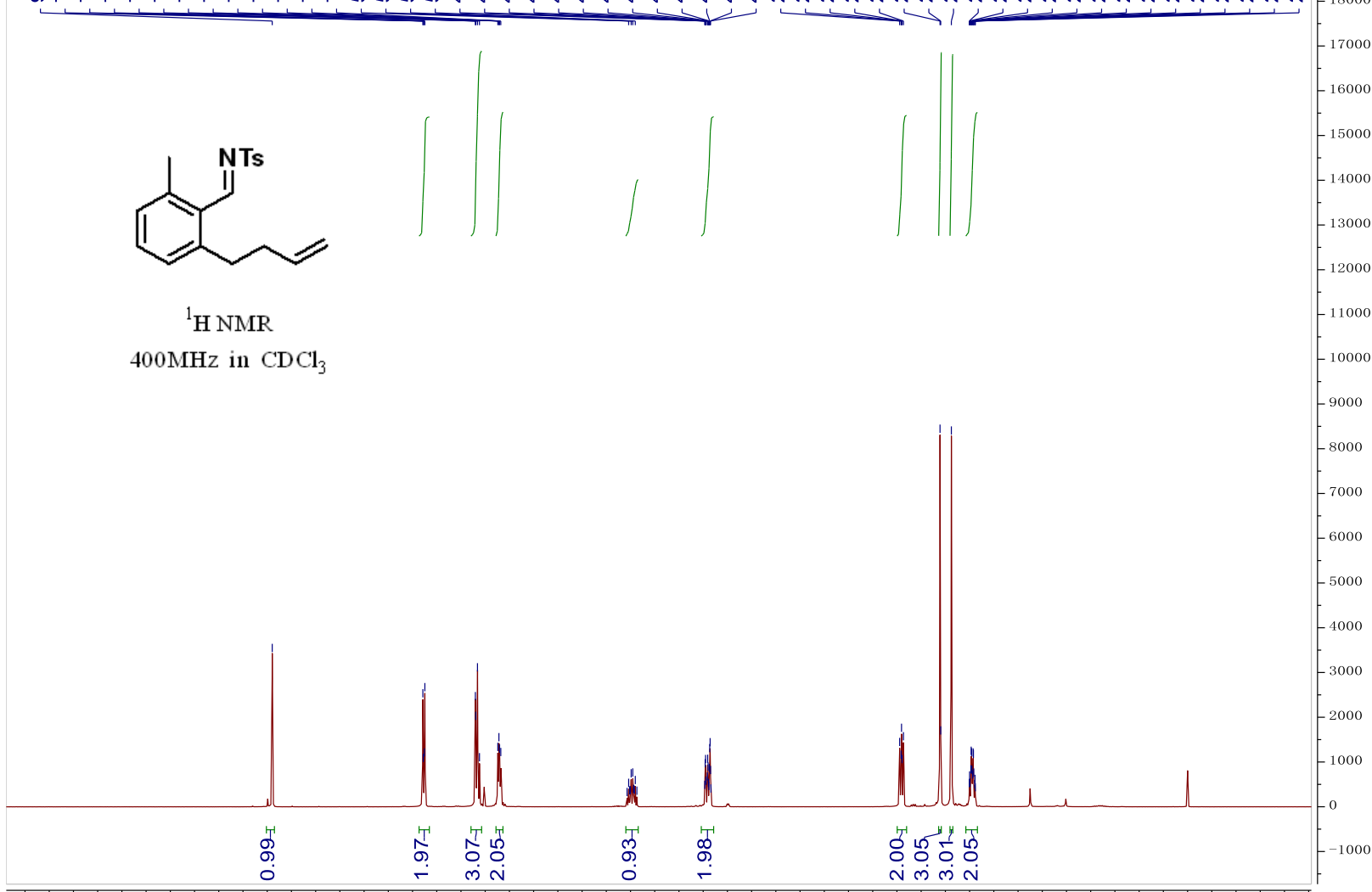

\begin{tabular}{rlllllllllllllllllllllllllllllllll}
\hline 12.0 & 11.5 & 11.0 & 10.5 & 10.0 & 9.5 & 9.0 & 8.5 & 8.0 & 7.5 & 7.0 & 6.5 & 6.0 & 5.5 & 5.0 & 4.5 & 4.0 & 3.5 & 3.0 & 2.5 & 2.0 & 1.5 & 1.0 & 0.5 & 0.0 & -0.5 & -1.0
\end{tabular}

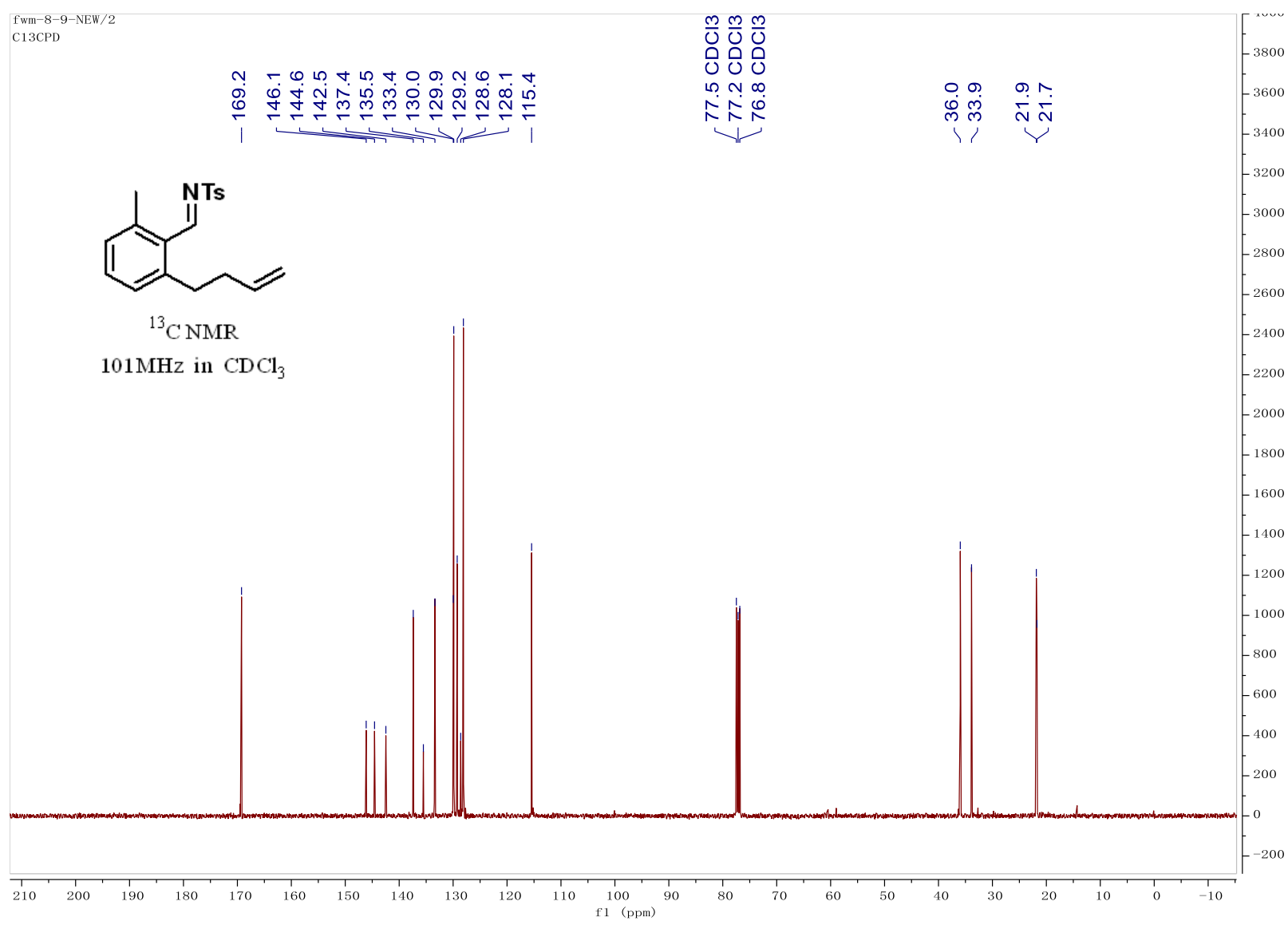




\section{Compound 4e}
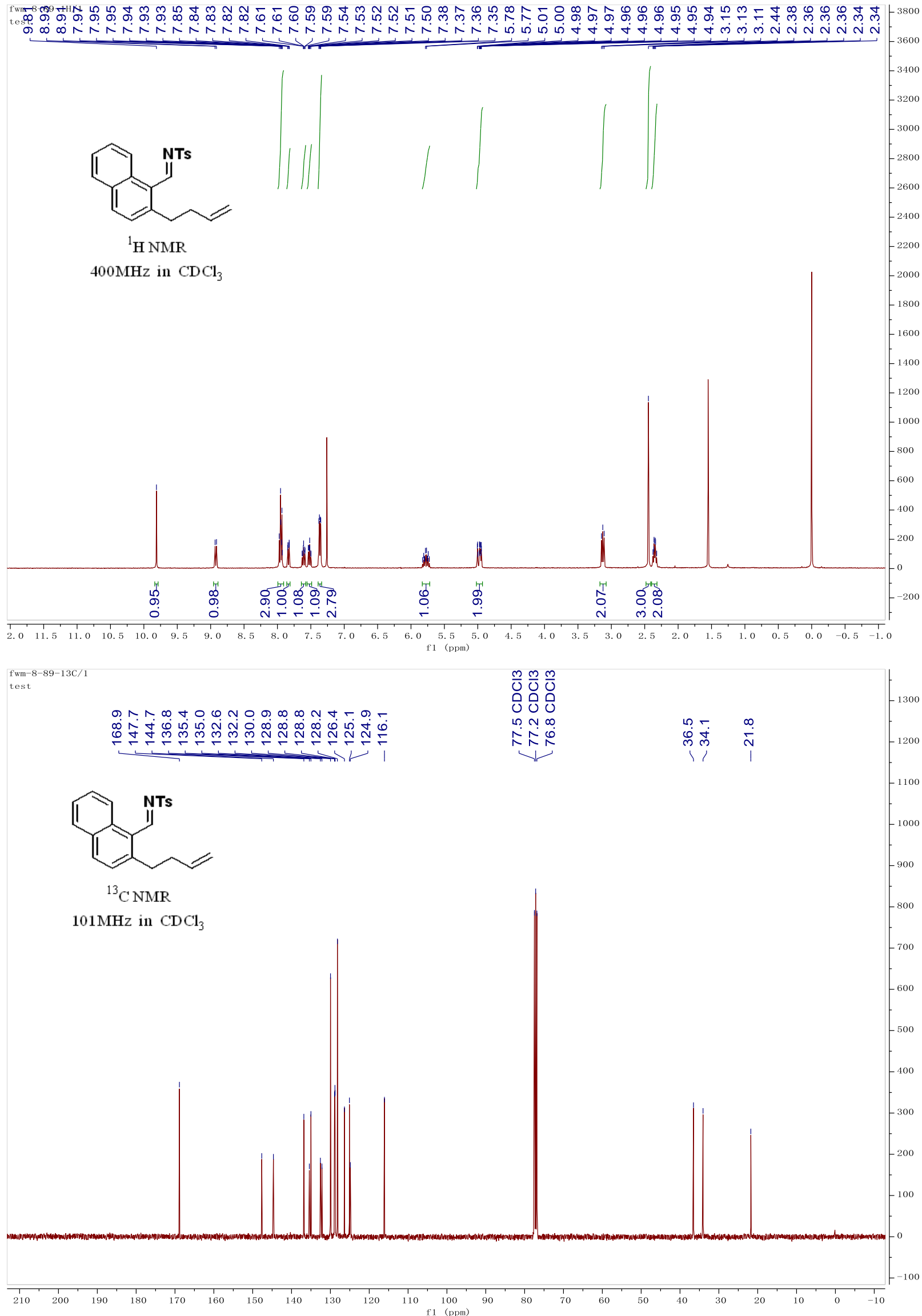


\section{Compound 4h}

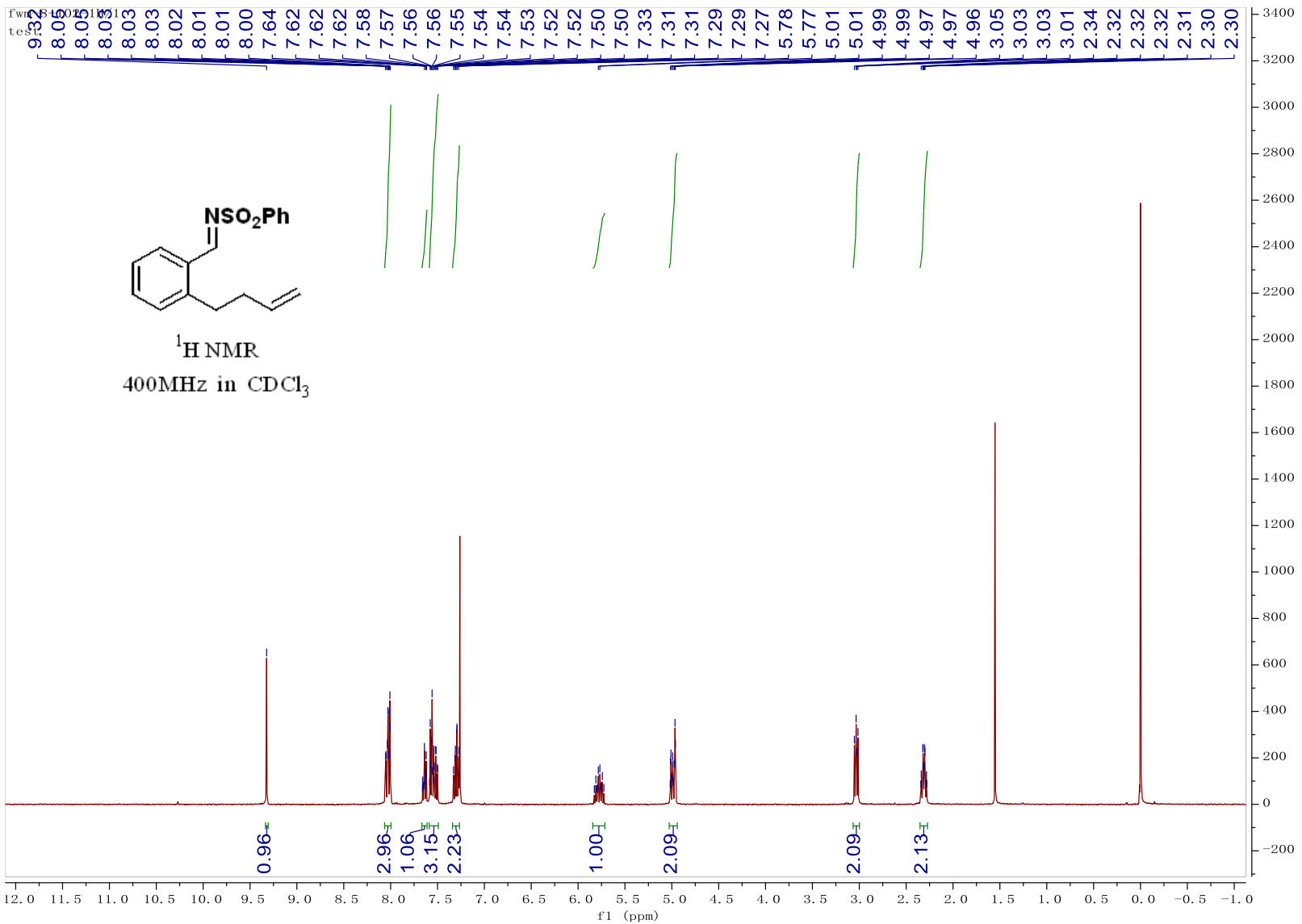

fwm-8-102-13C/2
C13CPD

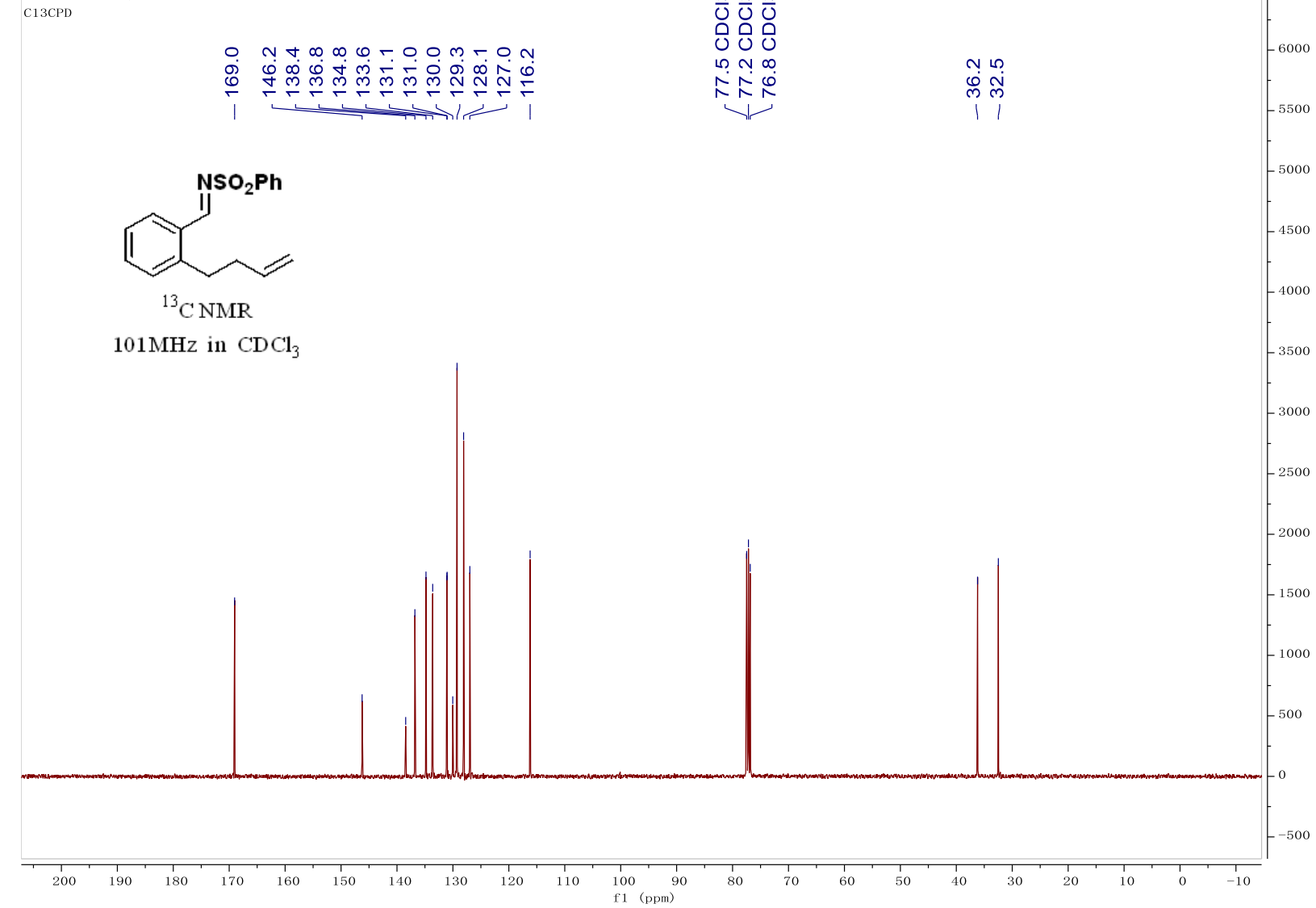




\section{Compound 4i}

fwr

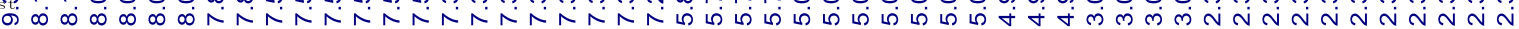
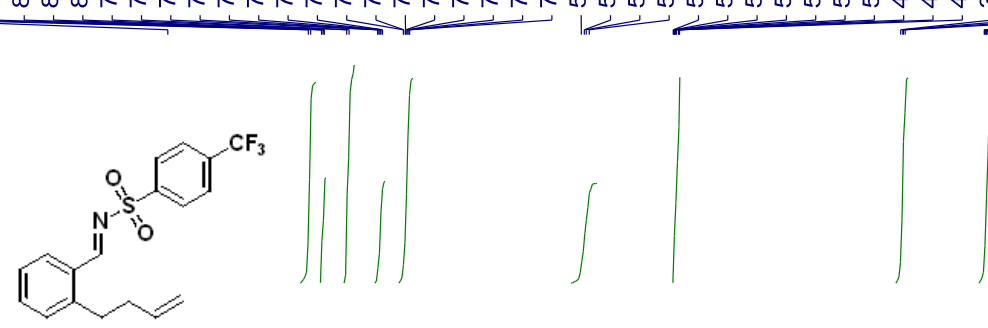

${ }^{1} \mathrm{H}$ NMR

$400 \mathrm{MHz}$ in $\mathrm{CDCl}_{3}$
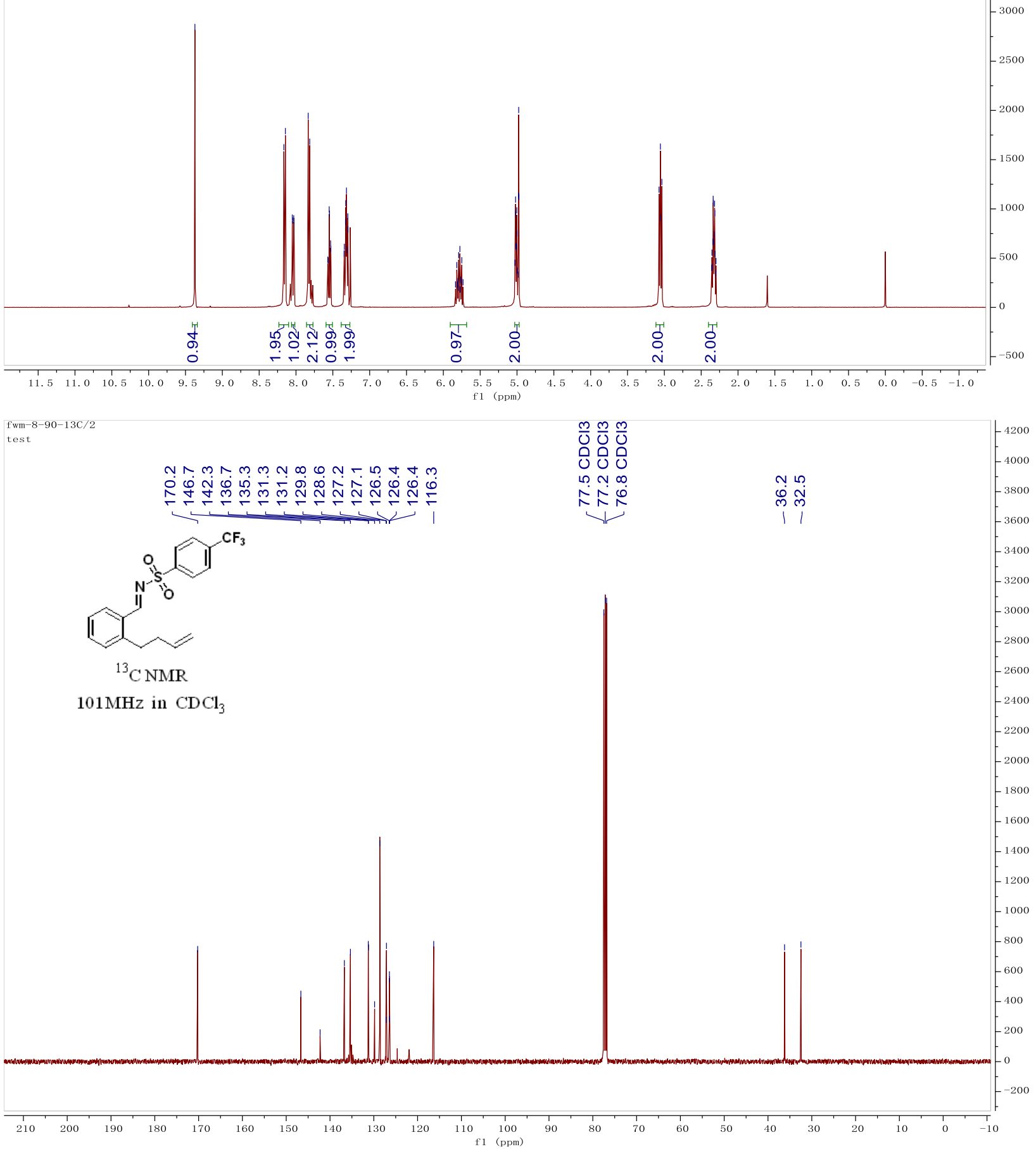


\section{Compound $4 \mathbf{j}$}

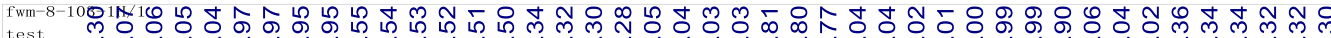

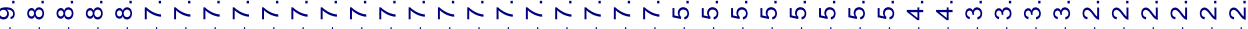
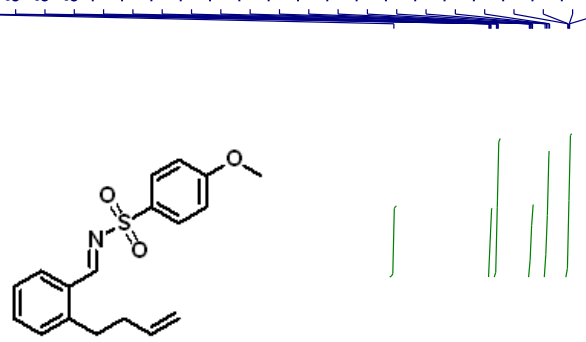

${ }^{1} \mathrm{H}$ NMR

$400 \mathrm{MHz}$ in $\mathrm{CDCl}_{3}$
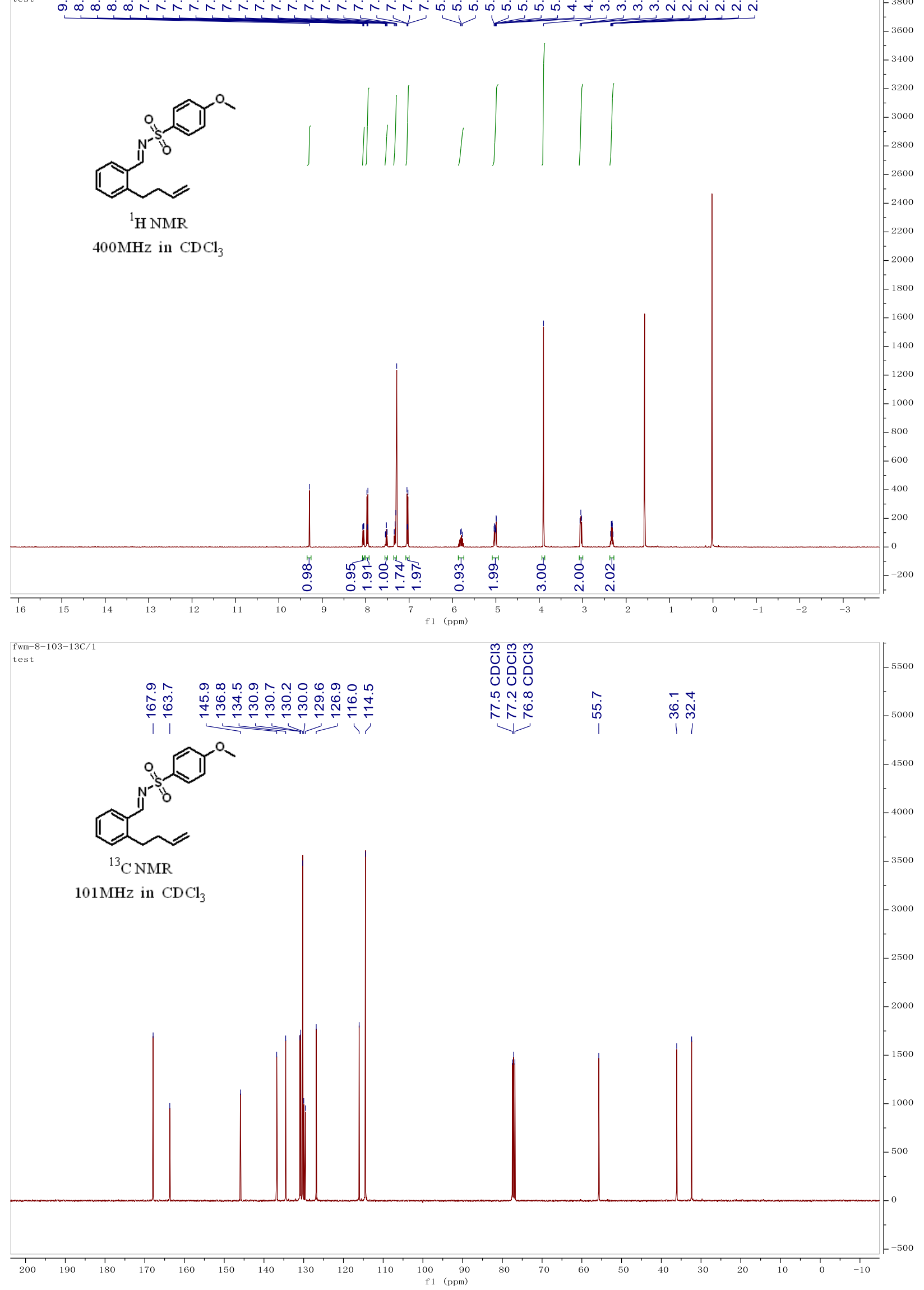


\section{Compound 2a}

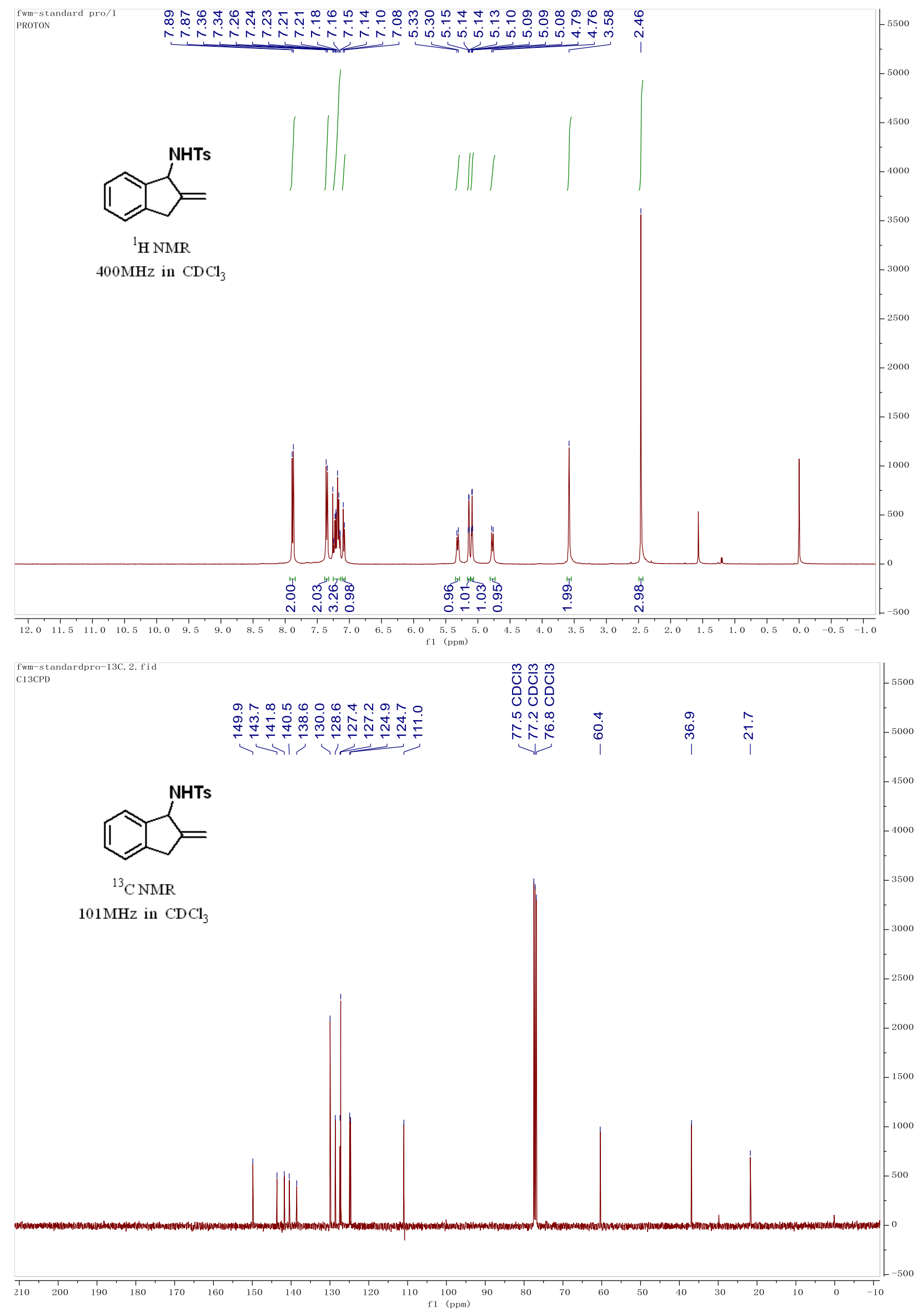




\section{Compound 2b}
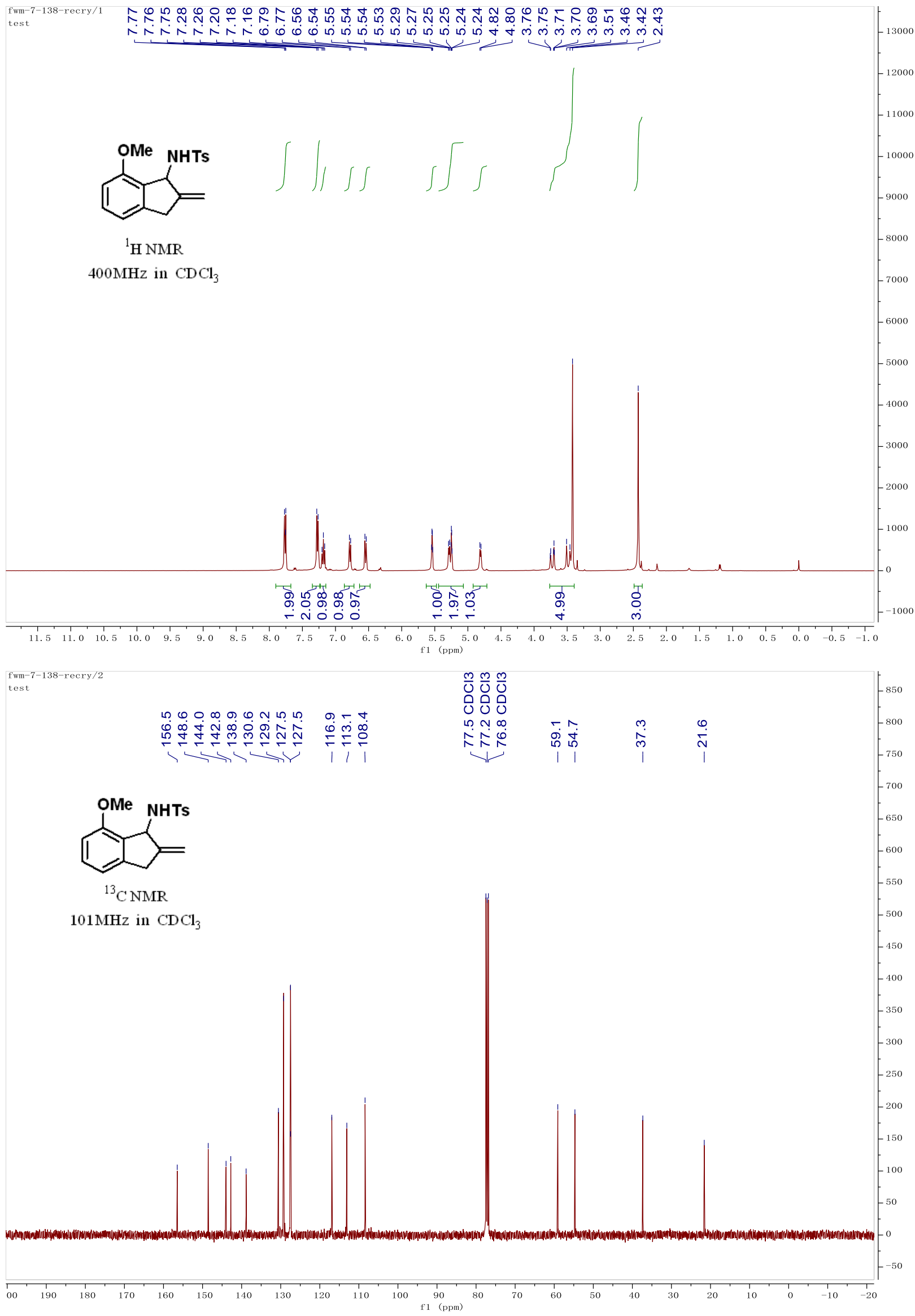


\section{Compound 2c}
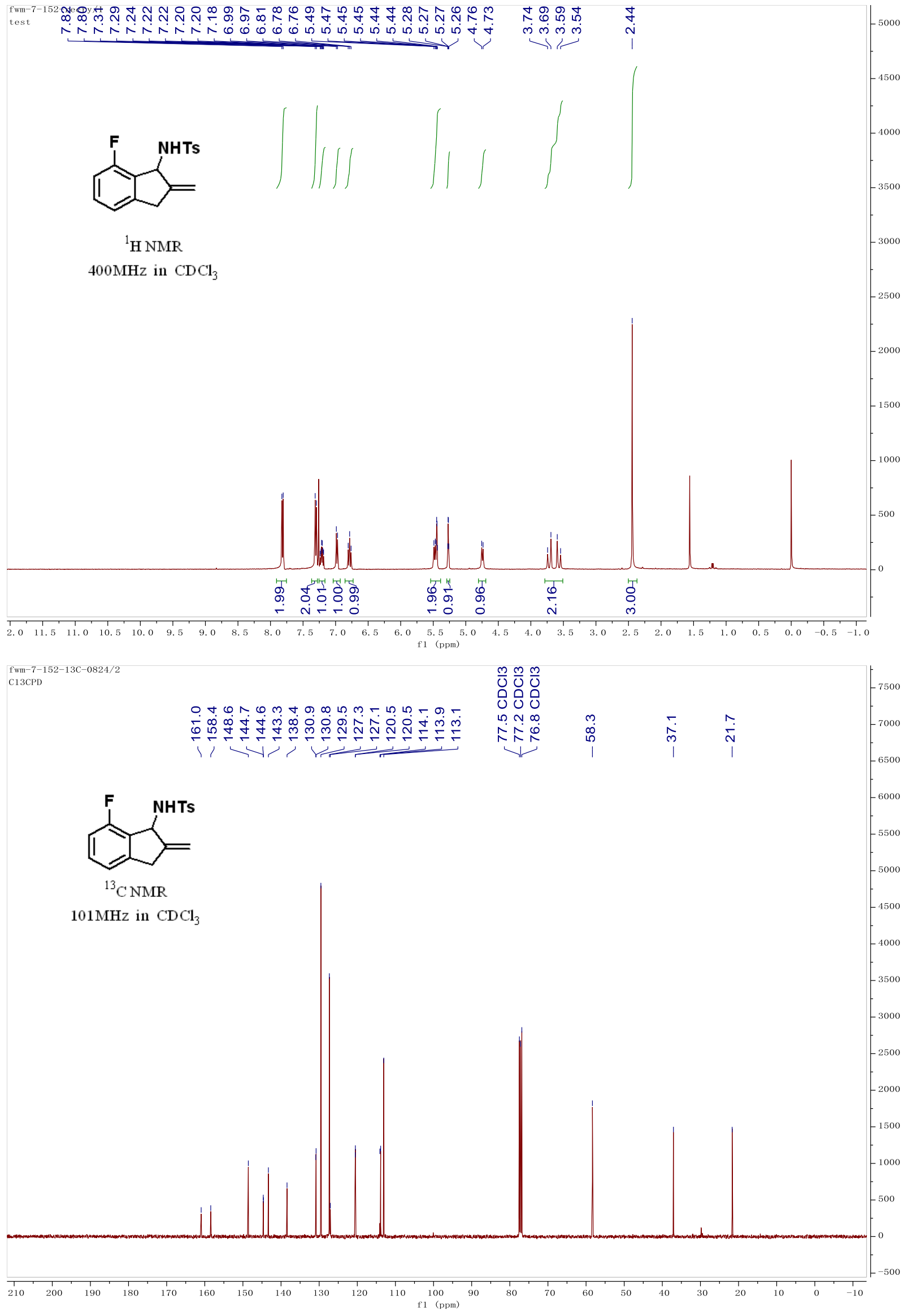


\section{Compound 2d}
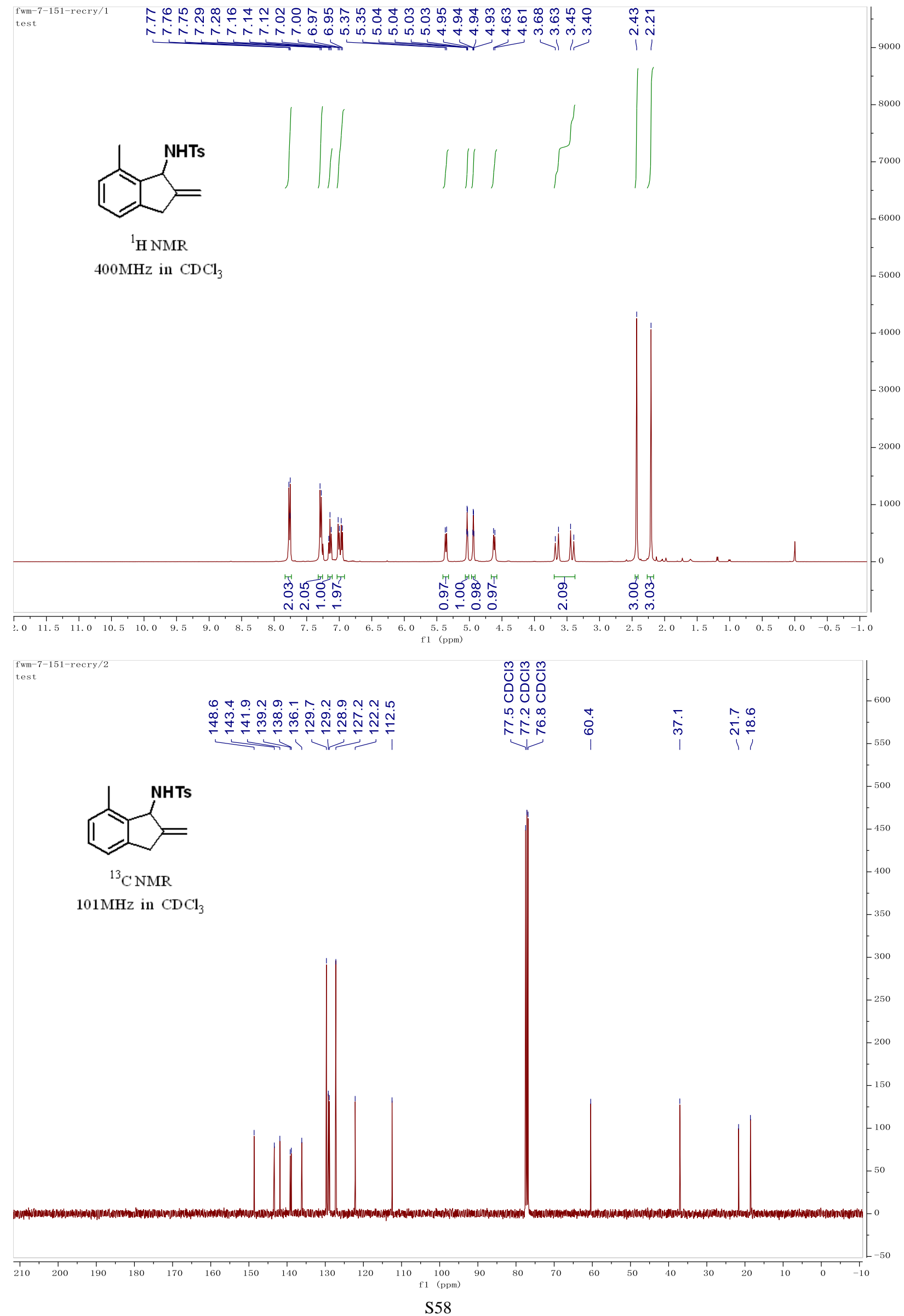


\section{Compound 2e}
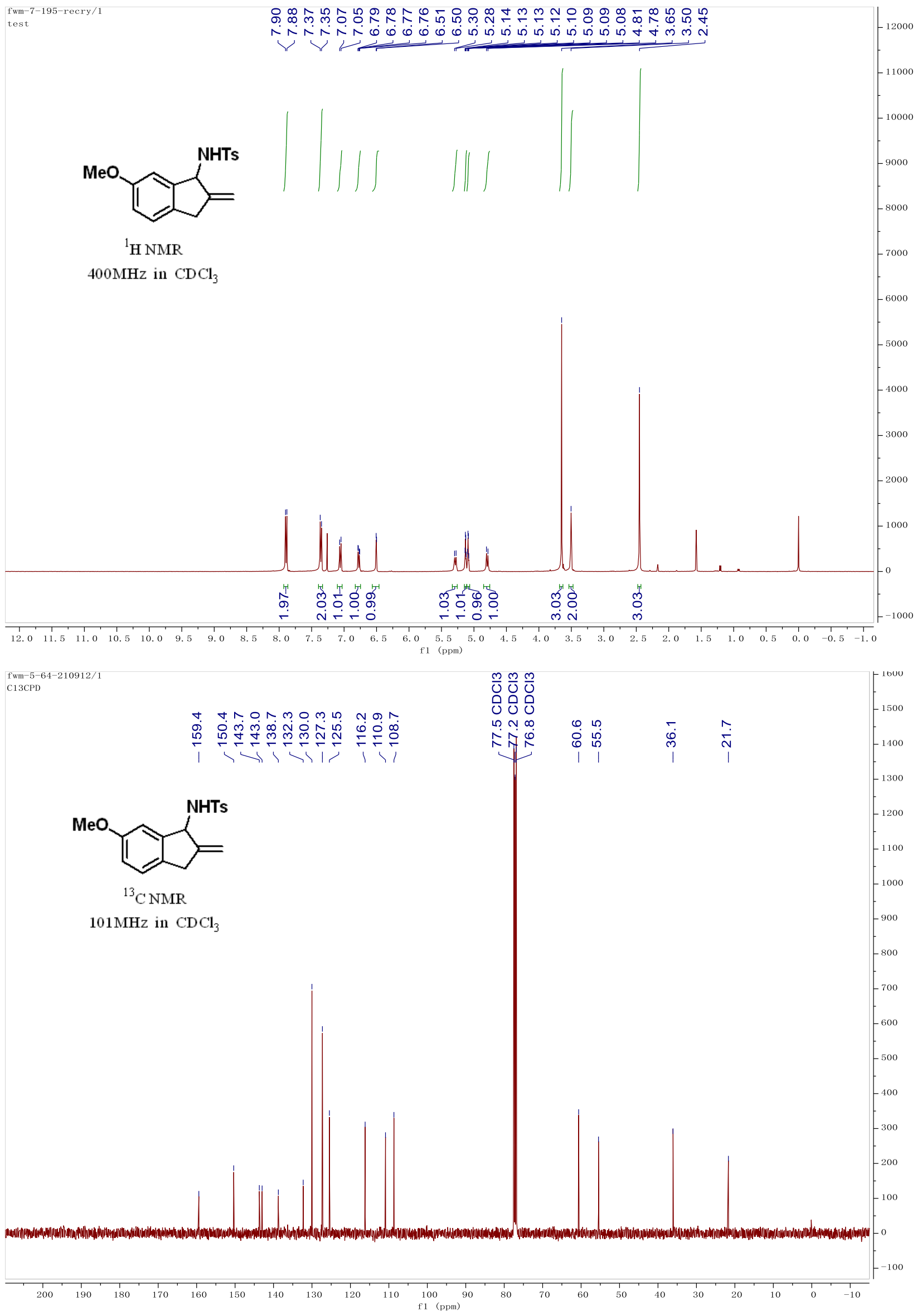


\section{Compound 2f}

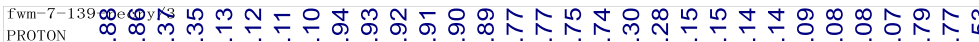

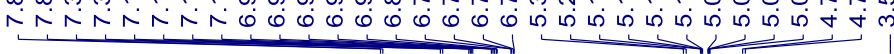<smiles>C=C1Cc2ccc(F)cc2C1NC(F)(F)F</smiles>

${ }^{1} \mathrm{H}$ NMR

$400 \mathrm{MHz}$ in $\mathrm{CDCl}_{3}$

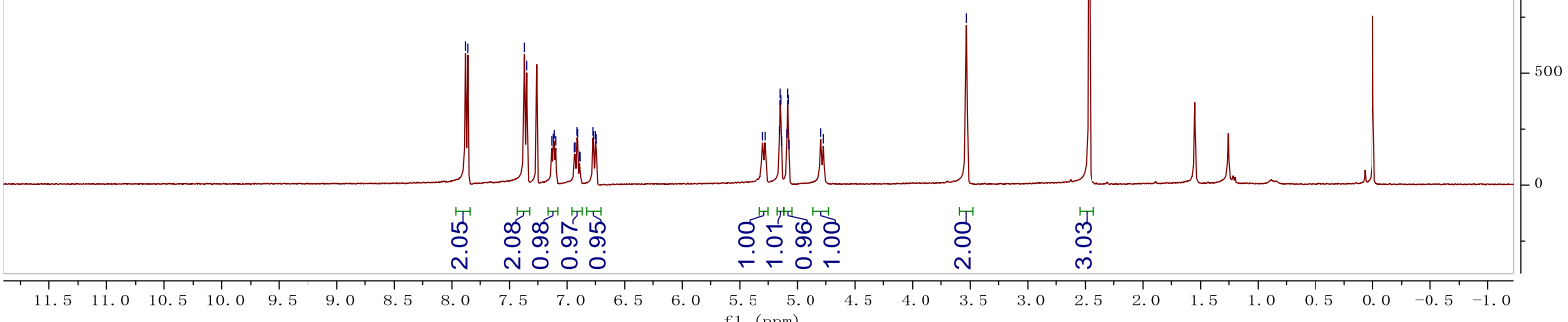
fwm-5-66-210912. 1. fid

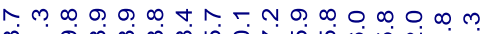

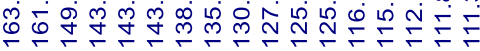

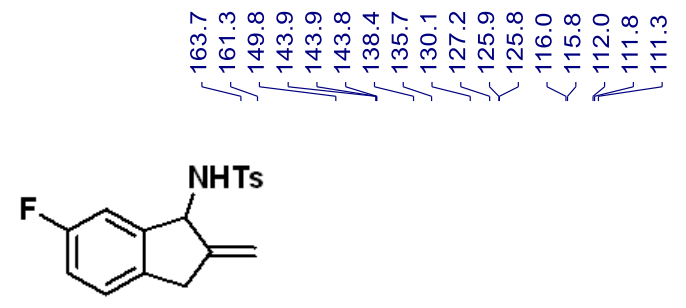

${ }^{13} \mathrm{CNMR}$

$101 \mathrm{MHz}$ in $\mathrm{CDCl}_{3}$ 5. 5 (1) fwm $-5-66-2$
C13CPD

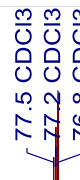

\section{$\frac{m}{0} \frac{m}{0}$} in $\mathrm{N}$ 舟 Nํㅇ $\stackrel{+}{\circ}$ $\stackrel{N}{\stackrel{N}{n}}$

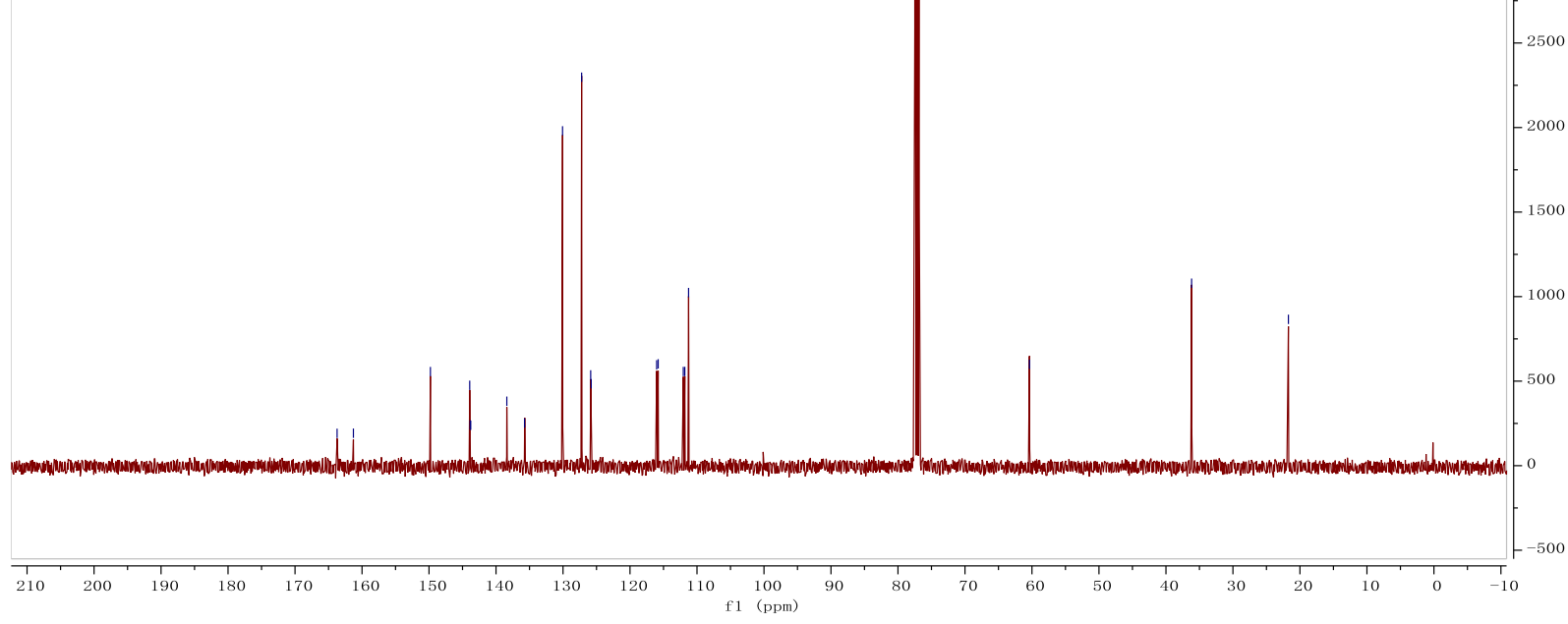




\section{Compound 2g}

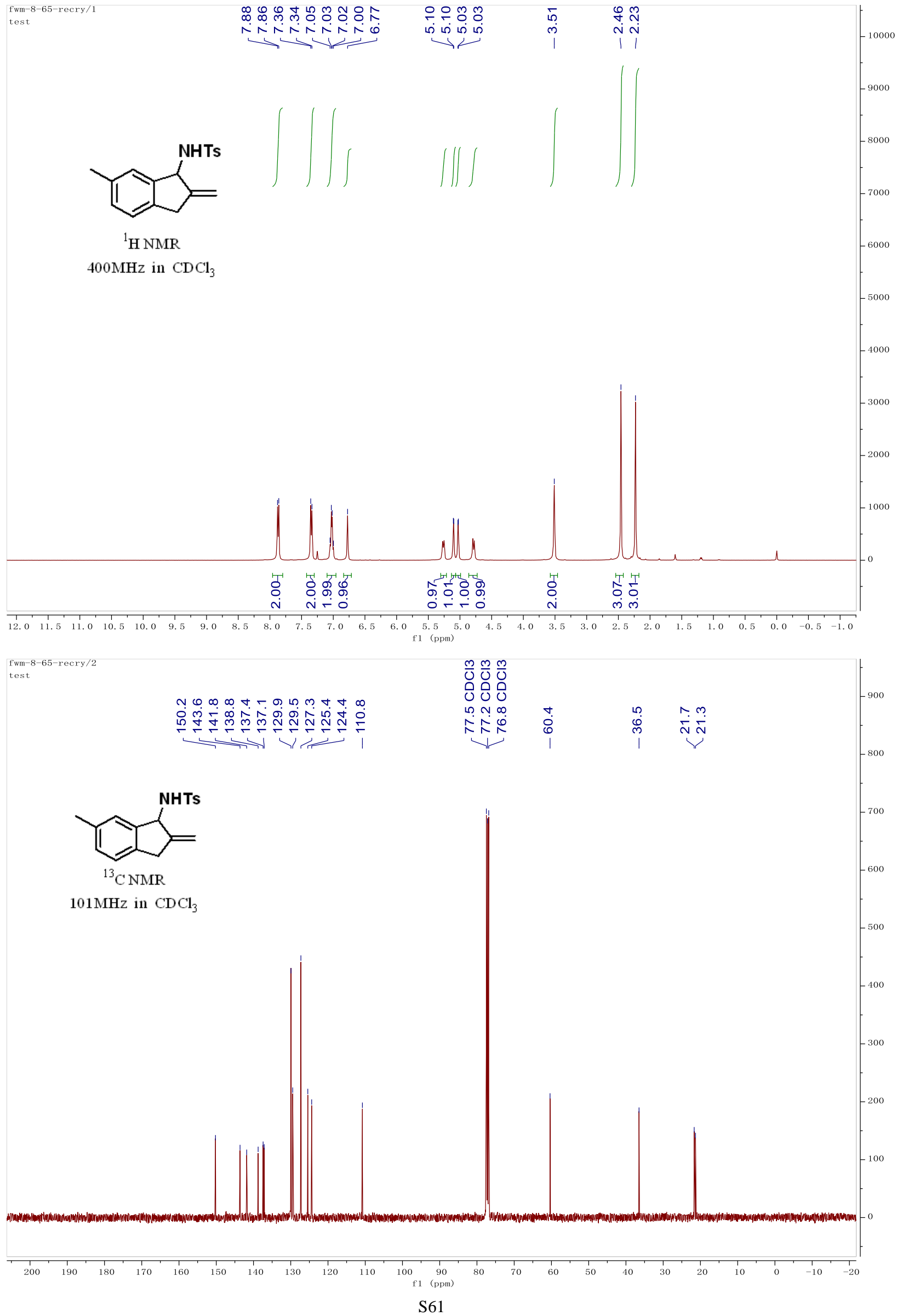




\section{Compound 2h}
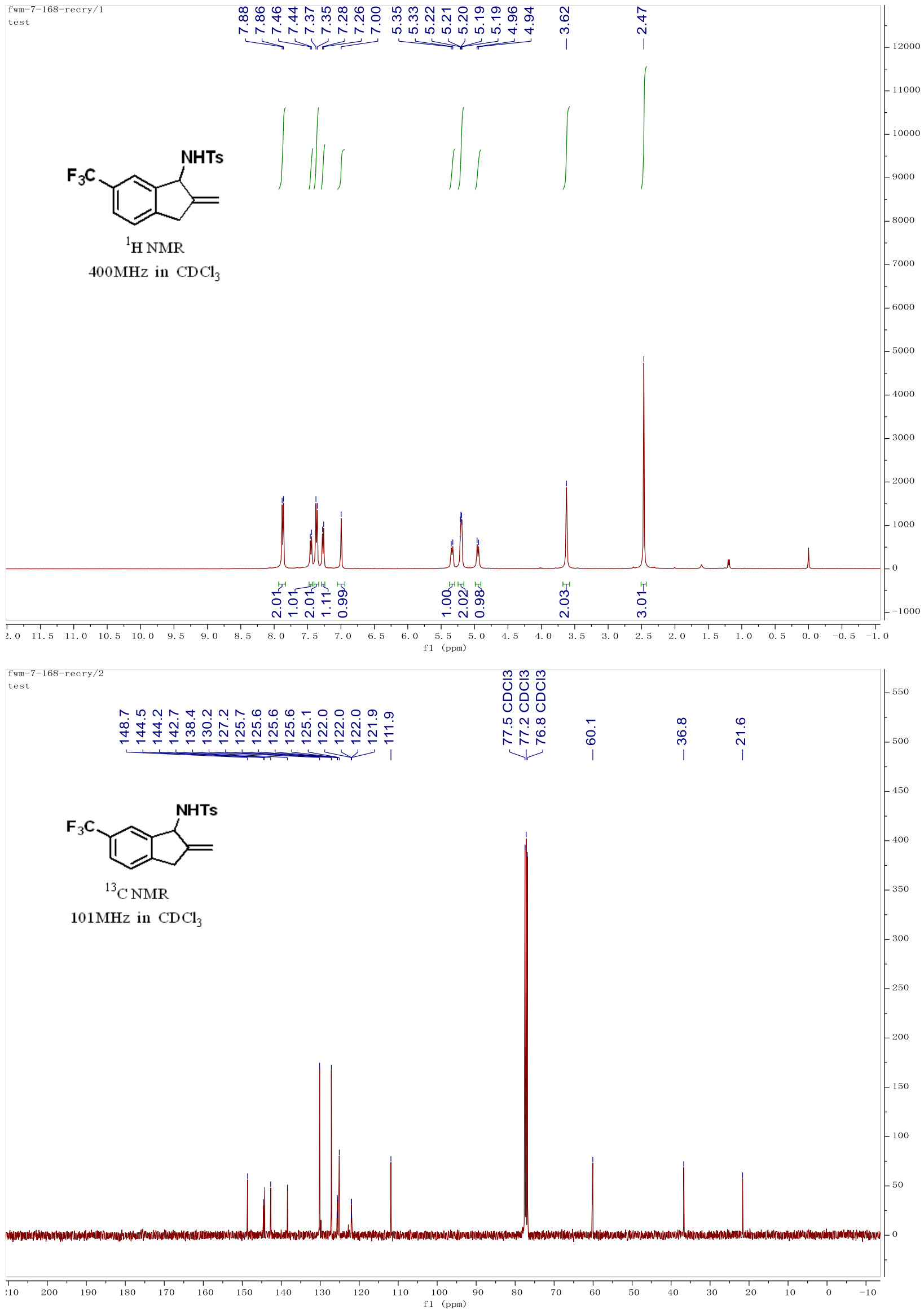


\section{Compound 2i}
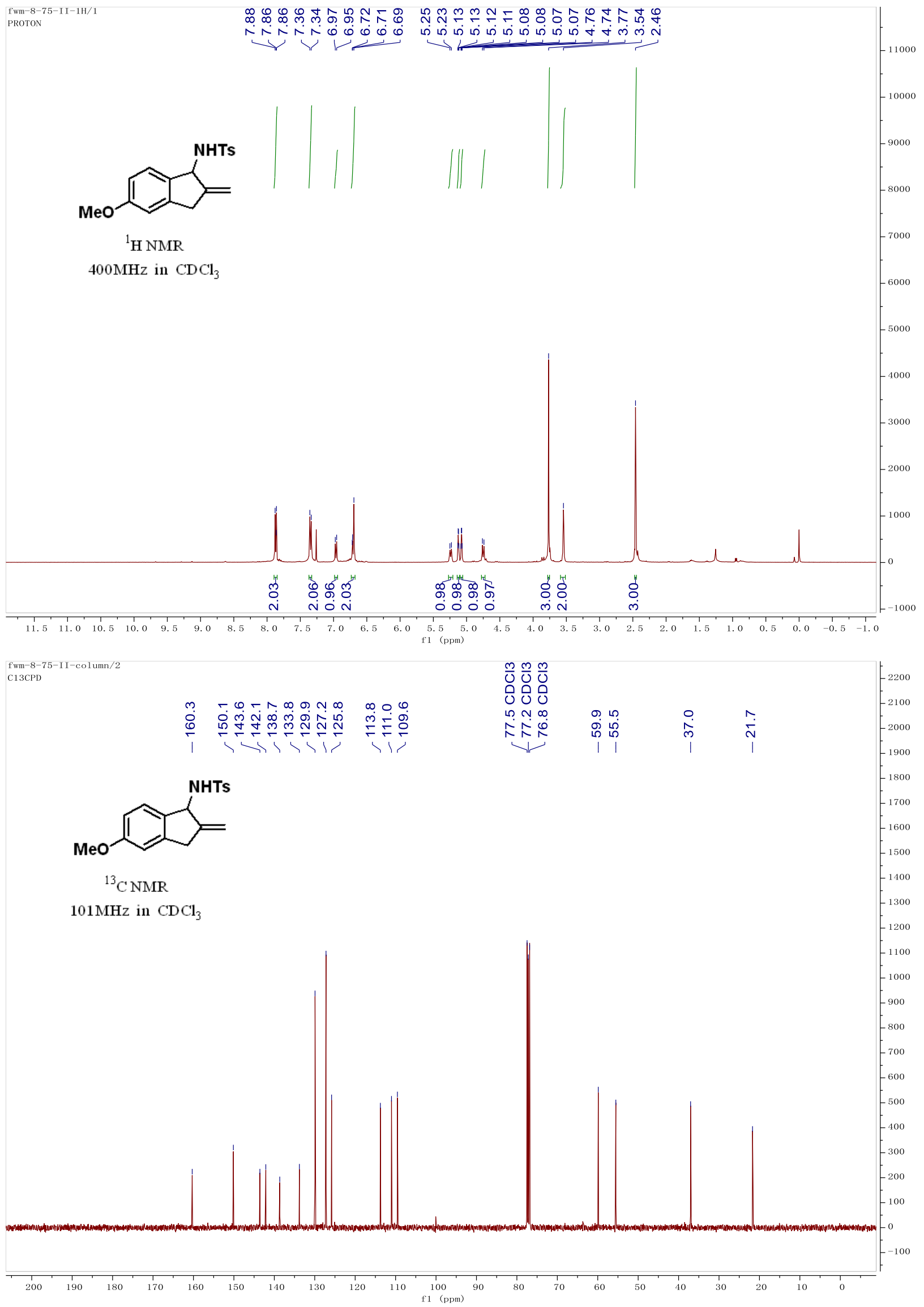


\section{Compound 2j}

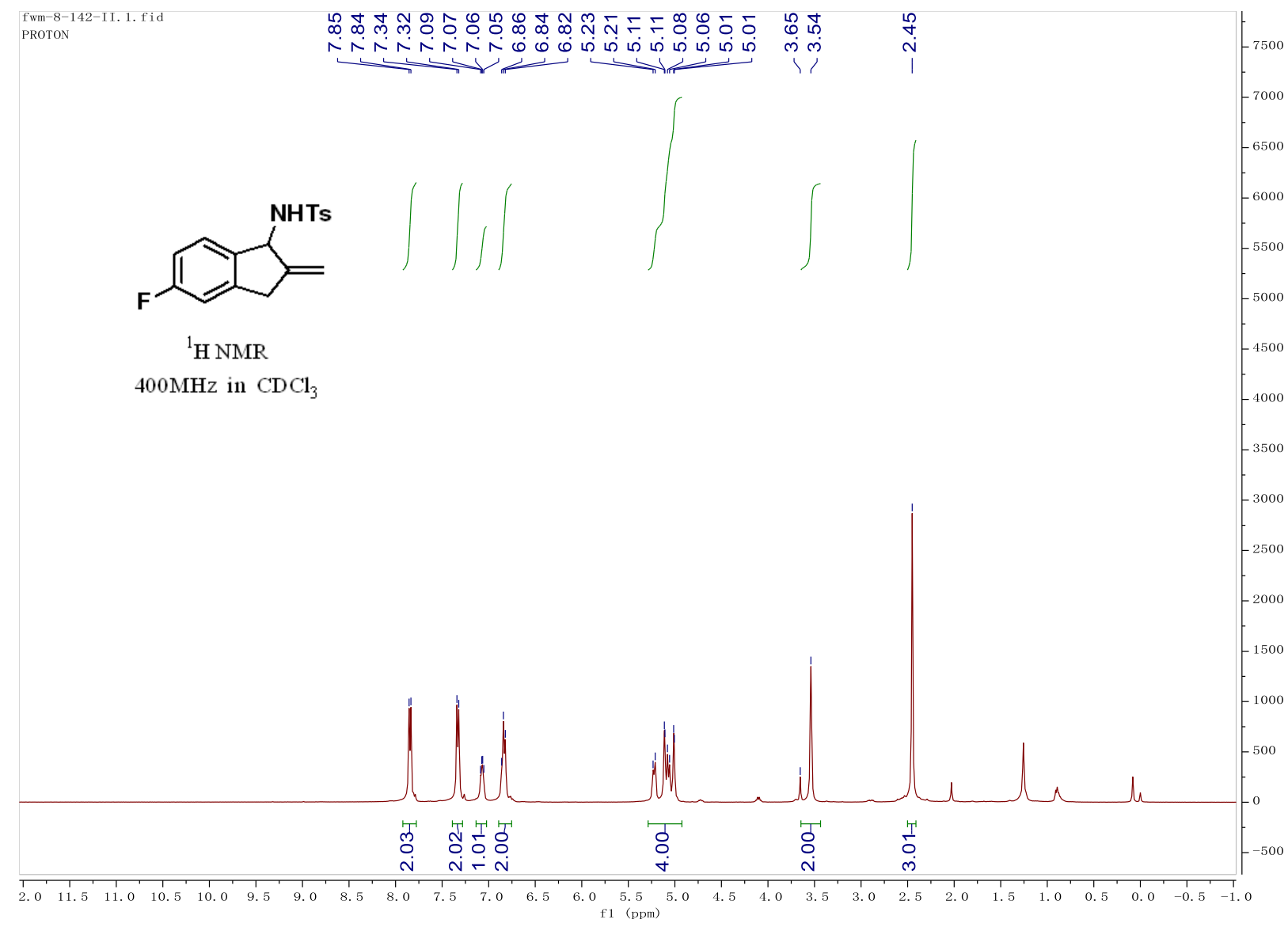

fwm-8-142-II. 2. fid C13CPD

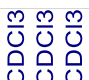

บํำ

穴穴

ஸ்

$\stackrel{\infty}{\dot{0}}$

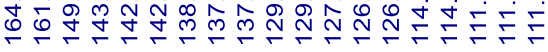

证

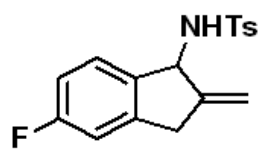

${ }^{13} \mathrm{C}$ NMR

$101 \mathrm{MHz}$ in $\mathrm{CDCl}_{3}$

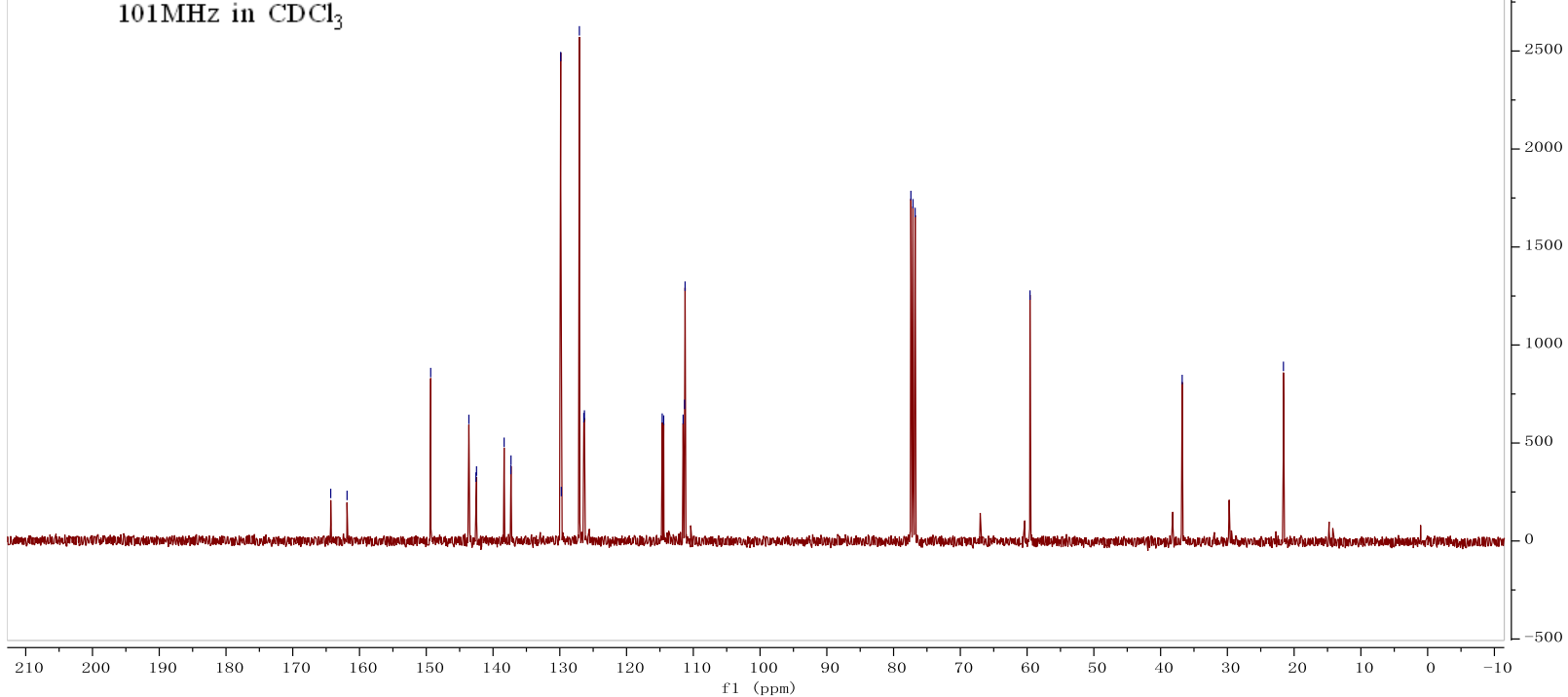




\section{Compound 2k}

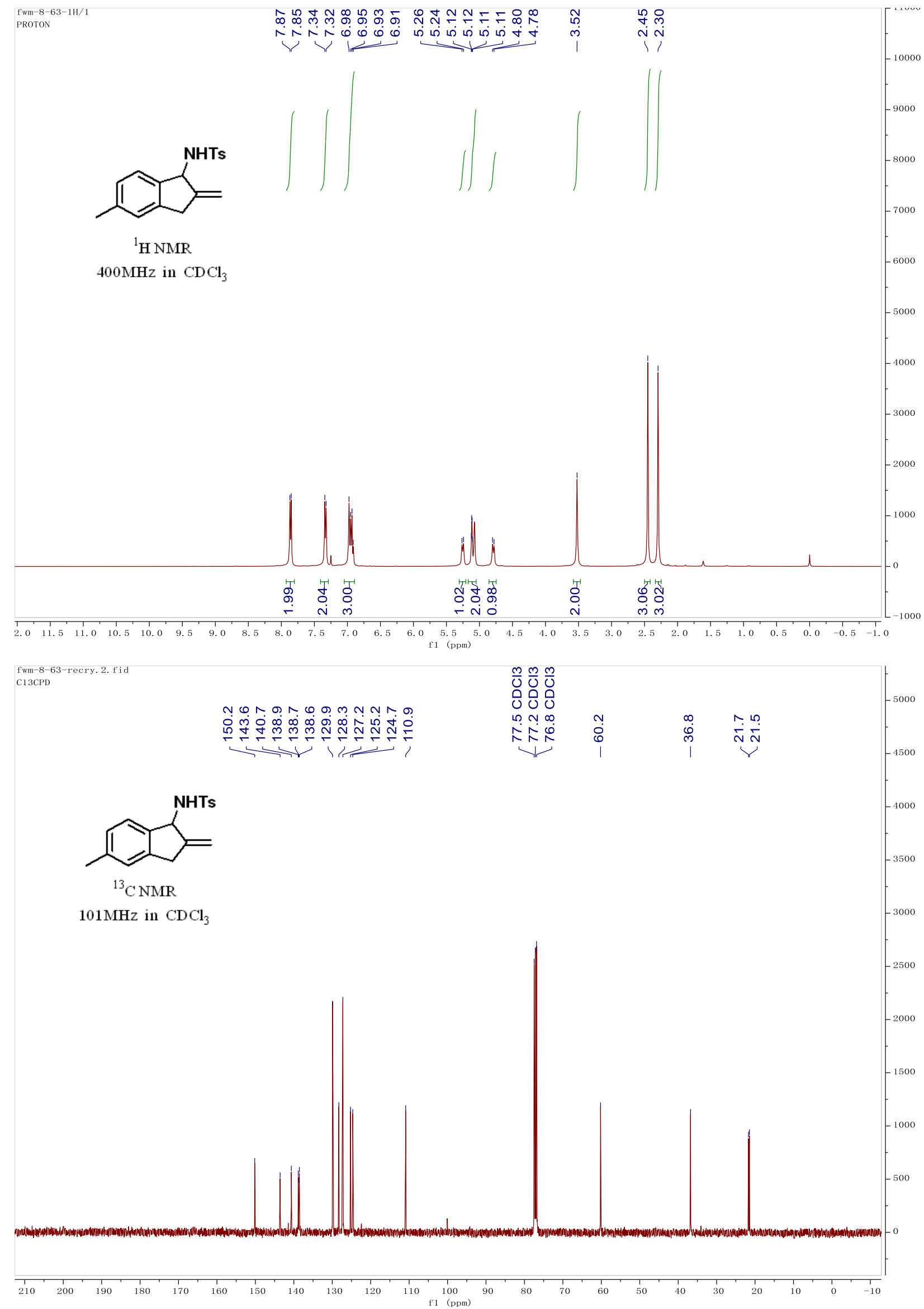




\section{Compound 2l}
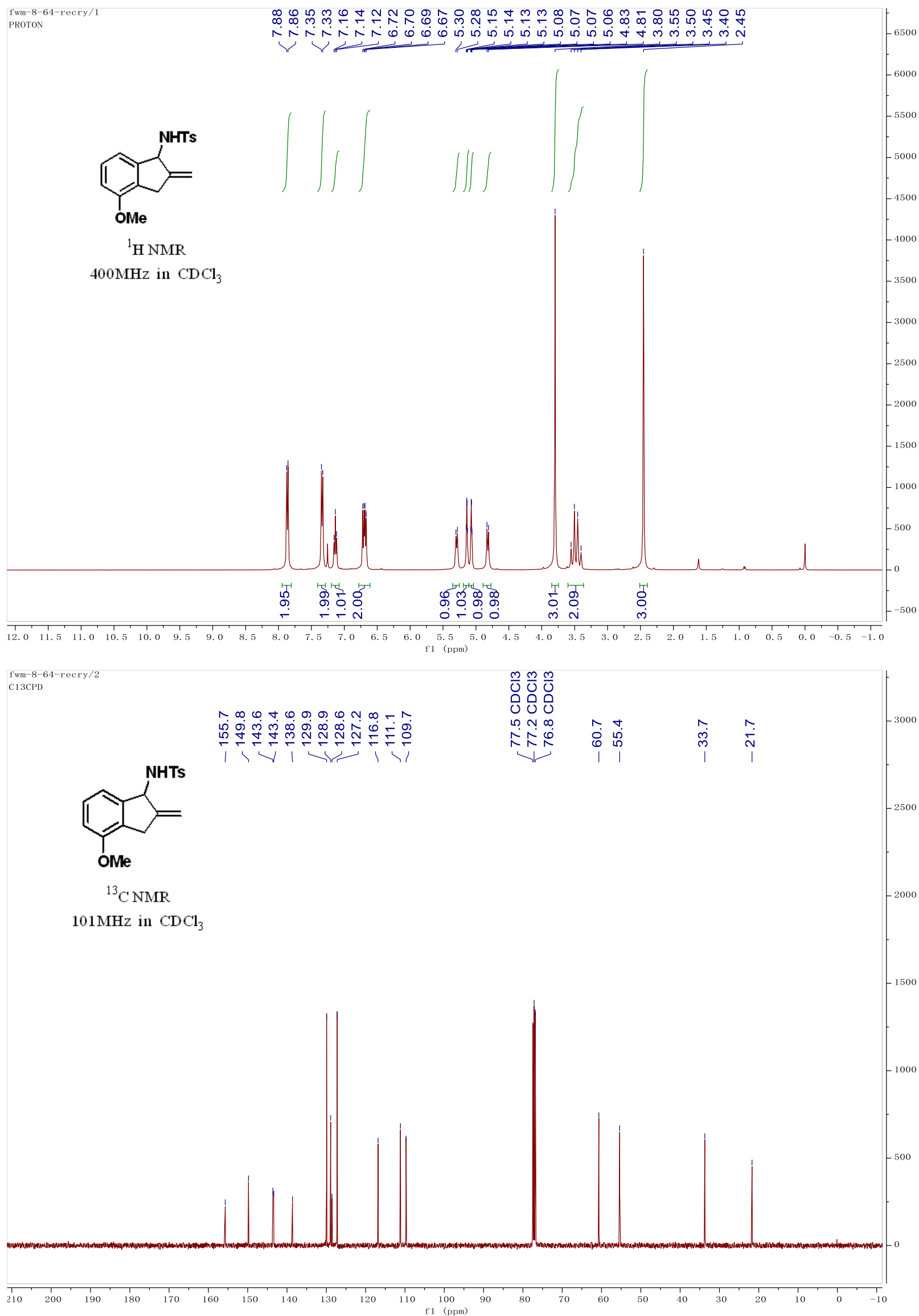


\section{Compound 2m}

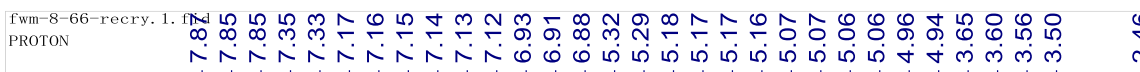
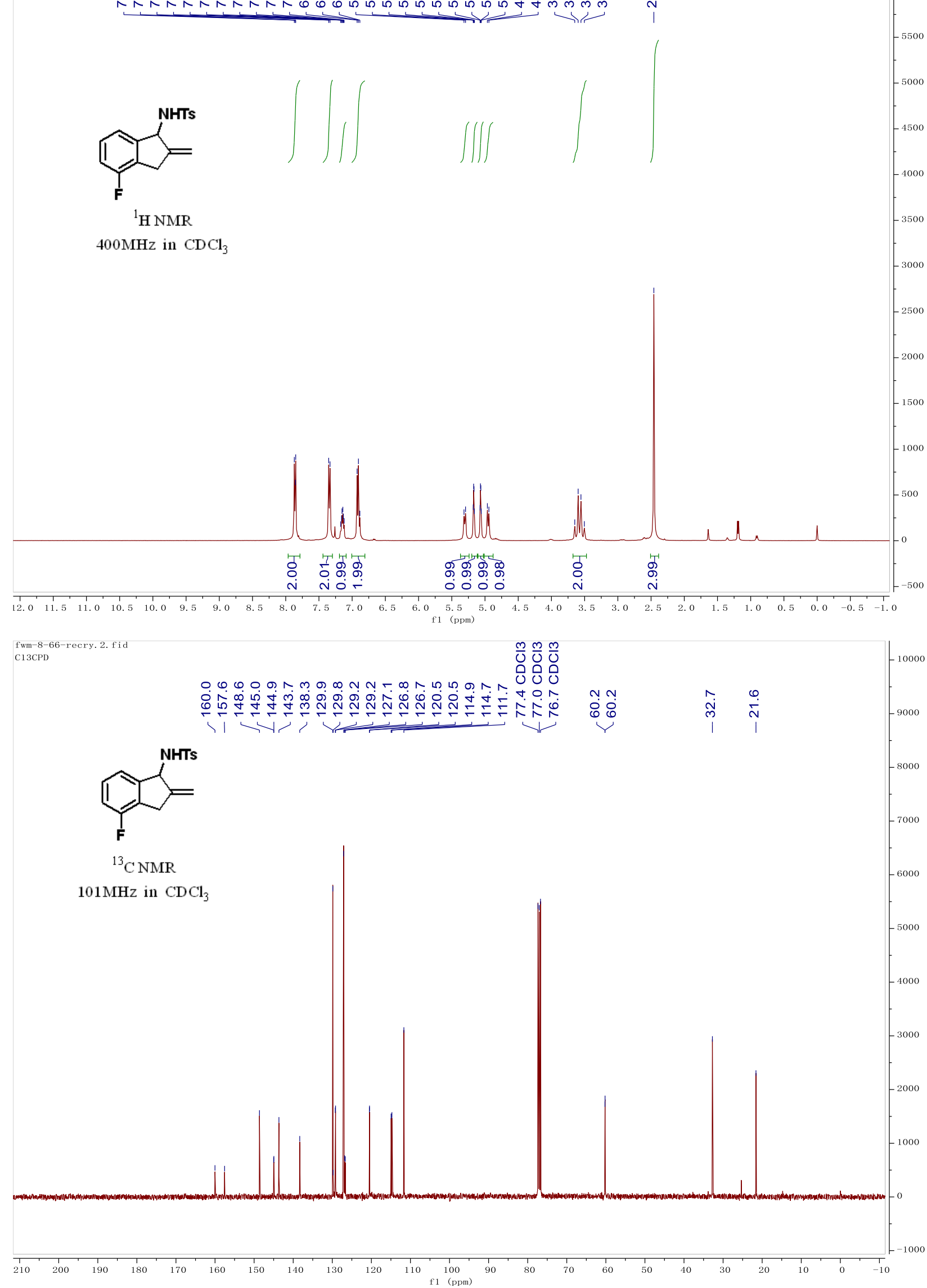


\section{Compound 2n}
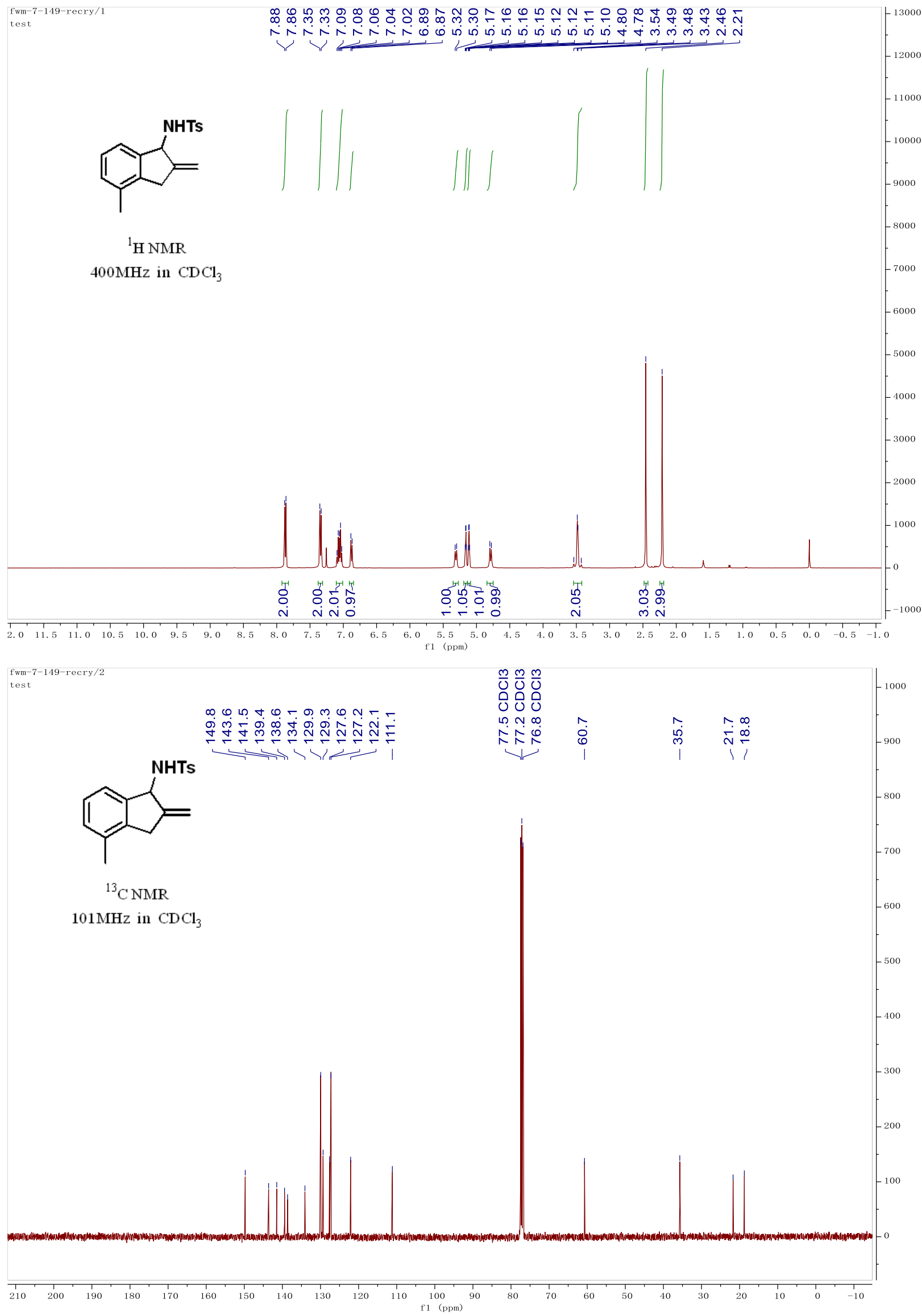


\section{Compound 20}

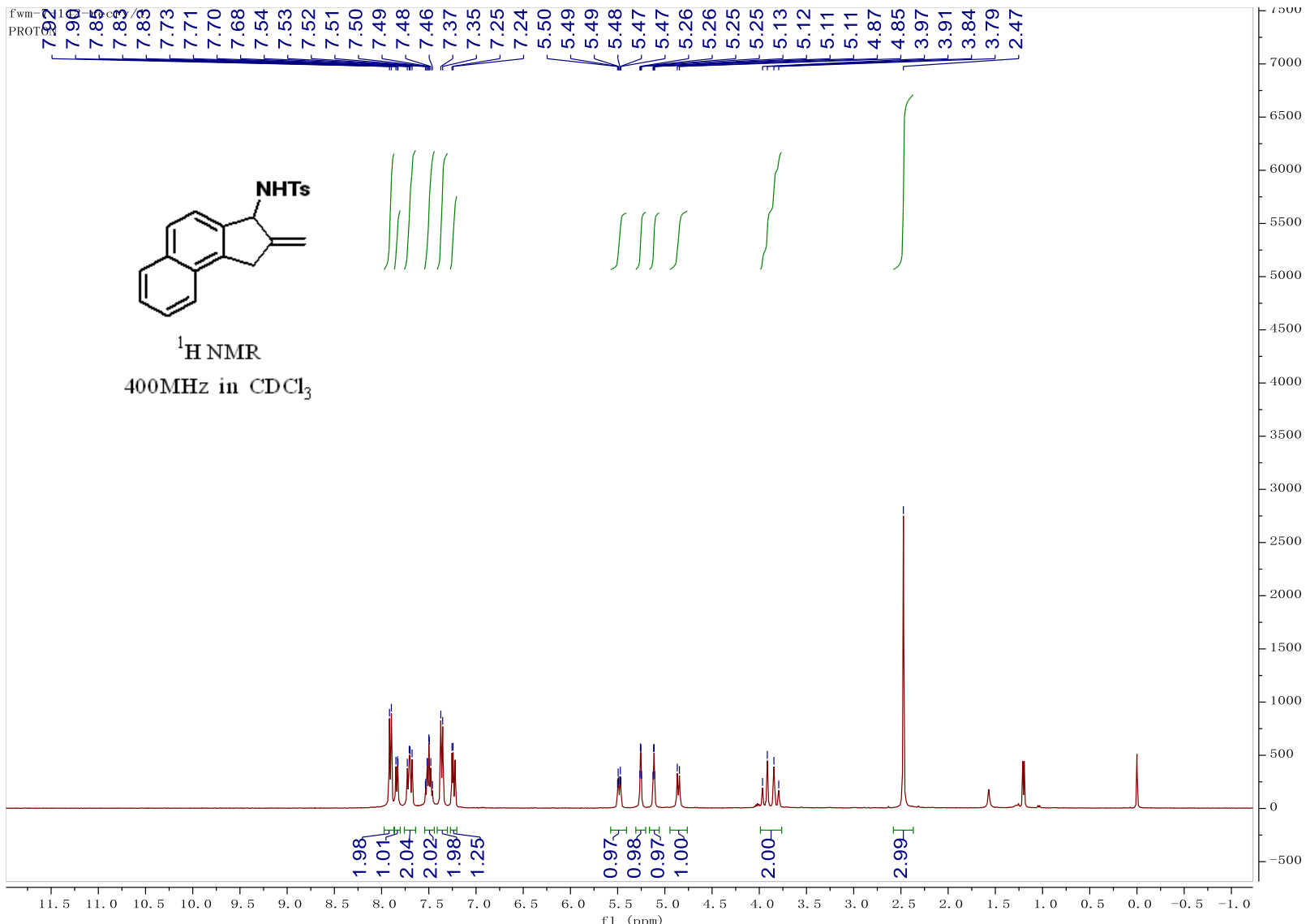

fwm-7-142-recry/2
C13CPD

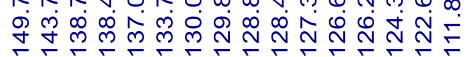
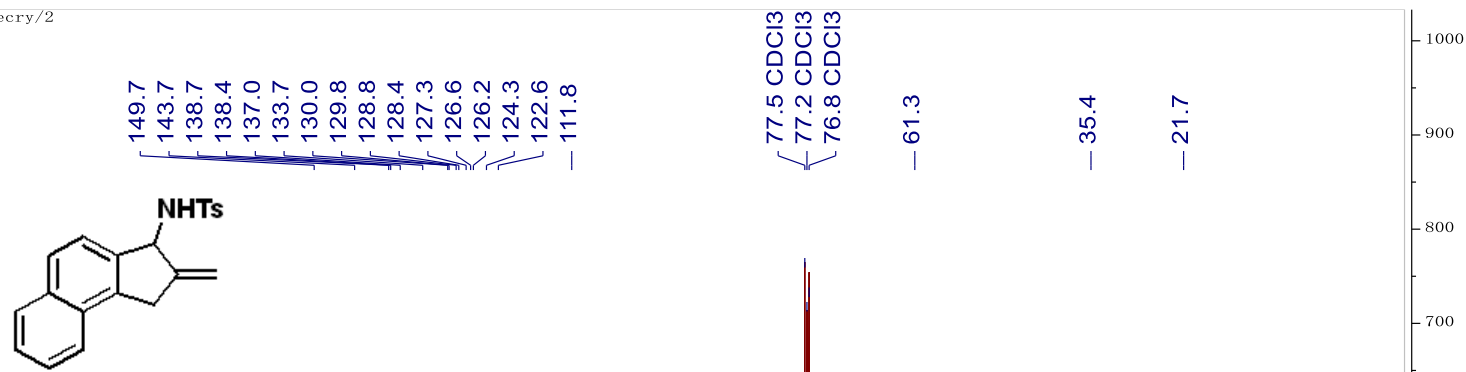

${ }^{13} \mathrm{C}$ NMR

$101 \mathrm{MHz}$ in $\mathrm{CDCl}_{3}$

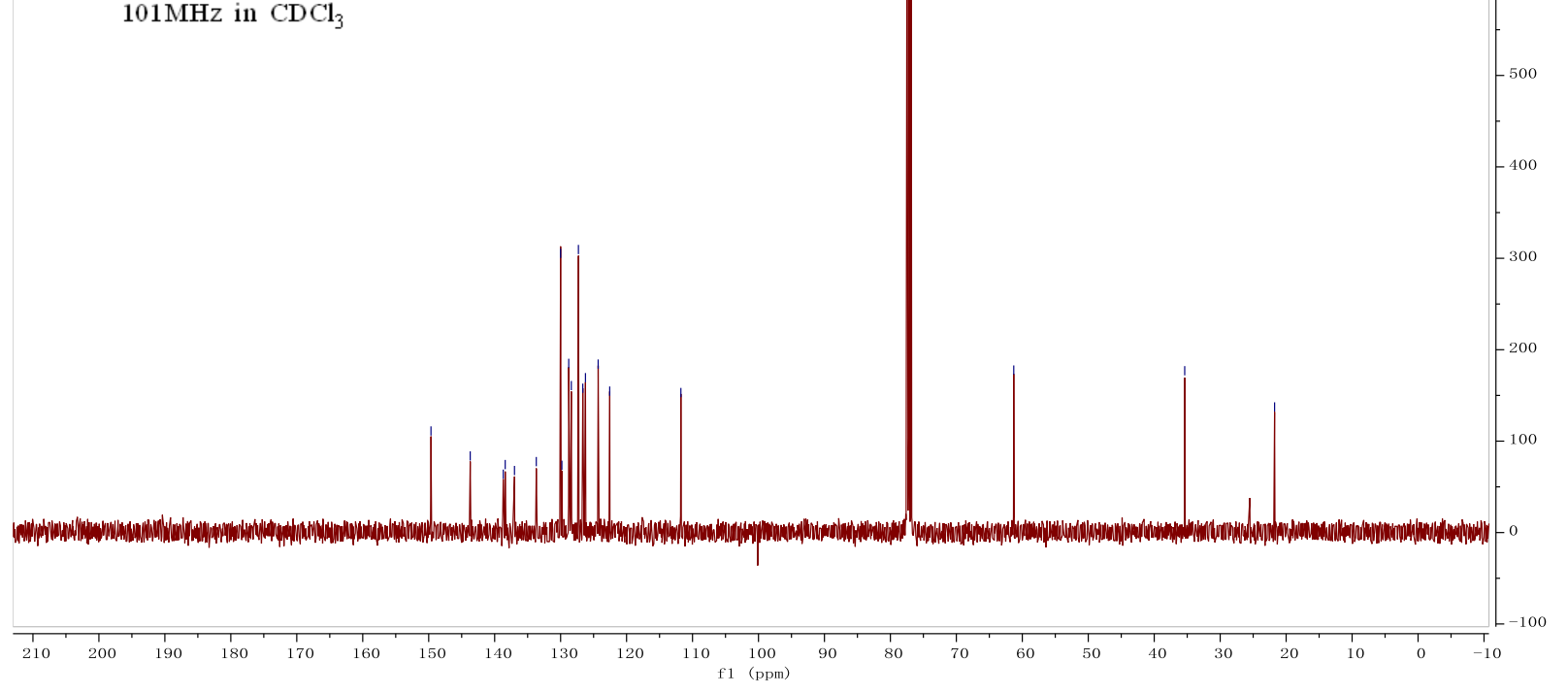




\section{Compound 2p}

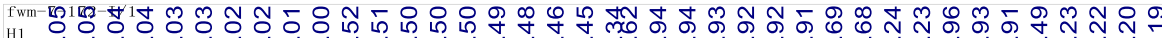

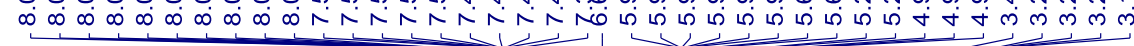

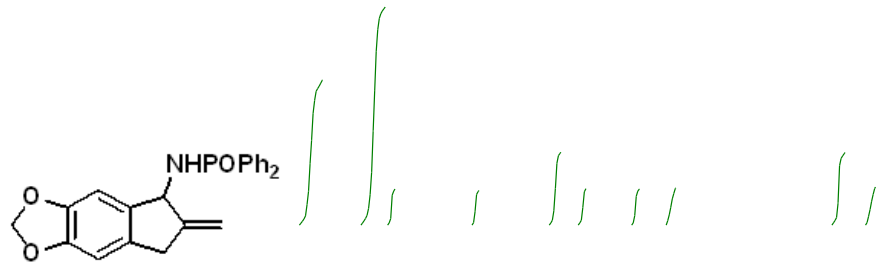

${ }^{1} \mathrm{H} \mathrm{NMR}$

$400 \mathrm{MHz}$ in $\mathrm{CDCl}_{3}$
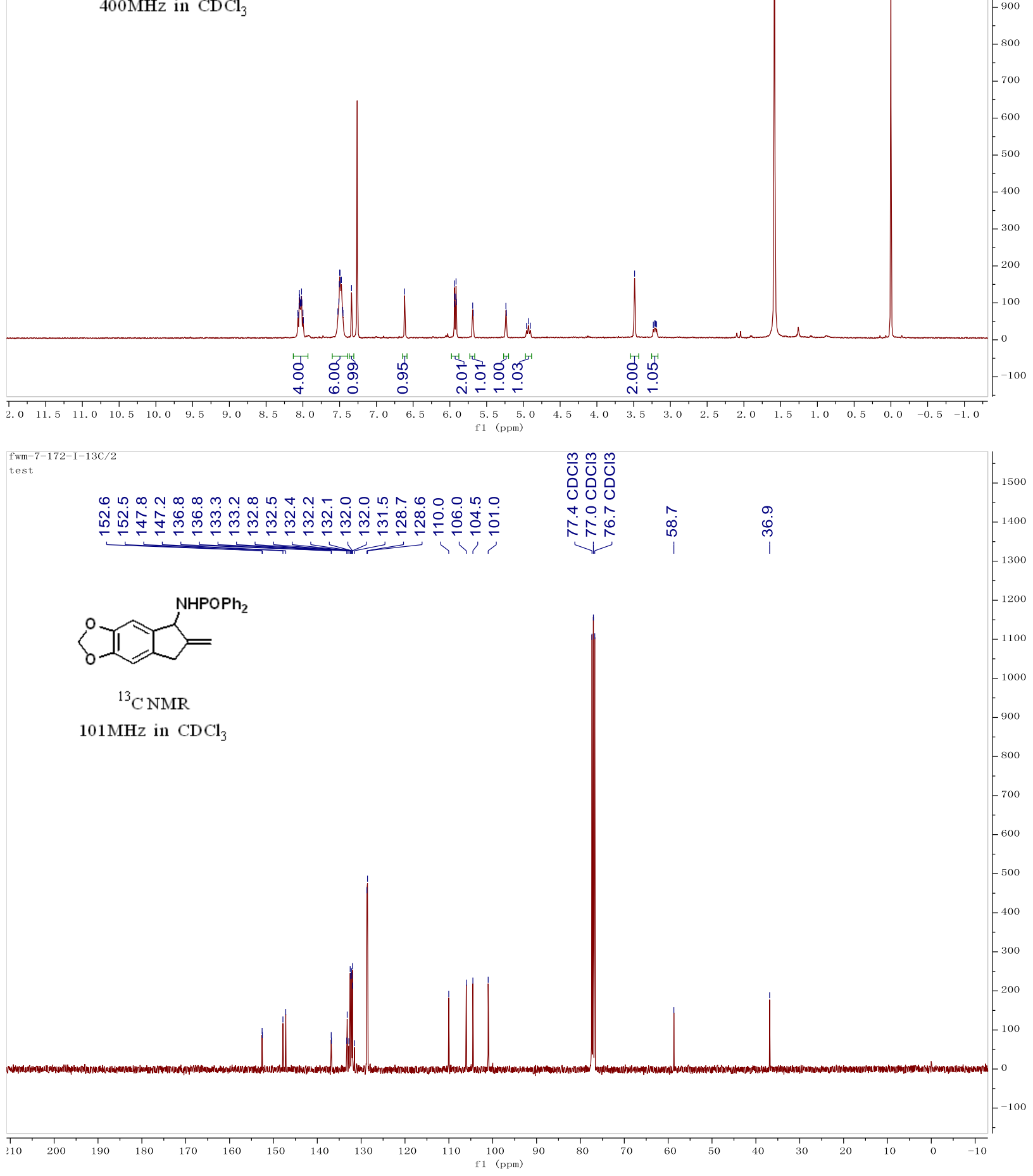


\section{Compound 2q}

我央

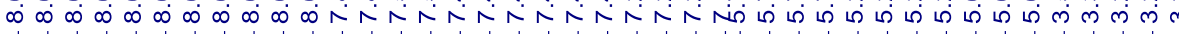
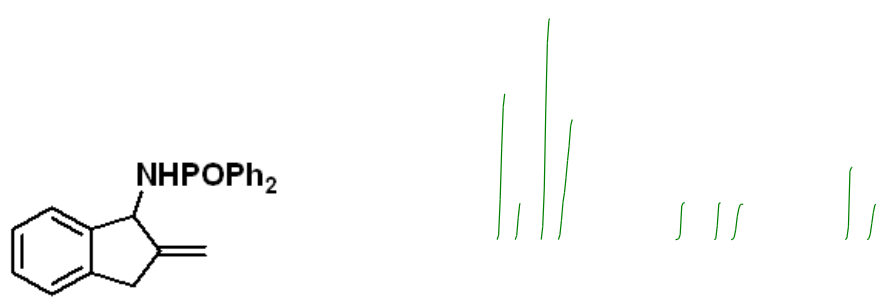

${ }^{1} \mathrm{H}$ NMR

$400 \mathrm{MHz}$ in $\mathrm{CDCl}_{3}$
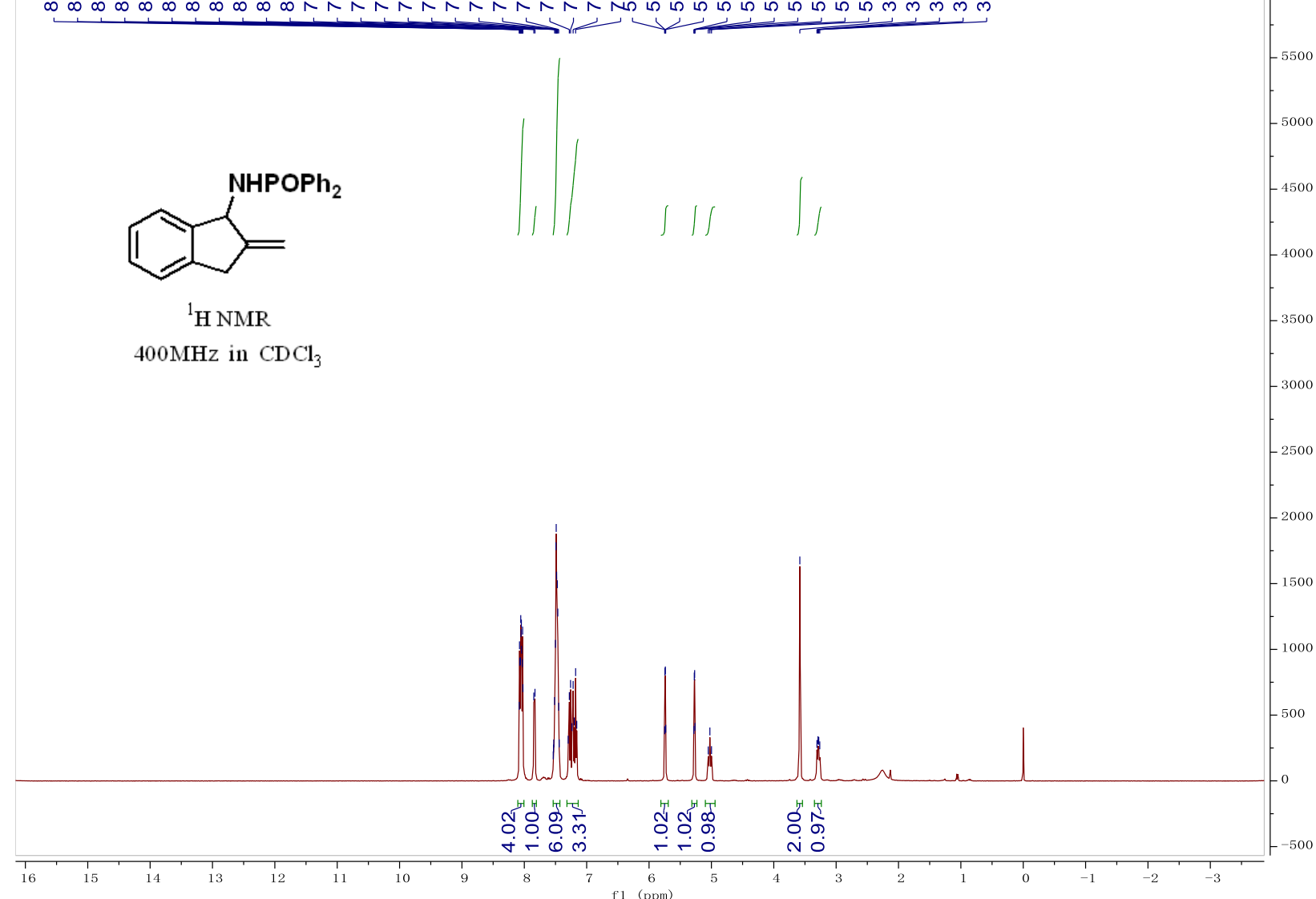
fwm-7-143-recry $/ 2$
C13CPD

ㅂ. 웅 m

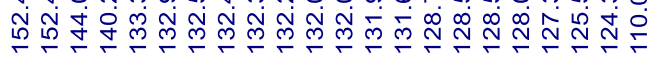

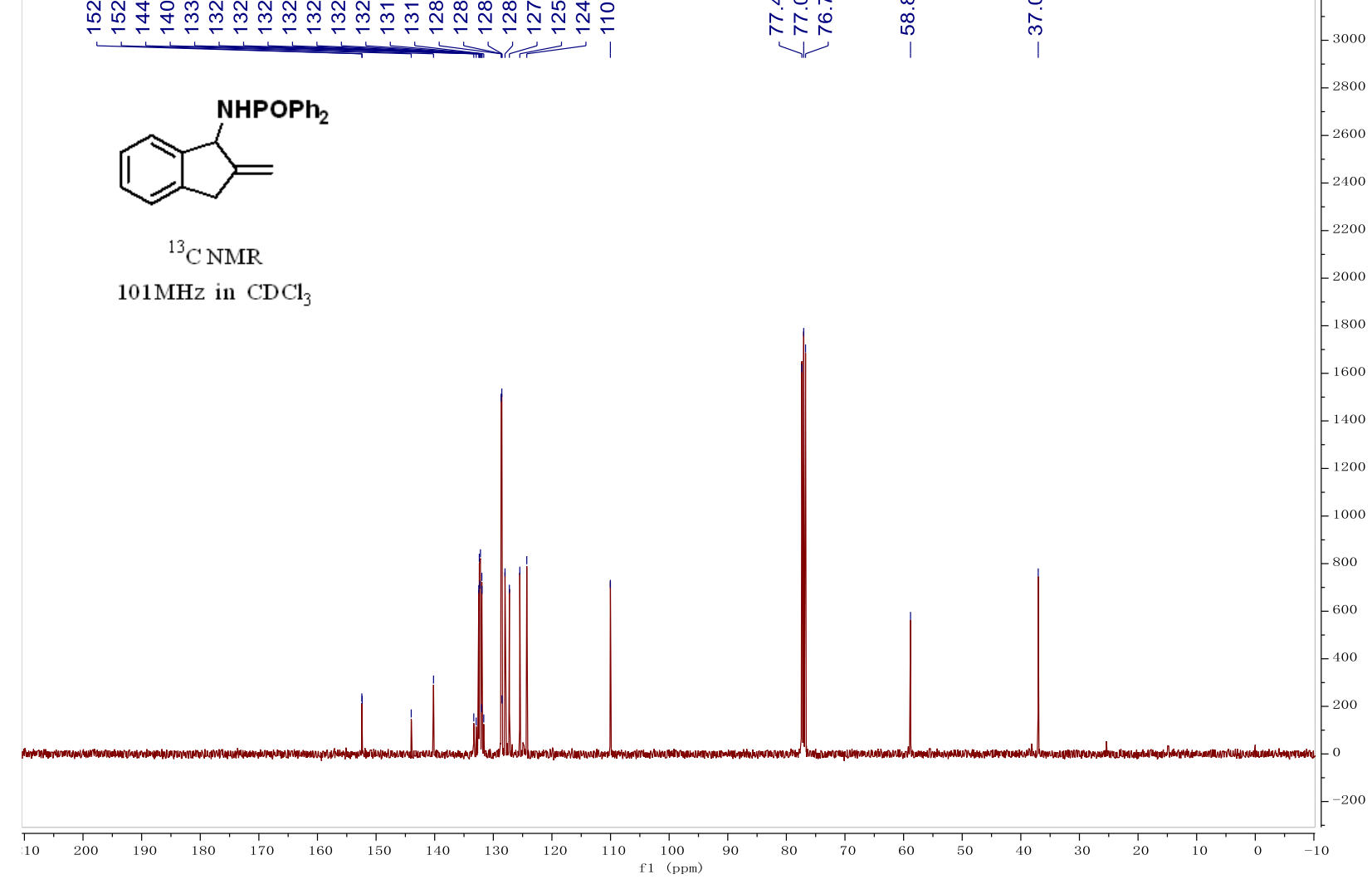




\section{Compound 2s}

MmN 落

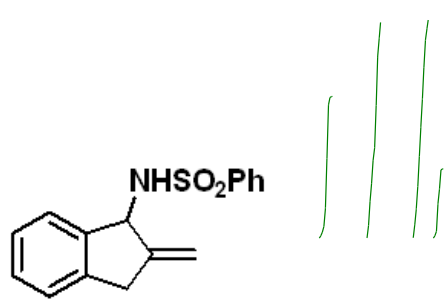

${ }^{1} \mathrm{H}$ NMR

$400 \mathrm{MHz}$ in $\mathrm{CDCl}_{3}$

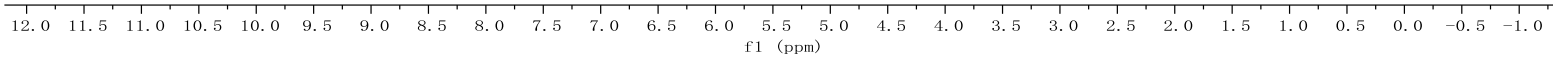
fwm-7-170-recry. 2. fid

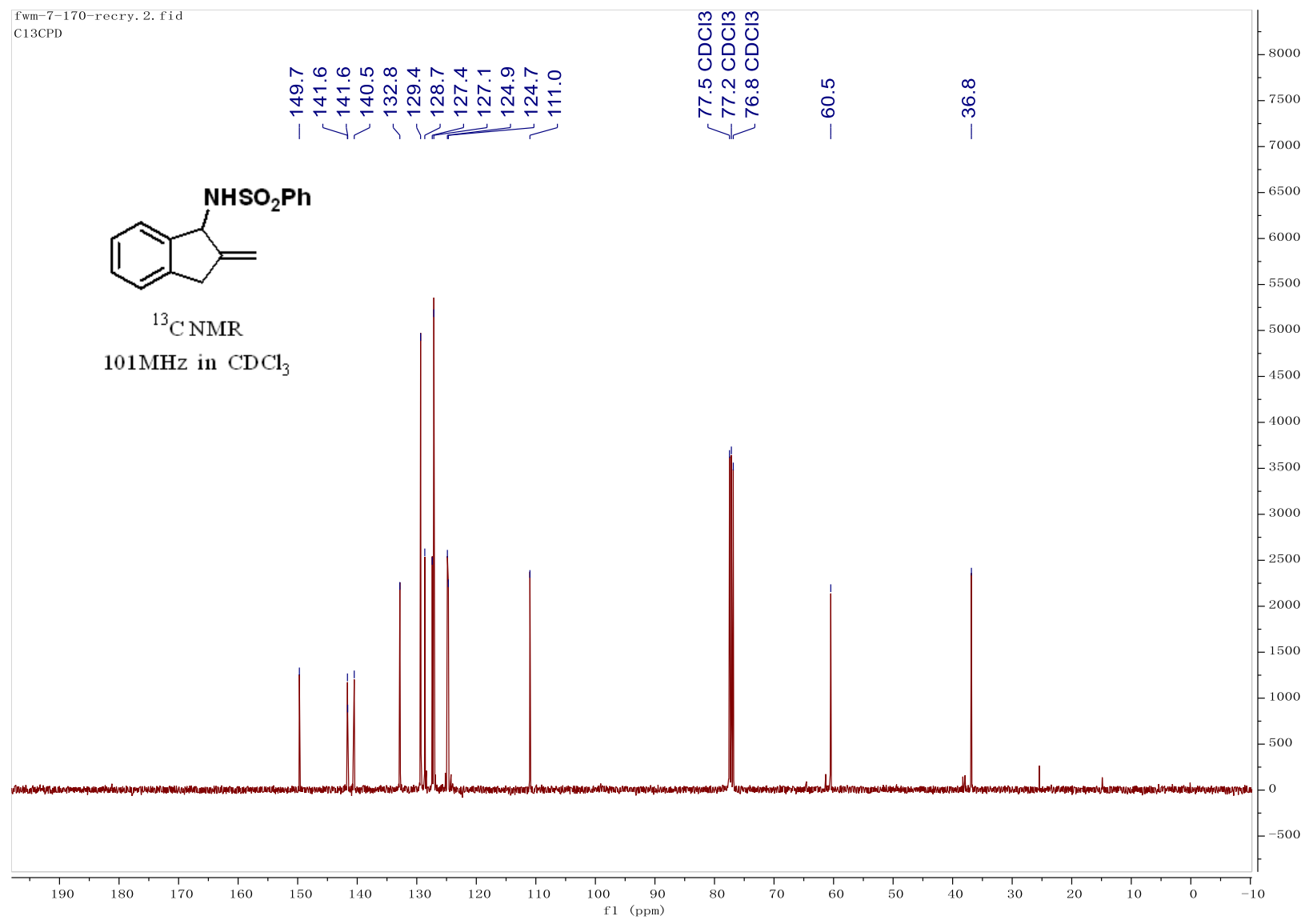




\section{Compound 2r}
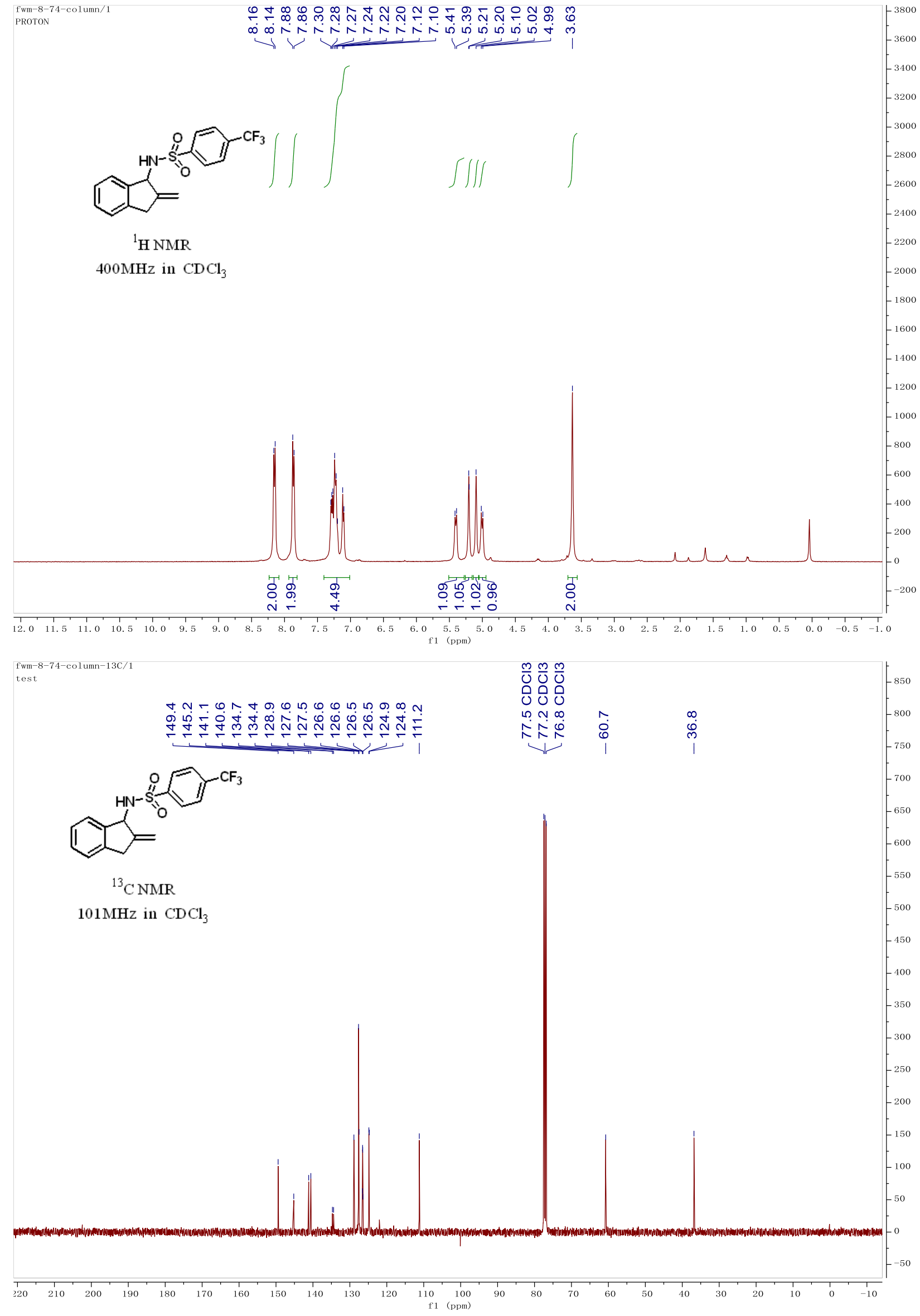


\section{Compound 2t}

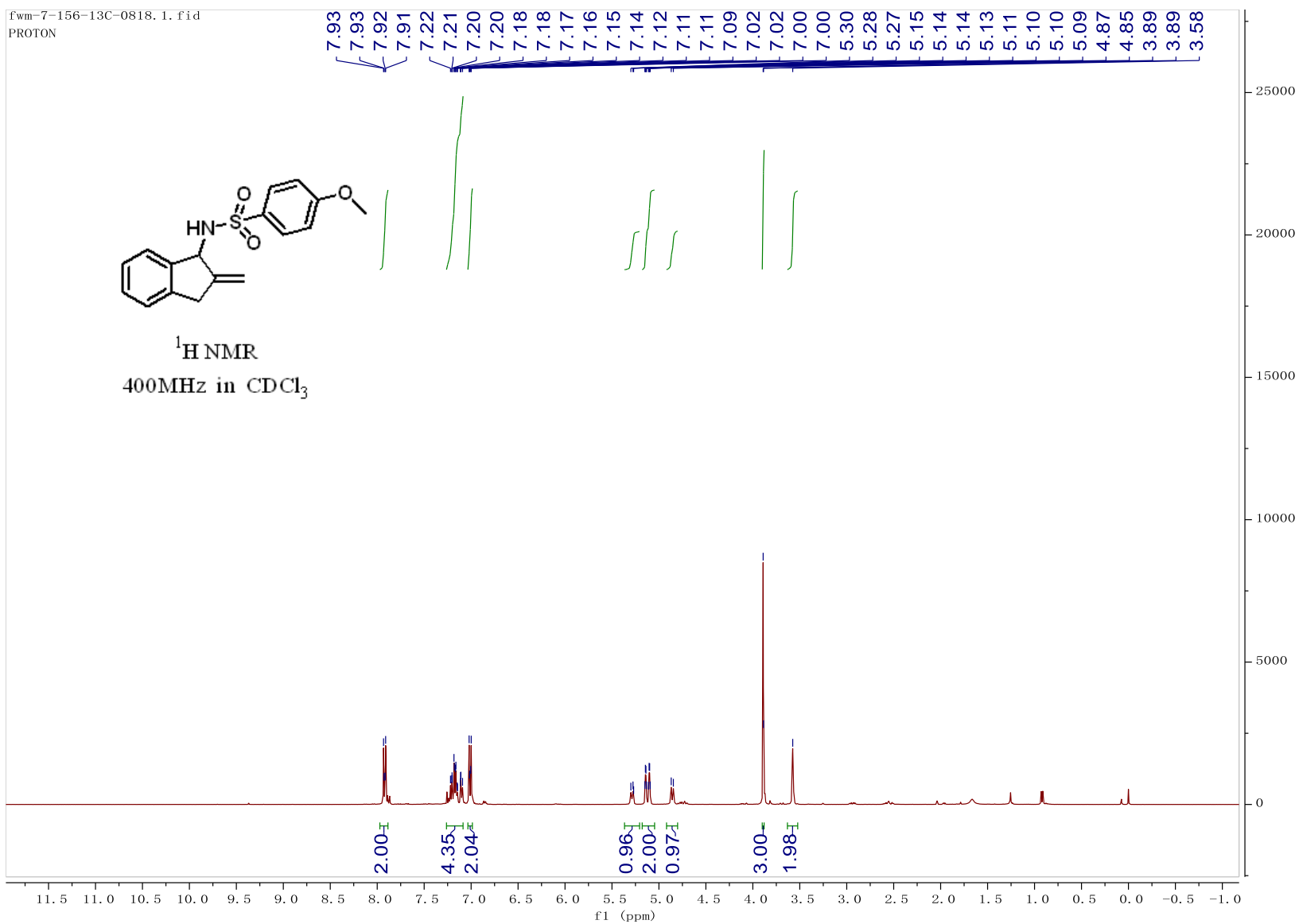

fwm-7-156-13C-0818. 2. fid C13CPD

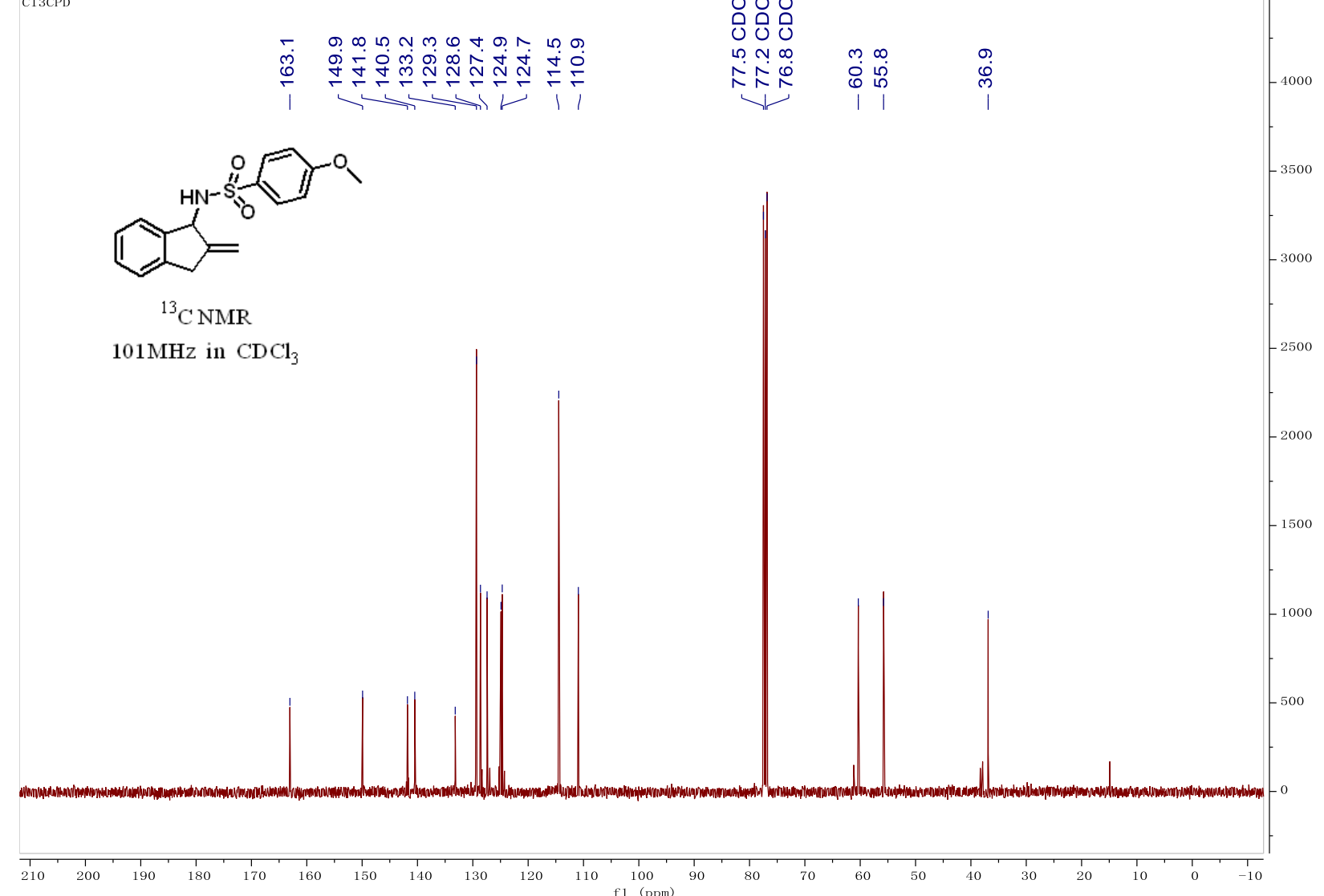




\section{Compound 5a}
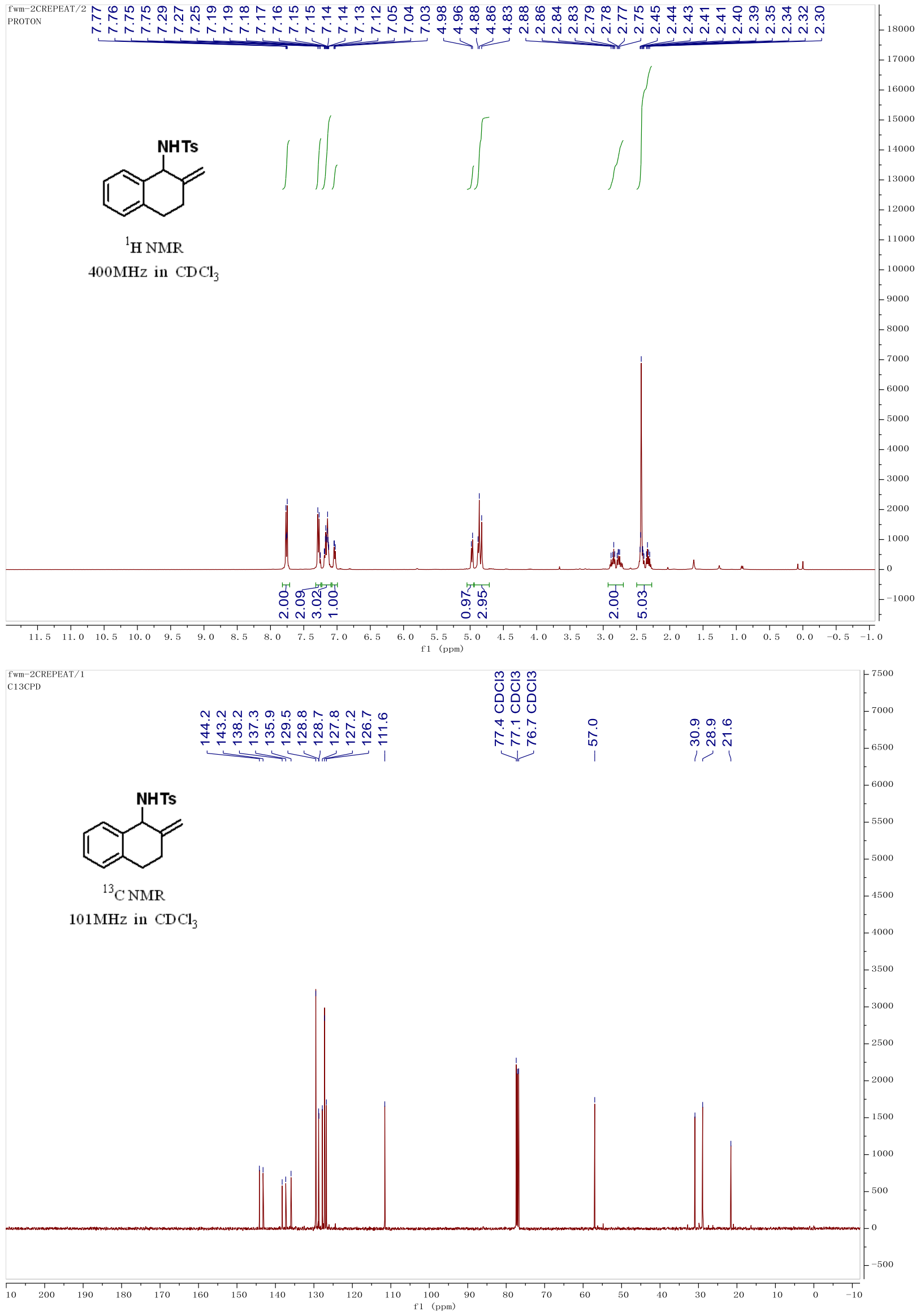


\section{Compound 5b}
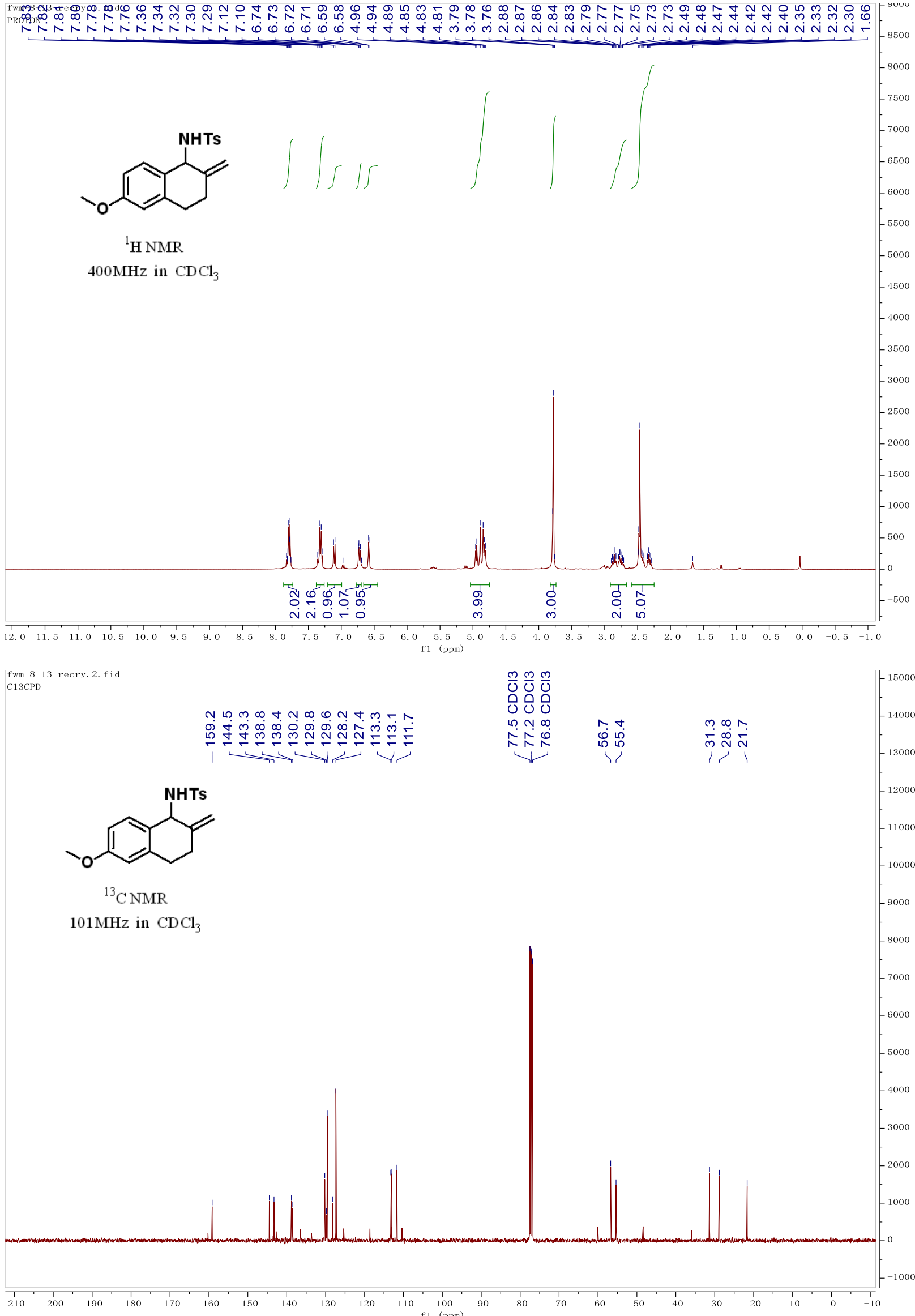


\section{Compound 5c}
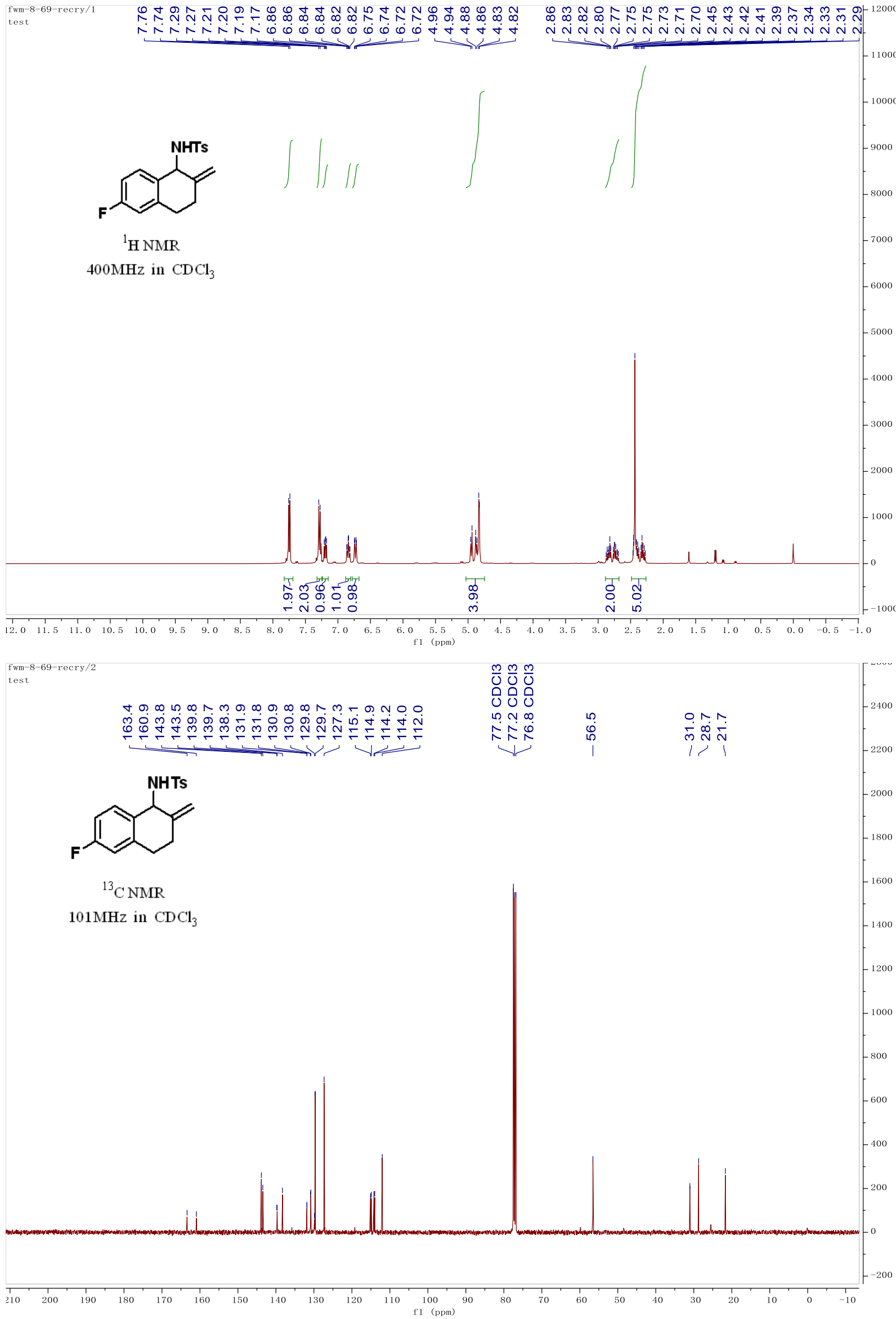


\section{Compound 5d}

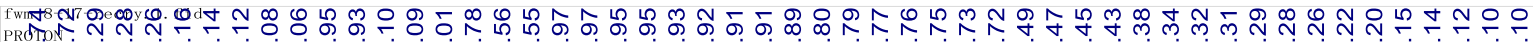

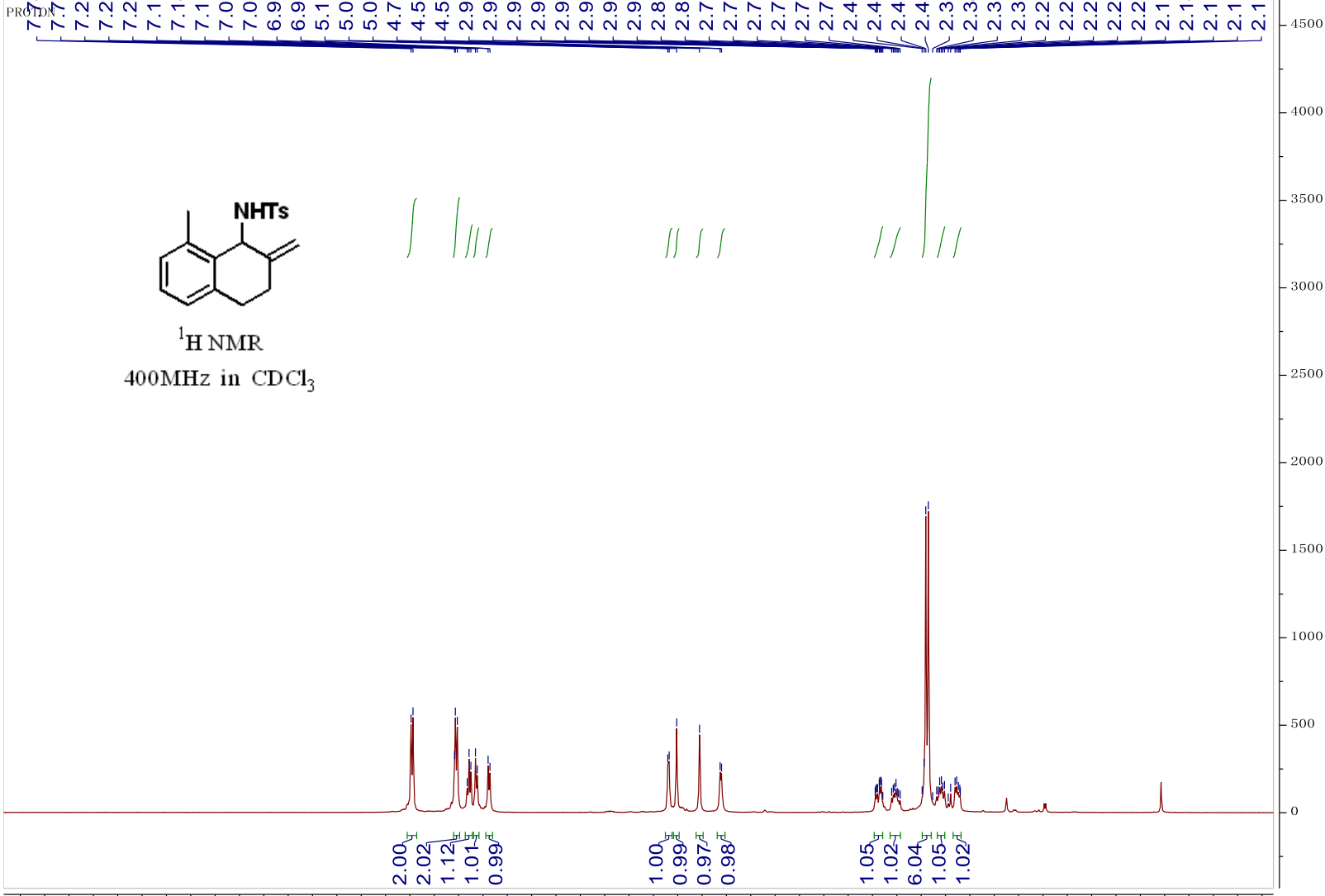

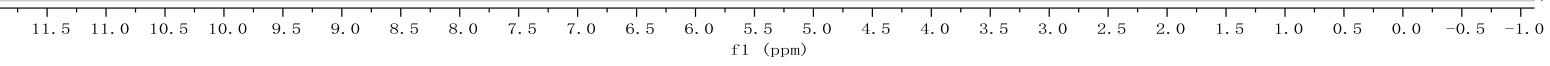

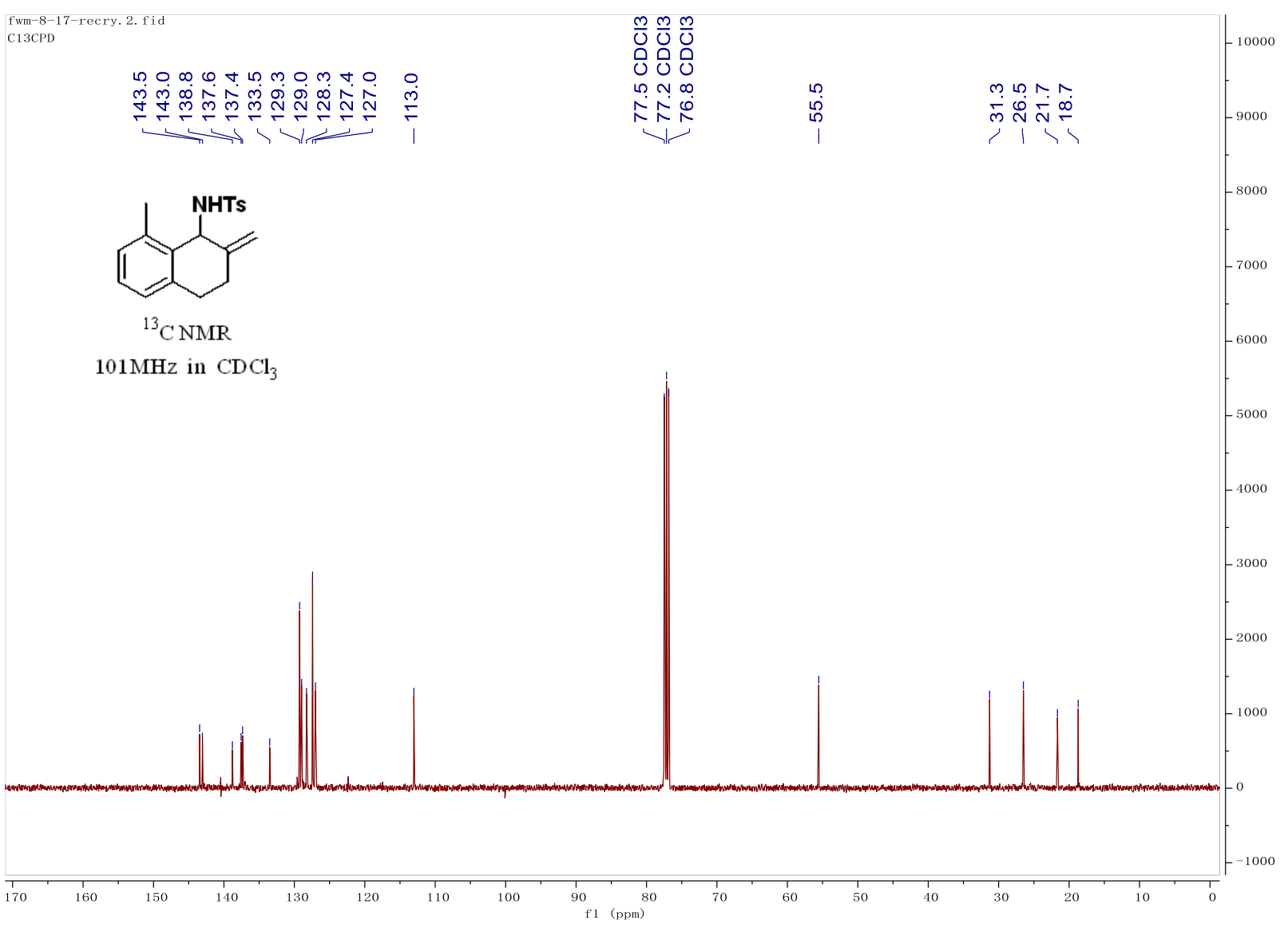




\section{Compound 5e}

fest

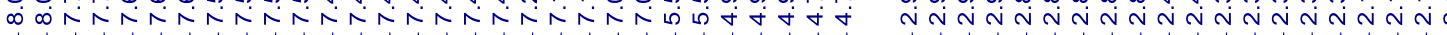

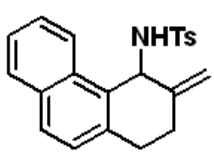

${ }^{1} \mathrm{H}$ NMR

$400 \mathrm{MHz}$ in $\mathrm{CDCl}_{3}$
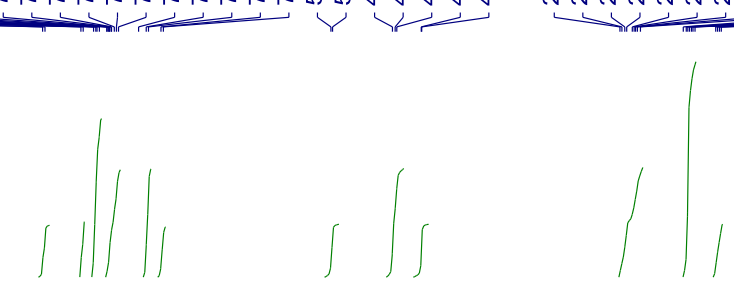

10000

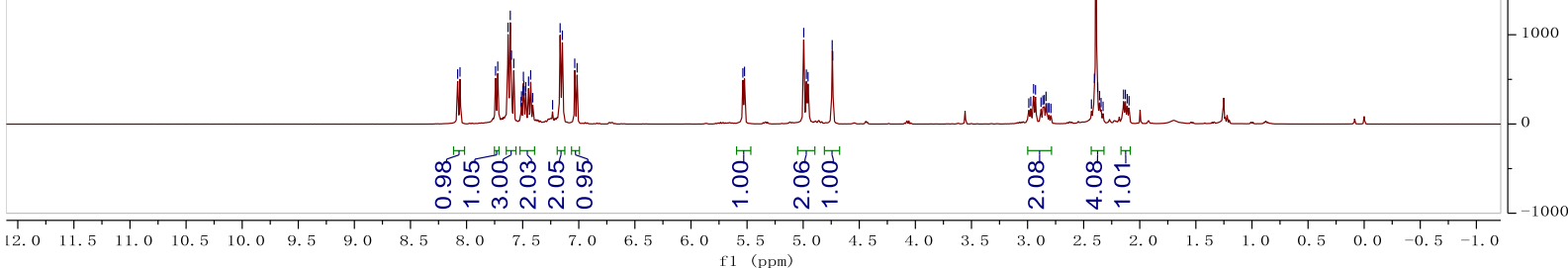

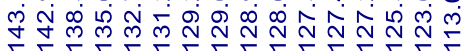

$\frac{m}{0} \frac{m}{0} \frac{m}{0}$

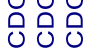

点定点

盛

ìn

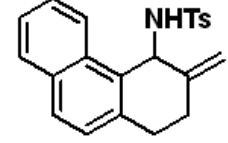

${ }^{13} \mathrm{CNMR}$

$101 \mathrm{MHz}$ in $\mathrm{CDCl}_{3}$

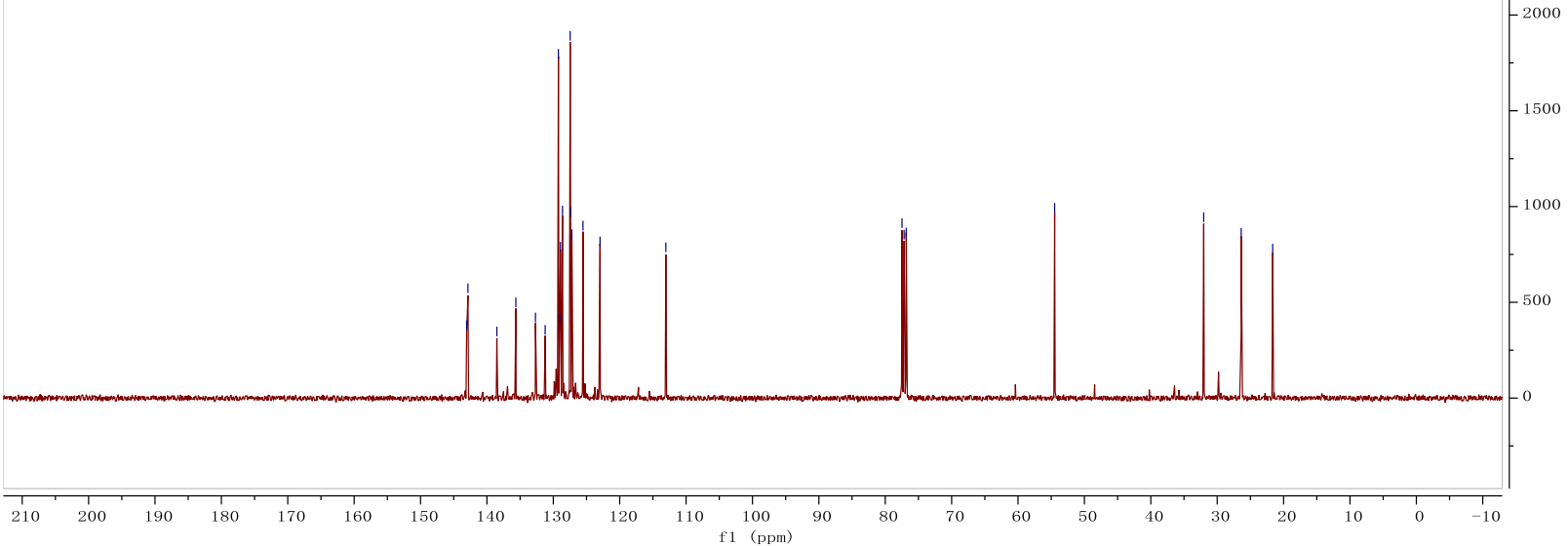




\section{Compound $5 f$}

fwm-8-14-recry. 1. fid PROTON

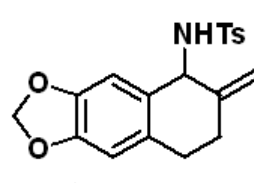

${ }^{1} \mathrm{H} \mathrm{NMR}$

$400 \mathrm{MHz}$ in $\mathrm{CDCl}_{3}$

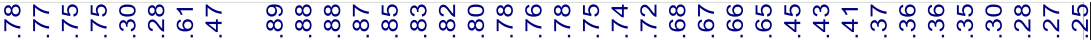

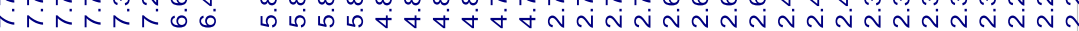

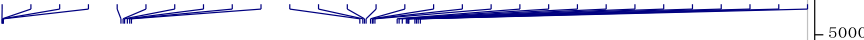

00

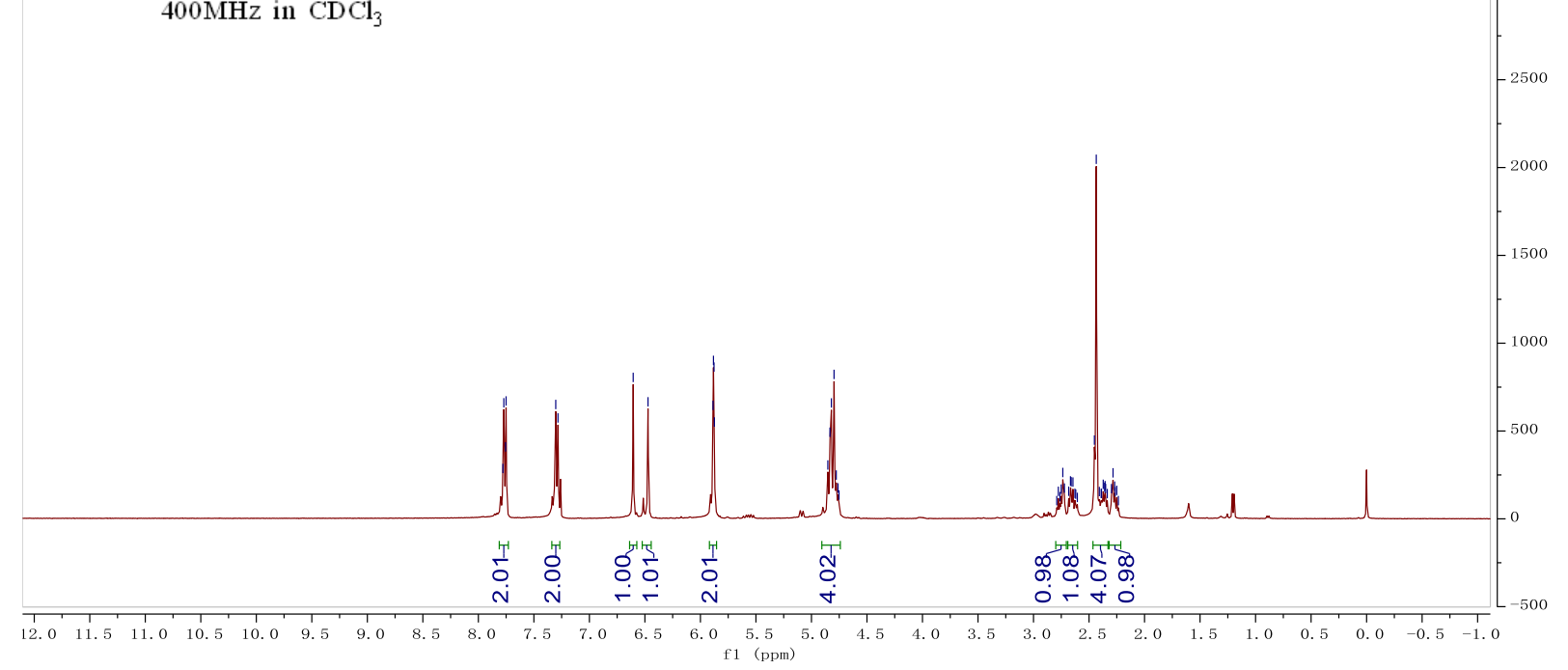
fwm-8-14-recry. 2. fid C13CPD

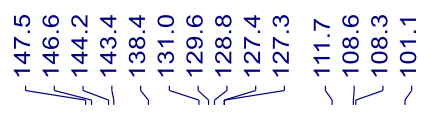

\section{$\frac{m}{0} \frac{m}{0} \frac{m}{0}$}

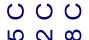

弪 No

है

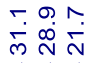

$1 / 1$

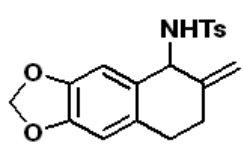

${ }^{13} \mathrm{CNMR}$

$101 \mathrm{MHz}$ in $\mathrm{CDCl}_{3}$

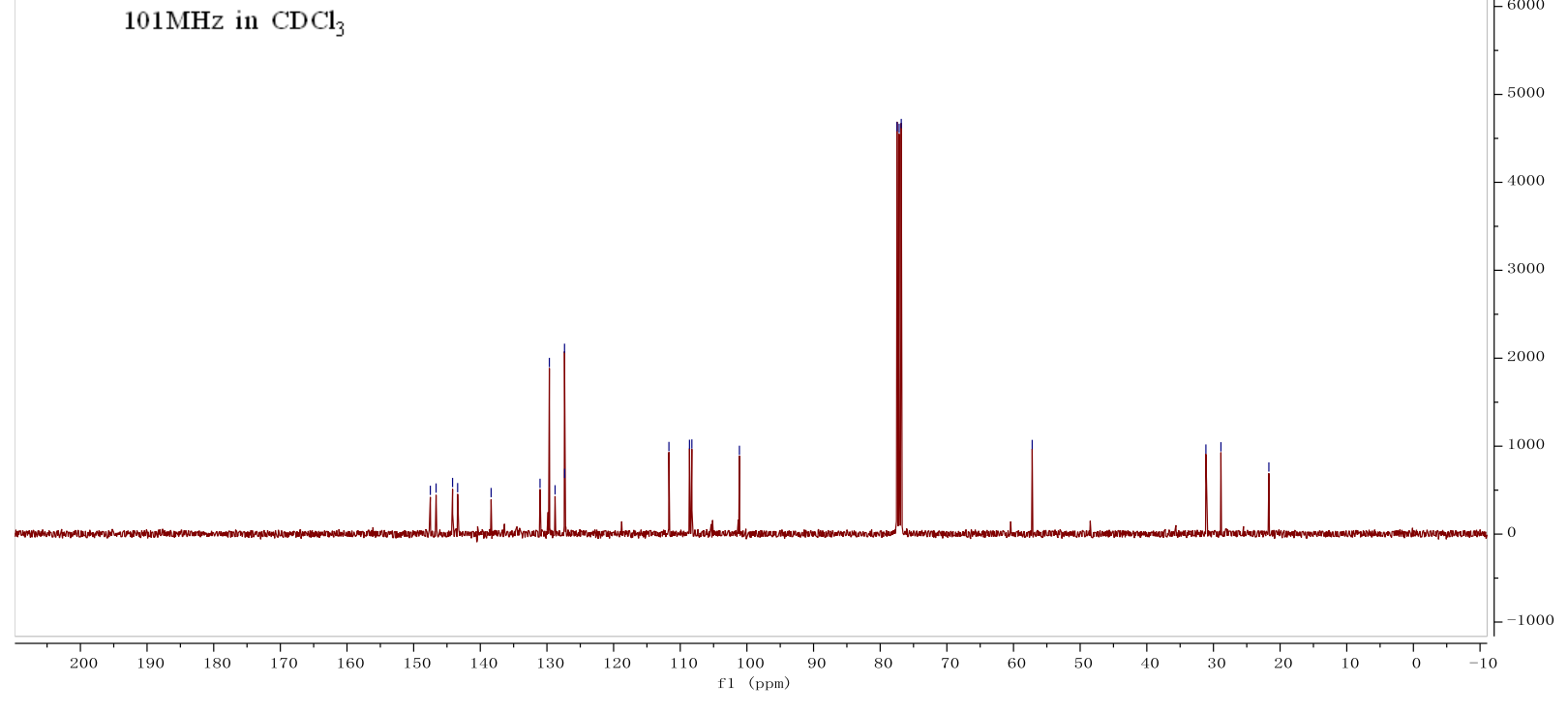




\section{Compound 5g}

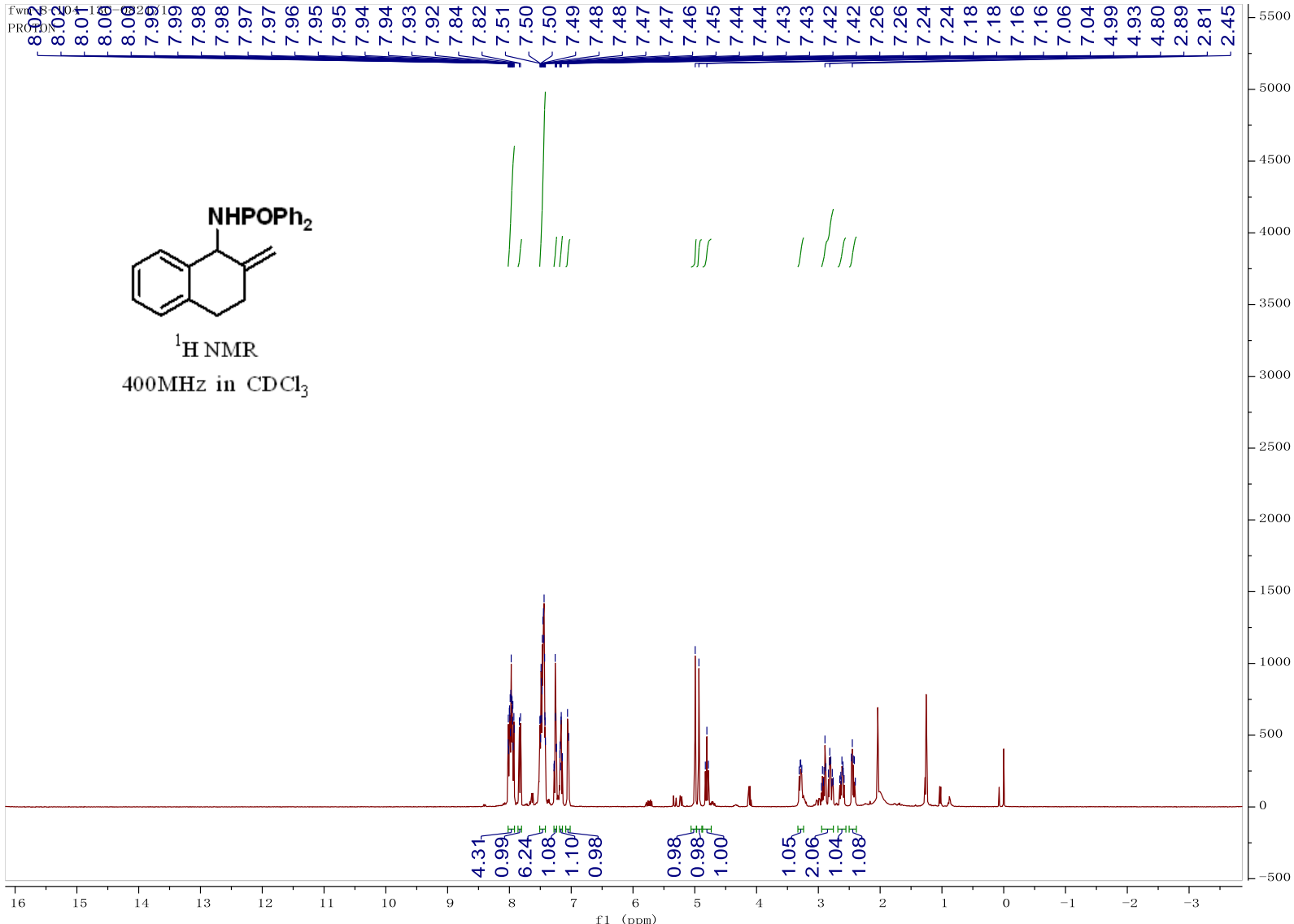

fwm-8-104-13C-0821/2
C13CPD

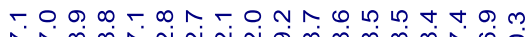

守守究品品

$\frac{m}{0} \frac{m}{0}$
0000
000

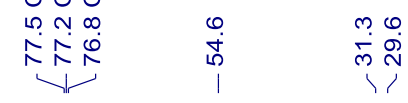

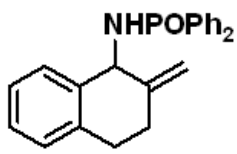

${ }^{13} \mathrm{CNMR}$

$101 \mathrm{MHz}$ in $\mathrm{CDCl}_{3}$

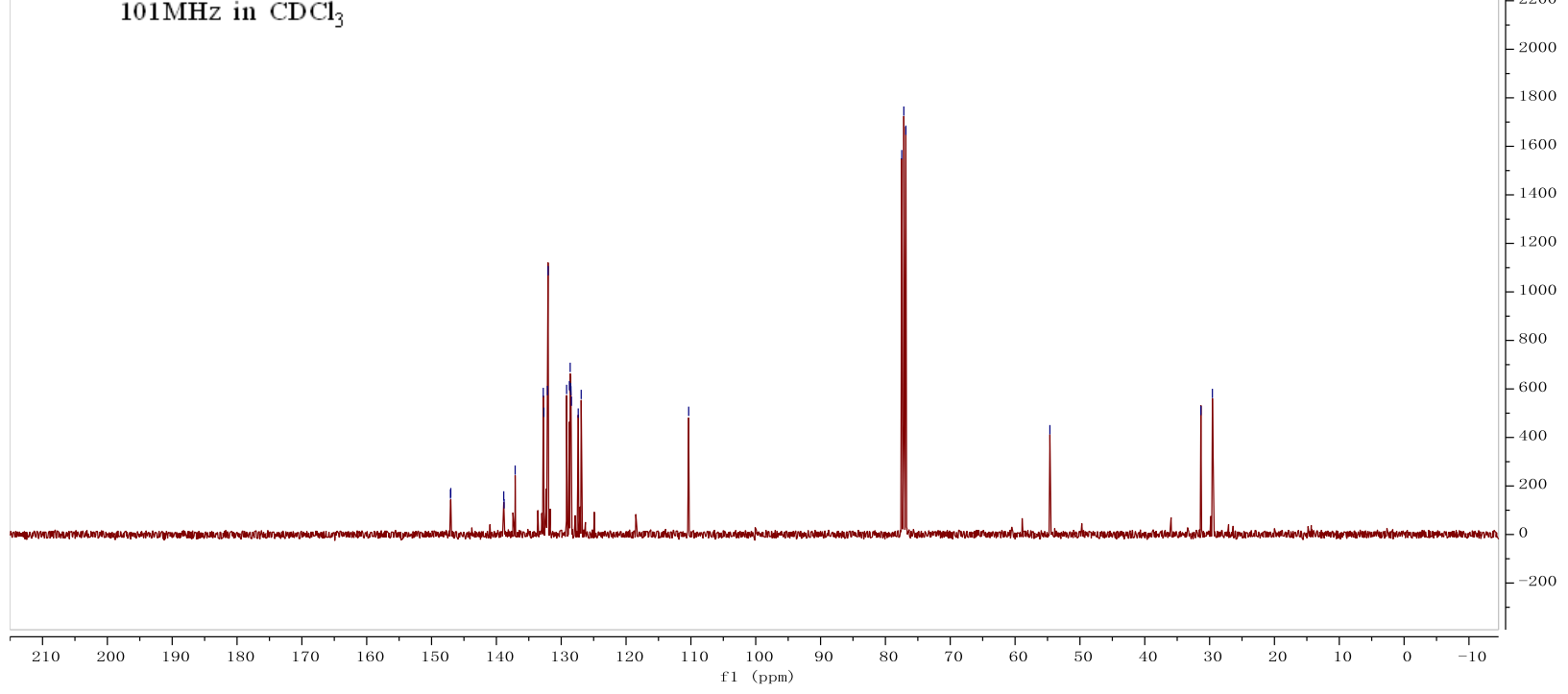




\section{Compound $5 \mathrm{~h}$}
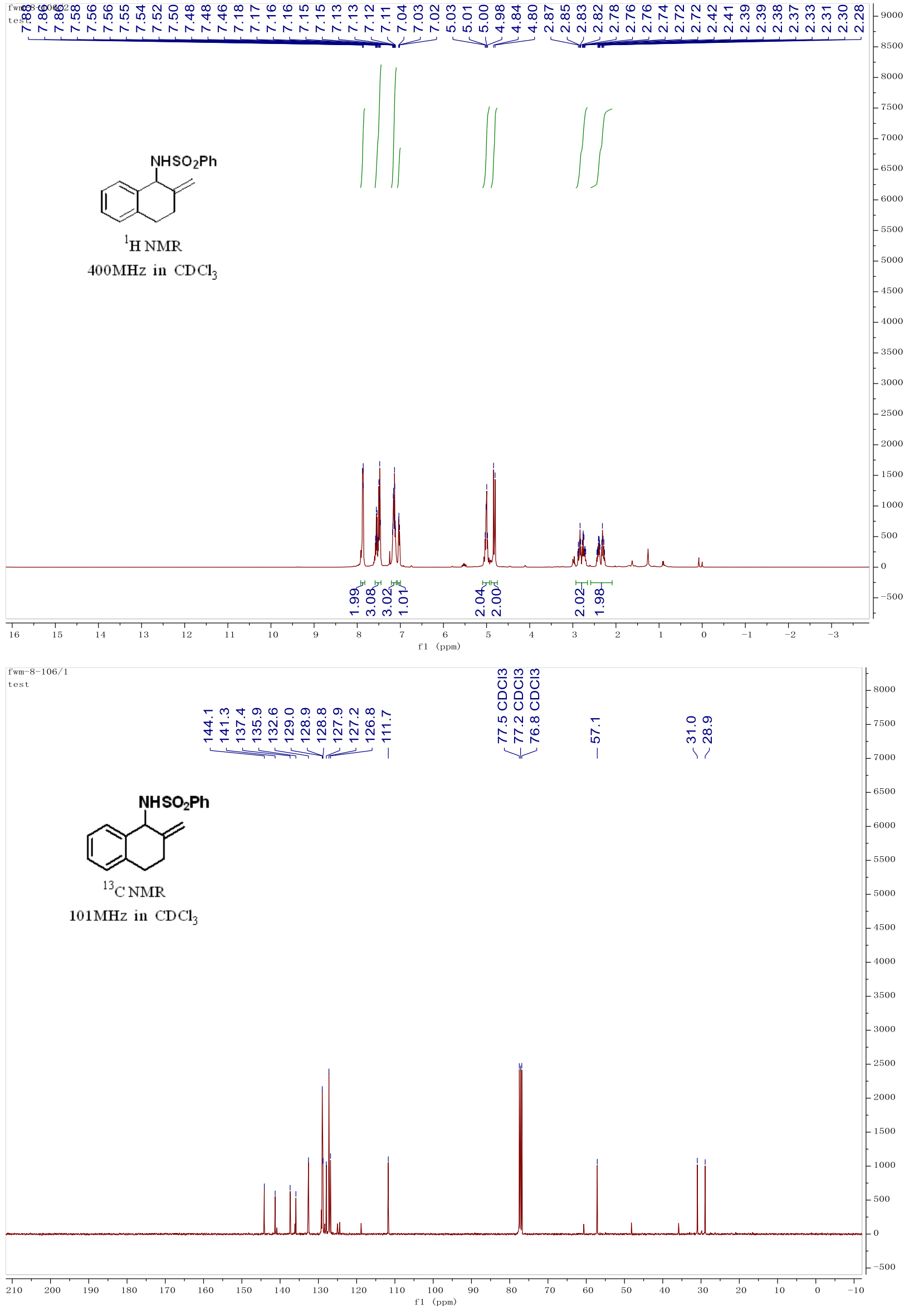


\section{Compound 5i}
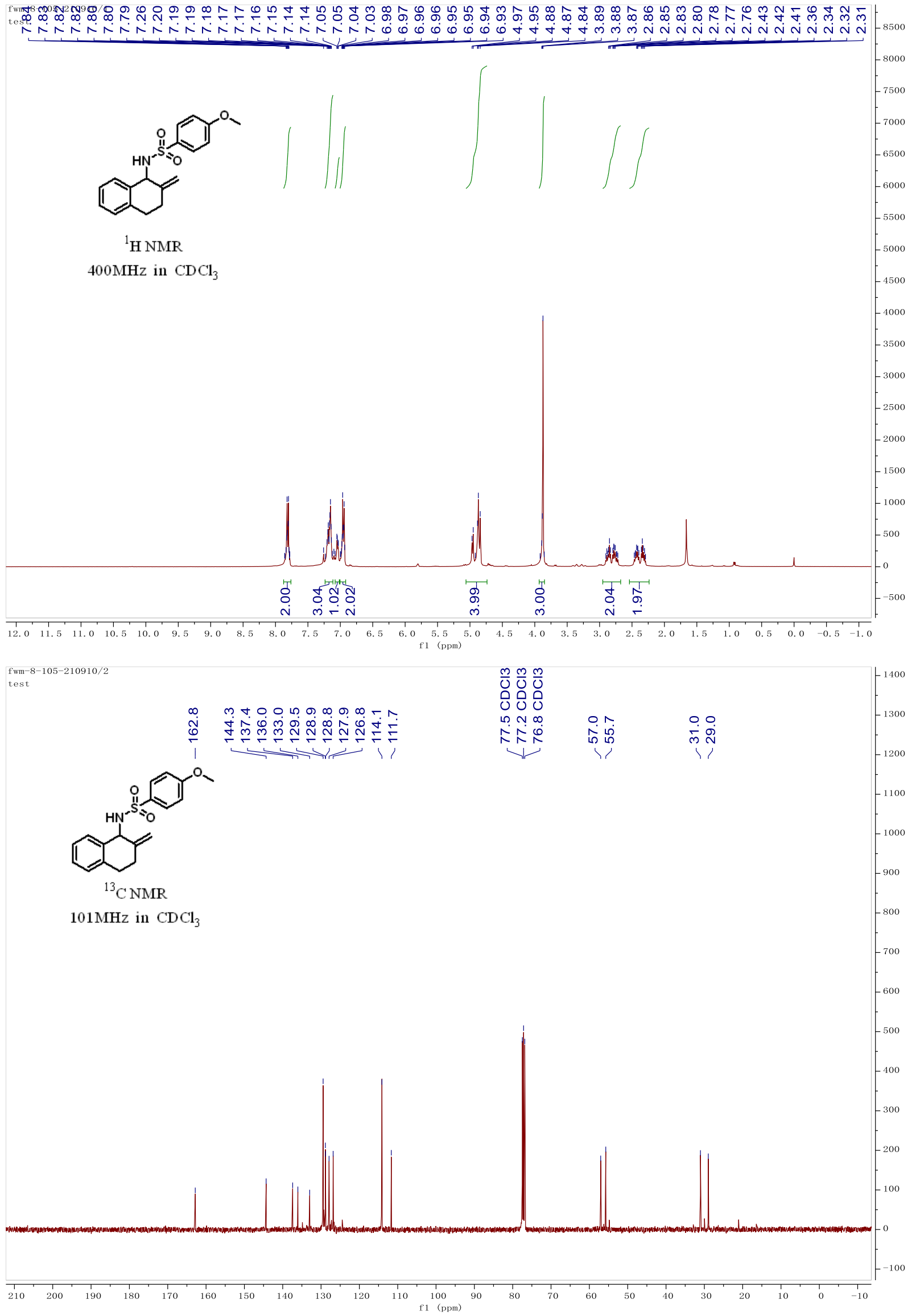


\section{Compound 5j}

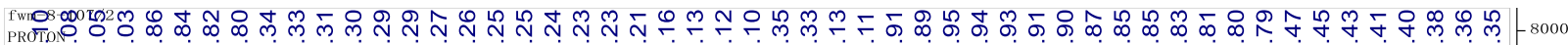

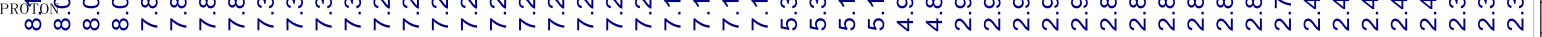
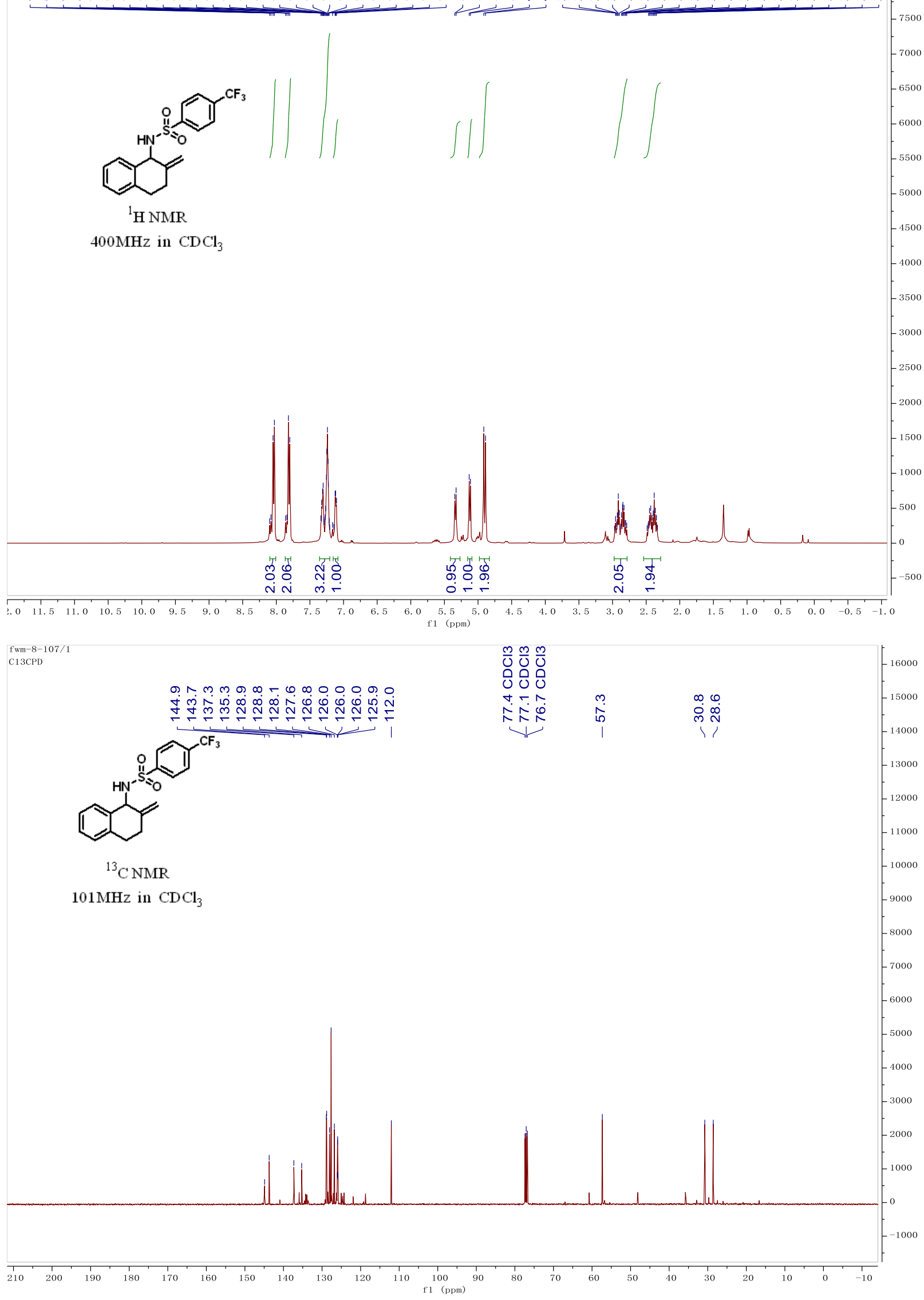


\section{Reference:}

1. Daniels, D. S. B.; Jones, A. S.; Thompson, A. L.; Paton, R. S.; Anderson, E. A. Angew. Chem. Int. Ed. 2014, 53, 1915.

2. Thummanapelli, S. K.; Hosseyni, S.; Su, Y.; Akhmedov, N. G.; Shi, X. Ligand-Controlled Gold-Catalyzed Cycloisomerization of 1,n-Enyne Esters toward Synthesis of Dihydronaphthalene. Chem. Commun. 2016, 52, 7687.

3. Hoshimoto, Y.; Hayashi, Y.; Suzuki, H.; Ohashi, M.; Ogoshi, S. Synthesis of Five- and SixMembered Benzocyclic Ketones through Intramolecular Alkene Hydroacylation Catalyzed by Nickel(0)/N-Heterocyclic Carbenes. Angew. Chem. Int. Ed. 2012, 51, 10812.

4. Watson, I. D. G.; Ritter, S.; Toste, F. D. Asymmetric Synthesis of Medium-Sized Rings by Intramolecular Au(I)-Catalyzed Cyclopropanation. J. Am. Chem. Soc. 2009, 131, 2056.

5. Morales, S.; Guijarro, F. G.; García Ruano, J. L.; Cid, M. B., A General Aminocatalytic Method for the Synthesis of Aldimines. J. Am. Chem. Soc. 2014, 136, 1082. 